\title{
O problema de Nathan Jacobson e questões relacionadas
}

\author{
Victor Hugo López Solís
}

TESE APRESENTADA

$\mathrm{AO}$

Instituto De MATEMÁtica e EstatísticA

DA

Universidade DE SÃo PAulo

PARA

OBTENÇÃO DO TITULO

$\mathrm{DE}$

Doutor EM CIÊNCIAS

Programa: Matemática

Orientador: Prof. Dr. Ivan P. Shestakov

Durante o desenvolvimento deste trabalho o autor recebeu auxilio financeiro da CAPES

São Paulo, Outubro de 2017 


\section{O problema de Nathan Jacobson e questões relacionadas}

Esta versão da tese contém as correções e alterações sugeridas pela Comissão Julgadora durante a defesa da versão original do trabalho, realizada em 30/10/2017. Uma copia da versão original esta disponível no Instituto de Matemática e Estatística da Universidade de São Paulo.

Comissão Julgadora:

- Prof. Dr. Ivan Shestakov (orientador) - IME-USP

- Prof. Dr. Lucia Satie Ikemoto Murakami - IME-USP

- Prof. Dr. Victor Petrogradskiy - UnB

- Prof. Dr. Alexandr Kornev - UFABC

- Prof. Dr. Faber Alberto Gómez González - Universidade de Antioquia 


\section{Agradecimentos}

Em primeiro lugar, quero agradecer a Cristo nosso Senhor por guiar meus passos, por ter me fortalecido em momentos de angústia, desespero e também de alegria, e também por ter permitido cumprir um dos meus grandes desejos, obter o doutorado.

Eu também quero agradecer a muitas pessoas que me ajudaram a cumprir este sonho. Meus mais sinceros agradecimentos ao professor Ivan Shestakov, pela sua sabedoria e paciência em me guiar, pois as inúmeras conversas e suas palavras foram fundamentais na minha formação como matemático.

Agradeço também a minha família, a minha mãe Eugenia Hermelinda, ao meu pai Victor Modesto e ao meu irmão Jesús Miguel por me dar amor, carinho e que apesar da distância conseguiram acompanhar-me nos meus dias de luta para tornar este sonho realidade. Essa conquista é para vocês.

Agradeço aos meus amigos do CRUSP, onde vivi momentos tristes e felizes, e onde conheci muitas pessoas que me ajudaram a ser feliz todos os dias. Meus agradecimentos especiais são para minha amiga Janeth Marlene e para meu irmão Johnny César, a companhia de ambos me fez sentir e ver que eu não estava sozinho. Muito obrigado a ambos pelo amor, companhia e amizade, e pelo que compartilhamos; refeições, risos, jogos e conversas ... coisas que fazem a vida valer a pena.

Como não lembrar dos meus amigos que me acompanharam todos esses anos no IME, aqui é um momento importante para agradecer de forma especial a sua amizade e companhia; a Diego (Chupito), Carlos (Charly), Daniel (Danielito) e Pablo (Pablito). Obrigado pelos risos e conversas que fizeram sentir me em boa companhia.

Agradeço ao IME (Instituto de Matemática e Estatística) por todo o apoio, especialmente aos professores e administrativos, por me oferecer um bom ambiente de trabalho. 


\section{Resumo}

López Solís V. H. O problema de Nathan Jacobson e questões relacionadas. 2017. 120 f. Tese (Doutorado) - Instituto de Matemática e Estatística, Universidade de São Paulo, São Paulo, 2017.

Este trabalho consiste de três partes: "Teoremas de coordenatização de Wedderburn e de Zorn", "O problema de Nathan Jacobson" e "Teoremas de Fatorização de Kronecker para as superálgebras alternativas".

$\mathrm{Na}$ primeira parte apresentamos os teoremas de coordenatização de Wedderburn e de Zorn e suas aplicações na teoria de representações das álgebras associativas e alternativas.

Na segunda parte resolvemos um problema de longa data que foi anunciado por Nathan Jacobson sobre a descrição das álgebras alternativas que contém $M_{2}(F)$ (álgebra associativa de matrizes $2 \times 2$ ) com o mesmo elemento identidade.

$\mathrm{Na}$ terceira parte damos uma prova independente que é válida em qualquer característica do clássico Teorema de Fatorização de Kronecker de Nathan Jacobson. Generalizamos esse resultado e provamos um teorema de Fatorização de Kronecker para as superálgebras alternativas cuja parte par contém $\mathbb{O}$ com o mesmo elemento identidade. Além disso, provamos um Teorema de Fatorização de Kronecker para as superálgebras alternativas que contêm a superálgebra associativa $M_{(1 \mid 1)}(F)$ com o mesmo elemento identidade. Como Corolário desse resultado, respondemos a um análogo do problema de Jacobson para as superálgebras alternativas, isto é, descrevemos as superálgebras alternativas que contêm à superálgebra associativa $M_{(1 \mid 1)}(F)$ com o mesmo elemento identidade. Finalmente, estudamos as representações das superálgebras alternativa simples $\mathbb{O}(4 \mid 4)$ e $\mathbb{O}[u]$. Classificamos os bimodules sobre essas superálgebras e provamos alguns análogos do Teorema de Fatorização de Kronecker para as superálgebras alternativas que contenham $\mathbb{O}(4 \mid 4)$ ou $\mathbb{O}[u]$ com o mesmo elemento identidade.

Palavras-chave: (Super) álgebras alternativas, teoremas de coordenatização, teoremas de fatorização de Kronecker. 


\section{Abstract}

López Solís V. H. The Nathan Jacobson's problem and related questions. 2017. 120 f. Tese (Doutorado) - Instituto de Matemática e Estatística, Universidade de São Paulo, São Paulo, 2017.

This work consists of three parts: "Wedderburn and Zorn coordinatizations theorems", "Nathan Jacobson's problem" and "Kronecker's Factorization theorems for alternative superalgebras".

In the first part we present Wedderburn and Zorn coordinatizations theorems and their applications in the theory of representations of associative and alternative algebras.

In the second part we solve a long standing problem that was announced by Nathan Jacobson on the description of alternative algebras containing $M_{2}(F)$ (associative matrix algebra $2 \times 2$ ) with the same identity element.

In the third part we give an independent proof that is valid in any characteristic of Nathan Jacobson's classic Kronecker Factorization Theorem. We generalize this result and prove a Kronecker Factorization Theorem for alternative superalgebras whose even part contains $\mathbb{O}$ with the same identity element. In addition, we prove a Kronecker Factorization Theorem for alternative superalgebras containing the associative superalgebra $M_{(1 \mid 1)}(F)$ with the same identity element. As a corollary of this result, we respond to an analogue of Jacobson's problem for alternative superalgebras, that is, we describe the alternative superalgebras containing the associative superalgebra $M_{(1 \mid 1)}(F)$ with the same identity element. Finally, we study the representations of the simple alternative superalgebras $\mathbb{O}(4 \mid 4)$ e $\mathbb{O}[u]$. We classify the bimodules on these superalgebras and prove some analogues of the Kronecker Factorization Theorem for alternative superalgebras containing $\mathbb{O}(4 \mid 4)$ or $\mathbb{O}[u]$ with the same identity element .

Keywords: Alternative algebras, coordinatization theorems, Kronecker's factorization theorems. 


\section{Sumário}

$\begin{array}{ll}\text { Introdução } & 1\end{array}$

1 Algumas (super)álgebras não associativas $\quad 5$

1.1 Álgebras e álgebras livres . . . . . . . . . . . . . . . . . . . . 5 5

1.2 Álgebras livres em uma variedade . . . . . . . . . . . . . . . . . 6

1.3 Bimódulos . . . . . . . . . . . . . . . . . . . . . . 7

1.4 Superálgebras . . . . . . . . . . . . . . . . . . . . . . . 9 9

2 Teoremas de Coordenatização de Wedderburn e de Zorn 11

2.1 O Teorema de coordenatização de Wedderburn . . . . . . . . . . . . . . . . 11

2.2 O Teorema de coordenatização de Zorn . . . . . . . . . . . . . . . . . . . . . 14

2.3 Generalização de um teorema de Nathan Jacobson . . . . . . . . . . . . . . . 25

3 O problema de Nathan Jacobson $\quad 31$

3.1 Álgebras e bimódulos alternativos . . . . . . . . . . . . . . . . . 32

3.1 .1 Álgebras alternativas . . . . . . . . . . . . . . . . . . 32

3.1.2 Bimódulos alternativos . . . . . . . . . . . . . . . . 33

3.2 Formulação do problema . . . . . . . . . . . . . . . . . . . . . 34

3.3 Solução do problema . . . . . . . . . . . . . . . . . . . . . 35

3.3.1 Álgebras alternativas tensoriais . . . . . . . . . . . . . . 49

3.4 Respostas do problema de Nathan Jacobson . . . . . . . . . . . . . . . . . 60

3.5 Álgebras e bimódulos alternativos com involução . . . . . . . . . . . . . . . . 61

4 Teoremas de Fatorização de Kronecker para as superálgebras alternativas 65

4.1 Superálgebras e (super) bimódulos alternativos . . . . . . . . . . . . . 66

4.1.1 Superálgebras alternativas . . . . . . . . . . . . . . . 66

4.1 .2 Superimódulos alternativos . . . . . . . . . . . . . . 67

4.2 Um análogo do problema de Nathan Jacobson para as superálgebras alternativas 68

4.3 Teoremas de Fatorização de Kronecker . . . . . . . . . . . . . . . . . . . . 69

$\begin{array}{ll}\text { Referências Bibliográficas } & 83\end{array}$ 


\section{Introdução}

"Só sei que nada sei." (Sócrates)

As álgebras alternativas são álgebras não-associativas e nem comutativas que satisfazem as versões debilitadas de associatividade

$$
(x y) y=x y^{2}, \quad x^{2} y=x(x y) .
$$

É claro que toda álgebra associativa, é alternativa. Um exemplo clássico de uma álgebra alternativa não-associativa, é a álgebra de octônios $\mathbb{O}$. Esta álgebra foi descoberta antes da álgebra de matrizes, mas a teoria sistemática das álgebras alternativas teve ainda seu começo nos anos 30, nos trabalhos de Zorn e Moufang dedicados a planos projetivos.

Assim, desde aquele tempo, as álgebras alternativas, junto com as álgebras de Jordan, encontraram aplicações fundamentais em várias partes da matemática e na física e agora formam uma parte intrínseca de métodos algébricos modernos.

A teoria estrutural das álgebras alternativas está bem desenvolvida [1, 6, 12, 19]. Essa teoria tem as mesmas características da teoria das álgebras associativas. Por exemplo, cada álgebra alternativa contém um radical (ideal maximal quase-regular) tal que o quociente por este ideal é soma subdireta de álgebras primitivas. Daí, o famoso teorema de Kleinfeld diz que cada álgebra alternativa simples (ou primitiva) é associativa ou isomorfa à álgebra de octônios.

Nas teorias das álgebras associativas, alternativas e de Jordan, os chamados teoremas de coordenatização desempenham um papel importante. Um teorema clássico deste tipo é o teorema de coordenatização de Wedderburn para as álgebras associativas, ele diz que se uma álgebra associativa unitária $\mathcal{A}$ contém uma subálgebra $M_{n}(F)$ de matrizes $n \times n$ como o mesmo elemento identidade, então $\mathcal{A} \cong \mathcal{B} \otimes_{F} M_{n}(F)$ para uma certa subálgebra $\mathcal{B}$ de $\mathcal{A}$. Kaplansky no Teorema 2 de [4] estendeu o resultado de Wedderburn para as álgebras alternativas $\mathcal{A}$ e $\mathcal{B}$ a álgebra de Cayley. Em um dos seus trabalhos, Nathan Jacobson deu uma nova prova do resultado de Kaplansky, usando sua classificação da redutibilidade completa dos bimódulos alternativos e provou um análogo desse teorema para as álgebras de Jordan [2], onde o papel da álgebra de Cayley é desempenhado pela álgebra de Jordan simples excepcional de dimensão 27 de Albert. Estes resultados tem aplicações importantes na teoria de representações de álgebras alternativas e de Jordan [1, 3].

Nossos principais objetivos são:

- Descrever o Teorema de coordenatização de Wedderburn e aplica-lo na teoria de representação de álgebras associativas. Provar o Teorema de coordenatização de Zorn que foi deixado como um exercício em [19] e aplica-lo na teoria de representação de álgebras alternativas.

- Resolver o problema de Nathan Jacobson que foi anunciado em [2]. 
- Oferecer uma demonstração que funcione para o caso geral do clássico Teorema de Fatorização de Kronecker de Jacobson. Generalizar esse resultado para as superálgebras alternativas. Resolver um análogo do problema de Jacobson para as superálgebras alternativas. Finalmente, obter um Teorema de Fatorização de Kronecker para as superálgebras alternativas que contêm as superálgebras alternativas simples com o mesmo elemento identidade.

Assim, nosso trabalho começa com:

O capítulo 1 que é dedicado as diferentes classes de (super)álgebras. Nesse capítulo incluimos algumas definições, exemplos de classes de (super)álgebras que usamos nos seguintes capítulos. O capítulo foi embasada nos livros de E. N. Kuzmin e I. Shestakov Nonassociative structures [6], R. D. Schafer An Introduction to Nonassociative Algebras [12] e o artigo de I. Shestakov Alternative and Jordan superalgebras [15].

O capítulo 2 refere-se a dois teoremas de coordenatização. Na seção 2.1, apresentamos o Teorema de coordenatização de Wedderburn relativo as álgebras associativas e o usamos na teoria de representações (bimódulos) de álgebras associativas para obter uma bela equivalência de categorias. Na seção 2.2, apresentamos e demostramos o Teorema de coordenatização de Zorn relativo as álgebras alternativas não-associativas. Esse teorema foi deixado como um exercício no livro [19], e o usamos na teoria de representações de álgebras alternativas para obter novamente uma bela equivalência de categorias, do qual, obtemos a classificação os bimódulos alternativos irredutíveis unitários sobre a álgebra matricial de Cayley-Dickson $C(F)$. Na seção 2.3, obtemos que o Teorema de coordenatização de Wedderburn, no caso $n \geq 3$, admite uma generalização formal para as álgebras alternativas de dimensão e característica arbitrária, o qual, usamos para demostrar (via extensão cisão nula) que cada bimódulo alternativo unitário para $M_{n}(F)$ com $n \geq 3$, é associativo. Esse resultado é análogo ao teorema 11.1 de [3], demostrado por N. Jacobson para corpos de característica diferente de 2.

O capítulo 3 é dedicado ao problema de Nathan Jacobson e suas soluções. Na seção 3.1, incluímos definições, exemplos e definimos um classe importante de álgebras alternativas (a álgebra dos quaterniões $\mathbb{H}$ ) que é usada ao longo do capítulo. Na seção 3.2, formulamos o problema de N. Jacobson. Esse problema, refere-se à descrição das álgebras alternativas que contém uma subálgebra $M_{2}(F)$ (álgebra split dos quaterniões) com o mesmo elemento identidade, o qual, foi formulado por N. Jacobson na década de 50 em [2, 3]. Na seção 3.4, descrevemos as soluções desse problema, os quais são obtidas ao longo da seção 3.3.

O capítulo 4 é dedicado as superálgebras alternativas simples e demostramos um Teorema de Fatorização de Kronecker para essas superálgebras. Na seção 4.1, incluímos definições, exemplos e alguns resultados principais sem apresentar demonstração da classificação das superálgebras associativas simples de dimensão finita sobre corpos algebricamente fechados, obtida por C. T. Wall [18], e a classificação das superálgebras alternativas simples de característica 2, obtida por I. Shestakov [16]. Na seção 4.2, formulamos um análogo do problema de Jacobson (dada no capítulo 3) para as superálgebras alternativas. Na seção 4.3, damos uma demonstração independente que funciona para o caso geral do clássico Teorema de Fatorização de Jacobson. Generalizamos esse resultado para obter um Teorema de Fatorização de Kronecker para as superálgebras alternativas cuja parte par contem a álgebra de Cayley-Dickson com o mesmo elemento identidade. Também, demostramos um Teorema de Fatorização de Kronecker para as superálgebras alternativas que contém $M_{(1 \mid 1)}(F)$ com o mesmo elemento identidade, o qual usamos para responder o análogo do problema de Jacobson para as superálgebras alternativas que foi formulado na seção 4.2. Finalmente, 
demonstramos um Teorema de Fatorização de Kronecker para as superálgebras alternativas simples $\mathbb{O}(4 \mid 4)$ e $\mathbb{O}[u]$.

Convém esclarecer ao leitor que, entre os resultados que aparecem na tese, todos aqueles que têm demonstração (a menos do Teorema 3) são contribuições nossas. 


\section{Capítulo 1}

\section{Algumas (super)álgebras não associativas}

Ao longo de cada capítulo vamos incluir definições necessárias para oferecer um conteúdo próprio de cada um deles. Assim, neste capítulo, apresentamos definições de algumas classes de (super)álgebras que serão estudadas nos próximos capítulos. As referências e os conteúdos podem ser encontrados em [6, 12, 15, 19].

\section{1 Álgebras e álgebras livres}

Definição 1 Seja $\mathcal{A}$ um espaço vetorial sobre um corpo $F$. O espaço vetorial $\mathcal{A}$ é uma álgebra sobre o corpo $F$ (ou $F$ - álgebra) se existe uma aplicação bilinear sobre $\mathcal{A}$, isto é, a aplicação $(x, y) \longmapsto x y$ de $\mathcal{A} \times \mathcal{A}$ em $\mathcal{A}$ dada com as seguintes condições

$$
(\alpha x+\beta y) z=\alpha(x z)+\beta(y z) ; x(\alpha y+\beta z)=\alpha(x y)+\beta(x z),
$$

para todo $\alpha, \beta \in F ; x, y, z \in \mathcal{A}$.

A aplicação bilinear sobre $\mathcal{A}$ na definição acima é chamada de multiplicação.

A álgebra $\mathcal{A}$ é chamada associativa se, a multiplicação é associativa, isto é, $(x y) z=$ $x(y z)$ para todo $x, y, z \in \mathcal{A}$. Se a multiplicação de $\mathcal{A}$ não satisfaz a condição anterior, a álgebra $\mathcal{A}$ é chamada de não associativa.

Ao considerar álgebras não associativas, a noção de um associador será útil $(x, y, z) \equiv$ $(x y) z-x(y z)$. Além disso, consideramos o comutador $[x, y] \equiv x y-y x$ dos elementos $x, y \in \mathcal{A}$.

Uma $F$-álgebra $\mathcal{L}$ é chamada uma álgebra de Lie, se sua multiplicação é anticomutativa, isto é,

$$
x^{2}=0
$$

e se ela satisfaz a identidade de Jacobi

$$
J(x, y, z) \equiv(x y) z+(y z) x+(z x) y=0 .
$$

As álgebras que satisfazem as seguintes identidades

$$
x y=y x, \quad x^{2}(y x)=\left(x^{2} y\right) x
$$

são chamadas álgebras de Jordan. As álgebras alternativas estão definidas pelas iden- 
tidades

$$
x^{2} y=x(x y), \quad y x^{2}=(y x) x .
$$

Sejam $\mathcal{A}$ e $\mathcal{B}$ álgebras sobre $F$. O produto de Kronecker $\mathcal{A} \otimes_{F} \mathcal{B}$ (escrito $\mathcal{A} \otimes \mathcal{B}$ se não houver ambiguidade) é o produto tensorial $\mathcal{A} \otimes_{F} \mathcal{B}$ dos espaços vetoriais $\mathcal{A}, \mathcal{B}$ (assim, todos os elementos são somas $\sum a \otimes b \operatorname{com} a \operatorname{em~} \mathcal{A}$ e $b$ em $\mathcal{B}$ ), sendo a multiplicação definida pela distributividade e

$$
\left(a_{1} \otimes b_{1}\right)\left(a_{2} \otimes b_{2}\right)=\left(a_{1} a_{2}\right) \otimes\left(b_{1} b_{2}\right)
$$

para $a_{i}$ em $\mathcal{A}$ e $b_{i}$ em $\mathcal{B}$. Se $\mathcal{A}$ tem identidade 1 , então o conjunto de todos os elementos $1 \otimes b \mathrm{em} \mathcal{A} \otimes \mathcal{B}$ é uma subálgebra de $\mathcal{A} \otimes \mathcal{B}$ que é isomorfa a $\mathcal{B}$, e podemos identificá-la com $\mathcal{B}$ (similarmente, se $\mathcal{B}$ tem identidade 1 , então $\mathcal{A} \otimes \mathcal{B}$ contém $\mathcal{A}$ como uma subálgebra).

Se $\mathcal{A}$ é um corpo $K$ (uma extensão arbitrária de $F$ ). Então, $K$ contém 1 , assim $\mathcal{B}_{K}=$ $K \otimes_{F} \mathcal{B}$ contém $\mathcal{B}$ (no sentido de isomorfismo) como uma subálgebra sobre $F$. Além disso, $\mathcal{B}_{K}$ é facilmente visto como uma álgebra sobre $K$, a qual, é chamada a extensão escalar de $\mathcal{B}$ para uma álgebra sobre $K$.

Seja $\mathcal{A}$ uma álgebra arbitrária. Os seguintes subconjuntos serão considerados na álgebra $\mathcal{A}$ : O centro associativo $N(\mathcal{A})$, o centro comutativo $K(\mathcal{A})$ e o centro $Z(A)$. Esses subconjuntos, respectivamente estão definidos da seguinte maneira:

$$
\begin{gathered}
N(\mathcal{A})=\{a \in \mathcal{A} \mid(a, \mathcal{A}, \mathcal{A})=(\mathcal{A}, a, \mathcal{A})=(\mathcal{A}, \mathcal{A}, a)=0\}, \\
K(\mathcal{A})=\{a \in \mathcal{A} \mid[a, \mathcal{A}]=0\}, \\
Z(\mathcal{A})=N(\mathcal{A}) \cap K(\mathcal{A}) .
\end{gathered}
$$

Seja a qualquer elemento de uma álgebra $\mathcal{A}$. A multiplicação à direita $R_{a}$ de $\mathcal{A}$ determinada por $a$, está definida por $R_{a}: x \longrightarrow x a$, para todo $x$ em $\mathcal{A}$. Claramente $R_{a}$ é um operador linear sobre $\mathcal{A}$. $\mathrm{O}$ conjunto de todas as multiplicações à direita de $\mathcal{A}$ é um subespaço da álgebra associativa $\operatorname{End}_{F}(\mathcal{A})$ de todos os operadores lineares sobre $\mathcal{A}$ pois, $a \longrightarrow R_{a}$ é uma aplicação linear de $\mathcal{A}$ em $\operatorname{End}_{F}(\mathcal{A})$. Por $R(\mathcal{A})$, denotamos a subálgebra de $\operatorname{End}_{F}(\mathcal{A})$ gerada por todos os $R_{a}$, e é chamada a álgebra de multiplicações à direita. De maneira semelhante, a multiplicação à esquerda $L_{a}$ definida por $L_{a}: x \longrightarrow$ ax para todo $x$ em $\mathcal{A}$, é um operador linear sobre $\mathcal{A}$, a aplicação $a \longrightarrow L_{a}$ é linear e o conjunto de todas as multiplicações à esquerda de $\mathcal{A}$, é um subespaço de $\operatorname{End}_{F}(\mathcal{A})$. Por $L(\mathcal{A})$, denotamos a subálgebra de $\operatorname{End}_{F}(\mathcal{A})$ gerada por todos os $L_{a}$, e é chamada a álgebra de multiplicações à esquerda.

Seja $X$ um conjunto arbitrário de símbolos. Em [19] foi construída a $F$-álgebra $F[X]$, a qual, chama-se de álgebra livre sobre $F$ do conjunto de geradores $X$. As álgebras livres possuem a propriedade universal dada no seguinte

Teorema 1 Seja $\mathcal{A}$ uma $F$-álgebra arbitrária e $\theta$ uma aplicação de $X$ em $\mathcal{A}$. Então, $\theta$ se estende de modo único a um homomorfismo da álgebra $F[X]$ na álgebra $\mathcal{A}$.

Os elementos da álgebra $F[X]$ são chamados de polinômios não-associativos dos elementos do conjunto $X$.

\section{2 Álgebras livres em uma variedade}

Sejam $X=\left\{x_{1}, x_{2}, \ldots\right\}$ um conjunto enumerável de símbolos e $f$ um elemento arbitrário de $F[X]$. Nesta, aparece apenas um quantidade finita de elementos de $X$, por exemplo $x_{1}, x_{2}, \ldots, x_{n}$ e escrevemos $f=f\left(x_{1}, x_{2}, \ldots, x_{n}\right)$. 
Dados $a_{1}, a_{2}, \ldots, a_{n}$ elementos de uma $F$-álgebra $\mathcal{A}$, pelo Teorema 1 , existe um único homomorfismo $\theta: F[X] \longrightarrow \mathcal{A}$, tal que, $\theta\left(x_{i}\right)=a_{i}$ para todo $i=1,2 \ldots, n$ e $\theta\left(x_{i}\right)=0$ para $i \neq 1,2, \ldots, n$. Denotemos por $f\left(a_{1}, a_{2}, \ldots, a_{n}\right)$ a imagem do elemento $f$ pelo homomorfismo $\theta$ e dizemos que o elemento $f\left(a_{1}, a_{2}, \ldots, a_{n}\right)$ é obtido por substituição dos elementos $a_{1}, a_{2}, \ldots, a_{n}$ no polinômio não-associativo $f\left(x_{1}, x_{2}, \ldots, x_{n}\right)$.

Um polinômio não-associativo $f=f\left(x_{1}, x_{2}, \ldots, x_{n}\right) \in F[X]$ é chamado de identidade de uma álgebra $\mathcal{A}$ se $f\left(a_{1}, a_{2}, \ldots, a_{n}\right)=0$ para quaisquer $a_{1}, a_{2}, \ldots, a_{n} \in \mathcal{A}$. Neste caso, também se diz que $\mathcal{A}$ satisfaz a identidade $f$ ou que $f$ é válida em $\mathcal{A}$.

A coleção de todas as identidades de uma álgebra $\mathcal{A}$ é um ideal da álgebra $F[X]$, que é chamado de ideal das identidades ou T-ideal da álgebra $\mathcal{A}$ e é denotado por $T(\mathcal{A})$. A coleção de todas as identidades que são satisfeitas por cada álgebra de uma classe de álgebras $\mathfrak{M}$ também é um ideal de $F[X]$, chamado ideal das identidades ou T-ideal da classe das álgebras $\mathfrak{M}$ e é denotado por $T(\mathfrak{M})$.

Dado um subconjunto $I$ de $F[X]$, a classe $\mathfrak{M}$ de todas as $F$-álgebras satisfazendo cada identidade de $I$ é chamada de variedade de $F$-álgebras definida pelo conjunto de identidades $I$, e assim, $I$ é dito de $\boldsymbol{i d e n t i d a d e s}$ definindo a variedade $\mathfrak{M}$.

Pela seção 1.1, a classe de todas as $F$-álgebras associativas é uma variedade com identidade $f=\left(x_{1} x_{2}\right) x_{3}-x_{1}\left(x_{2} x_{3}\right)$. A classe de todas as $F$-álgebras de Lie é uma variedade com as identidades $f=x_{1}^{2}$ e $g=J\left(x_{1}, x_{2}, x_{3}\right)$. A classe de todas as álgebras de Jordan é uma variedade com as identidades $f=\left[x_{1}, x_{2}\right]$ e $g=x_{1}^{2}\left(x_{2} x_{1}\right)-\left(x_{1}^{2} x_{2}\right) x_{1}$ e, todas as $F$-álgebras alternativas formam uma variedade com as identidades $f=x_{1}^{2} x_{2}-x_{1}\left(x_{1} x_{2}\right)$ e $g=x_{2} x_{1}^{2}-\left(x_{2} x_{1}\right) x_{1}$. Vamos denotar a variedade das álgebras associativas, a variedade das aĺgebras de Lie, a variedade das álgebras de Jordan e alternativas, respectivamente, por Ass, Lie, Jord e Alt.

Uma zero-álgebra é uma álgebra em que o produto de quaisquer dos elementos é zero; a variedade consistindo somente de zero-álgebras recebe o nome de variedade trivial.

Seja $\mathfrak{M}$ uma variedade não trivial de $F$-álgebras e $\mathcal{A}$ uma álgebra de $\mathfrak{M}$ com conjunto de geradores $Y$. A álgebra $\mathcal{A}$ é chamada de livre $\boldsymbol{n a}$ variedade $\mathfrak{M}$ (ou $\mathfrak{M}$-livre) com conjunto de geradores livres $Y$ se $Y$ gera $\mathcal{A}$ e se toda aplicação do conjunto $Y$ em uma álgebra arbitrária $\mathcal{B}$ de $\mathfrak{M}$ pode ser estendida de modo único a um homomorfismo da álgebra $\mathcal{A}$ em $\mathcal{B}$.

Dado um conjunto de polinômios $I$ de $F[X]$, o ideal da $F$-álgebra $\mathcal{A}$ gerado por todos os elementos da forma $f\left(a_{1}, a_{2}, \ldots, a_{n}\right)$, onde $f \in I$ e $a_{1}, a_{2}, \ldots, a_{n} \in \mathcal{A}$, será denotada por $I(\mathcal{A})$.

Teorema 2 Seja $\mathfrak{M}$ uma variedade não trivial de F-álgebras com sistema de identidades I. Então, para qualquer conjunto $Y$, a restrição do homomorfismo canônico $\sigma$ a $Y$ é injetiva, onde

$$
\sigma: F[Y] \longrightarrow F[Y] / I(F[Y]) .
$$

Além disso, $F_{\mathfrak{M}}[\sigma(Y)]=F[Y] / I(F[Y])$ é livre na variedade $\mathfrak{M}$ com conjunto de geradores livres $\sigma(Y)$. E também, quaisquer duas álgebras livres de $\mathfrak{M}$ com conjuntos equivalentes de geradores livres são isomorfas.

\subsection{Bimódulos}

As noções de um bimódulo tem papel importante na teoria das álgebras. Seja M uma variedade de álgebras sobre o corpo $F$. Suponha que, para uma álgebra $\mathcal{A}$ em $\mathfrak{M}$ e um espaço vetorial $V$ sobre $F$, estão definidas as composições bilineares $\mathcal{A} \times V \longrightarrow V$ e $V \times \mathcal{A} \longrightarrow V$, 
escritas como av e $v a$, para $a \in \mathcal{A}$ e $v \in V$. Então, o espaço vetorial $\mathcal{A} \oplus V$ é uma álgebra com a seguinte multiplicação

$$
(a+v)(b+w)=a b+(v b+a w)
$$

onde $a, b \in \mathcal{A}, v, w \in V$. A álgebra $\mathcal{A} \oplus V$ é chamada a extensão cisão nula da álgebra $\mathcal{A}$ pelo espaço $V$. Se a álgebra $\mathcal{A} \oplus V$ pertence à variedade $\mathfrak{M}$, então $V$ é chamado um bimódulo sobre a álgebra $\mathcal{A}$ (ou um $\mathcal{A}$-bimódulo) na variedade $\mathfrak{M}$.

Exemplo 1 Se $\mathfrak{M}$ é a variedade das álgebras associativas Ass, então as operações devem satisfazer as seguintes condições:

$$
(v a) b=v(a b), \quad(a v) b=a(v b), \quad(a b) v=a(b v),
$$

para todo $a, b \in \mathcal{A}, v \in V$. Na variedade das álgebras de Lie Lie, as correspondentes condições para as operações do bimódulo são da forma

$$
a v=-v a, \quad v(a b)=(v a) b-(v b) a .
$$

A variedade das álgebras alternativas Alt está definida via as seguintes identidades:

$$
(x, x, y)=0, \quad(x, y, y)=0 .
$$

Portanto, um bimódulo $V$ sobre uma álgebra alternativa $\mathcal{A}$ é alternativa se, e somente se, as seguintes relações são válidas na extensão cisão nula $\mathcal{A} \oplus V$ :

$$
(a+v, a+v, b+w)=0, \quad(a+v, b+w, b+w)=0,
$$

para todo $a, b \in \mathcal{A}, v, w \in V$. Como $V^{2}=0$ (na álgebra $\mathcal{A} \oplus V$ ), essas relações nos fornecem as seguintes condições para que um bimódulo $V$ seja alternativa:

$$
\begin{aligned}
& (a, a, v)=0, \quad(a, v, b)+(v, a, b)=0 \\
& (v, b, b)=0, \quad(a, v, b)+(a, b, v)=0 .
\end{aligned}
$$

Finalmente, a variedade Jord está definida pelas seguintes identidades:

$$
x y=y x, \quad\left(x^{2}, y, x\right)=0 .
$$

Se $\mathcal{J}$ é uma álgebra de Jordan e $V$ é um bimódulo sobre $\mathcal{J}$, então a álgebra $\mathcal{J} \oplus V$ é de Jordan se, e somente se, as seguintes relações são válidas:

$$
(a+v)(b+w)=(b+w)(a+v) ; \quad\left((a+v)^{2}, b+w, a+v\right)=0,
$$

para todo $a, b \in \mathcal{J} ; v, w \in V$. É fácil ver que essas relações são equivalentes aos seguintes:

$$
a v=v a, \quad\left(a^{2}, v, a\right)=0, \quad\left(a^{2}, b, v\right)+2(a v, b, a)=0,
$$

para todo $a, b \in \mathcal{J}, v \in V$.

Um endomorfismo do espaço vetorial $\mathcal{A}$ é chamada uma involução da álgebra $\mathcal{A}$ se, é de período 2 e, é um anti-homomorfismo.

Vamos a introduzir a noção de um bimódulo com involução. 
Seja $\mathcal{A}$ uma álgebra na variedade $\mathfrak{M}$ com involução "_". Então, um bimódulo $V$ para $\mathcal{A}$ na variedade $\mathfrak{M}$, é um bimódulo com involução, se existe um endomorfismo $v \longmapsto v^{*}$ de período 2 de $V$, tal que,

$$
(a v)^{*}=v^{*} \bar{a}, \quad(v a)^{*}=\bar{a} v^{*}
$$

para todo $a \in \mathcal{A}, v \in V$. O endomorfismo $v \longmapsto v^{*}$ é chamado a involução de $V$.

Se $V$ é um bimódulo com involução sobre $\mathcal{A}$, então junto com a involução de $\mathcal{A}$, podemos obter uma involução na extensão cisão nula $E=\mathcal{A} \oplus V$. Inversamente, qualquer involução em $E$ que estende a involução de $\mathcal{A}$, pode ser usada para fazer do bimódulo $V$, um bimódulo com involução.

\subsection{Superálgebras}

Definição 2 Uma álgebra $\mathcal{A}$ é chamada uma álgebra $\mathbb{Z}_{2}$-graduada ou uma superálgebra, se $\mathcal{A}=\mathcal{A}_{\overline{0}} \oplus \mathcal{A}_{\overline{1}}$, onde $\mathcal{A}_{i} \mathcal{A}_{j} \subseteq \mathcal{A}_{i+j}, i, j \in \mathbb{Z}_{2}$.

Exemplo 2 Seja $\Gamma$ a álgebra de Grassmann sobre um corpo $F$ gerada pelos elementos $1, e_{1}, \ldots, e_{n}, \ldots$ tais que

$$
e_{i}^{2}=0, \quad e_{i} e_{j}=-e_{j} e_{i}
$$

Os produtos

$$
1, \quad e_{i_{1}} e_{i_{2}} \ldots e_{i_{k}}, \quad i_{1}<i_{2}<\cdots<i_{k},
$$

formam uma base de $\Gamma$ sobre $F$ (consideremos 1 como o produto do conjunto vazio dos elementos $e_{i}$ ). Denote-se por $\Gamma_{\overline{0}}$ e $\Gamma_{\overline{1}}$ os espaços gerados pelos produtos de comprimento par e impar; então $\Gamma$ é a soma direta desses espaços,

$$
\Gamma=\Gamma_{\overline{0}} \oplus \Gamma_{\overline{1}},
$$

onde

$$
\Gamma_{i} \Gamma_{j} \subseteq \Gamma_{i+j(\bmod 2)}, \quad i, i \in\{\overline{0}, \overline{1}\}
$$

Em outras palavras, $\Gamma$ é uma álgebra $\mathbb{Z}_{2}$-graduada (ou superálgebra) sobre $F$.

Seja $\mathfrak{M}$ uma variedade de álgebras sobre um corpo infinito $F$, definida por algum sistema de identidades.

Definição 3 A superálgebra $\mathcal{A}=\mathcal{A}_{\overline{0}} \oplus \mathcal{A}_{\overline{1}}$ é chamada uma $\mathfrak{M}$-superálgebra se sua envolvente de Grassmann $\Gamma(\mathcal{A})=\Gamma_{\overline{0}} \otimes \mathcal{A}_{\overline{0}} \oplus \Gamma_{\overline{1}} \otimes \mathcal{A}_{\overline{1}}$ pertence a $\mathfrak{M}$.

Exemplo 3 A superálgebra de Grassmann $\Gamma$ é uma superálgebra comutativa, pois sua envolvente de Grassmann $\Gamma(\Gamma)=\Gamma_{\overline{0}} \otimes \Gamma_{\overline{0}}+\Gamma_{\overline{1}} \otimes \Gamma_{\overline{1}}$ é comutativa. Isto vem do fato de que $\Gamma$ satisfaz a seguinte identidade de comutatividade (ou supercomutatividade) graduada

$$
a b=(-1)^{|a||b|} b a
$$

onde $a, b \in \Gamma_{\overline{0}} \cup \Gamma_{\overline{1}} e|x|$ denota a paridade de um elemento homogêneo $x$ of $A:|x|=i$ se $x \in A_{i}$.

Se as identidades que definem à variedade $\mathfrak{M}$ são conhecidas, então é possível escrever as "superidentidades" que definem as $\mathfrak{M}$-superálgebras. 
Exemplo 4 Sejam $\mathcal{A}=\mathcal{A}_{\overline{0}} \oplus \mathcal{A}_{\overline{1}}$ uma superálgebra e $d \in \mathcal{A}_{\overline{0}}$, a, b, c $\in \mathcal{A}_{\overline{0}} \cup \mathcal{A}_{\overline{1}}$. A superálgebra $\mathcal{A}$ é uma superálgebra alternativa se satisfaz as seguintes identidades:

$$
\begin{gathered}
(a, b, c)+(-1)^{|b||c|}(a, c, b)=0 \\
(a, b, c)+(-1)^{|a||b|}(b, a, c)=0 \\
(d, d, a)=0 .
\end{gathered}
$$

Se a característica do corpo $F$ é diferente de 2 , note que a última identidade se segue das duas primeiras. Assim, também, as superálgebras de Lie estão definidas pelas seguintes identidades:

$$
\begin{gathered}
a b+(-1)^{|a||b|} b a=0, \\
(a b) c-a(b c)-(-1)^{|b||c|}(a c) b=0 ;
\end{gathered}
$$

e as superálgebras de Jordan estão definidas pelas seguintes identidades:

$$
\begin{gathered}
a b-(-1)^{|a||b|} b a=0, \\
(-1)^{|d|(|a|+|c|)}(a b, c, d)+(-1)^{|a|(|b|+|c|)}(b d, c, a)+(-1)^{|b|(|c|+|d|)}(d a, c, b)=0,
\end{gathered}
$$

onde $a, b, c, d \in \mathcal{A}_{0} \cup \mathcal{A}_{1}$.

Sejam $\mathcal{A}$ e $\mathcal{B}$ superálgebras sobre $F$. De modo análogo ao caso das álgebras, podemos definir o produto de Kronecker $\mathcal{A} \widetilde{\otimes}_{F} \mathcal{B}$ (escrito $\mathcal{A} \widetilde{\otimes} \mathcal{B}$ se não houver ambiguidade) como o produto tensorial graduado $\mathcal{A} \widetilde{\otimes}_{F} \mathcal{B}$ dos superespaços vetoriais $\mathcal{A}, \mathcal{B}$ (assim, todos os elementos são somas $\sum a \widetilde{\otimes} b \operatorname{com} a \mathrm{em} \mathcal{A}$ e $b$ em $\mathcal{B}$ ), sendo a multiplicação definida pela distributividade e

$$
\left(a_{1} \widetilde{\otimes} b_{1}\right)\left(a_{2} \widetilde{\otimes} b_{2}\right)=(-1)^{\left|b_{1}\right|\left|a_{2}\right|}\left(a_{1} a_{2}\right) \widetilde{\otimes}\left(b_{1} b_{2}\right)
$$

para $a_{i}$ em $\mathcal{A}$ e $b_{i}$ em $\mathcal{B}$. De forma semelhante, se $\mathcal{A}$ tem identidade 1 , então o conjunto de todos os elementos $1 \widetilde{\otimes} b$ em $\mathcal{A} \widetilde{\otimes} \mathcal{B}$ é uma subsuperálgebra de $\mathcal{A} \widetilde{\otimes} \mathcal{B}$ que é isomorfo a $\mathcal{B}$, e que podemos identificá-lo com $\mathcal{B}$ (similarmente, se $\mathcal{B}$ tem identidade 1 , então $\mathcal{A} \widetilde{\otimes} \mathcal{B}$ contém $\mathcal{A}$ como uma subsuperálgebra).

Seja $\mathfrak{M}$ uma arbitrária variedade de álgebras. Uma $\mathfrak{M}$-superálgebra $\mathcal{A}$ de dimensão finita é chamada semissimples se $\mathcal{A}$ é soma direta de ideais simples $\mathbb{Z}_{2}$-graduados.

Uma $\mathfrak{M}$-superálgebra $\mathcal{A}$ de dimensão finita é chamada separável se $\mathcal{A}_{K}=K \otimes_{F} \mathcal{A}$ é semissimples. 


\section{Capítulo 2}

\section{Teoremas de Coordenatização de Wedderburn e de Zorn}

Seja $\mathcal{A}$ uma álgebra associativa de dimensão finita sobre $F$. Então existe um ideal $\Re$, chamado o radical de $\mathcal{A}$, que é o único nilideal maximal de $\mathcal{A}$ (isto é, o ideal maximal que consiste inteiramente de elementos nilpotentes). Além disso, $\Re$ é nilpotente no sentido que existe um inteiro $t$ com a propriedade que qualquer produto $a_{1} a_{2} \ldots a_{t}$ de $t$ elementos de $\Re$ é zero; portanto $\Re$ é também o único ideal nilpotente maximal de $\mathcal{A}$. Módulo este radical, a álgebra é semissimples, isto é, a álgebra quociente $\mathcal{A} / \Re$ tem radical igual a zero. Além disso, qualquer álgebra associativa semissimples se escreve de maneira única como uma soma direta $I_{1} \oplus \cdots \oplus I_{r}$ de ideais bilaterais simples (onde uma álgebra é simples, desde que não tenha ideais próprios e não seja uma álgebra de dimensão 1 no qual o produto seja zero). Um célebre teorema de Wedderburn afirma que qualquer álgebra associativa simples $I$ é o produto de Kronecker $D \otimes_{F} M_{n}(F)$ de uma álgebra de divisão $D$ sobre $F$ e a álgebra de matrizes $M_{n}(F)$ de dimensão $n^{2}$, onde $n$ é único e $D$ está determinada de maneira única a menos de isomorfismo.

Outro resultado clássico nas álgebras associativas é o Teorema de Coordenatização de Wedderburn (Teorema 3), o qual afirma que qualquer álgebra associativa unitária que contém um sistema de $n^{2}$ elementos de matrizes unitárias, é também uma álgebra de matrizes.

Apresentamos o Teorema de Coordenatização de Wedderburn como bem disse K. McCrimmon, é o avô de todos os teoremas de Coordenatização. Além disso, apresentamos o Teorema de Coordenatização de Zorn que foi deixada como um exercício no livro [19]. Estes são exemplos de uma família de teoremas de coordenatização, os quais afirmam que uma álgebra será de uma certa forma, se conter uma família de elementos de um certo tipo.

\subsection{O Teorema de coordenatização de Wedderburn}

Definimos os $e_{i j}$ como as matrizes com 1 na $(i, j)$-entrada e 0 nas outras. Vamos chamar ao conjunto desses elementos, um sistema de matrizes unitárias que satisfazem

$$
e_{i j} e_{r s}=\delta_{j r} e_{i s}, \quad \sum e_{i i}=1
$$

onde $\delta$ é a delta de Kronecker.

Lembremos que uma álgebra $\mathcal{A}$ é associativa se, todos os associadores dos elementos de $\mathcal{A}$ são 0 , isto é, $(x, y, z)=0$ para todo $x, y, z \in \mathcal{A}$. 
Exemplo $5 \mathrm{~A}$ álgebra $M_{n}(F)$ de todas as matrizes $n \times n$ com entradas no corpo $F$, é uma álgebra associativa. Em geral, se $\mathcal{A}$ é uma álgebra associativa qualquer, podemos considerar a álgebra associativa $M_{n}(\mathcal{A})$ de todas as matrizes com coordenadas na álgebra $\mathcal{A}$.

Teorema 3 Seja $\mathcal{A}$ uma álgebra associativa com elemento identidade 1 tal que $\mathcal{A}$ contém um sistema de $n^{2}$ elementos de matrizes unitárias. Então, $\mathcal{A} \cong M_{n}(\mathcal{B})$ para uma certa subálgebra $\mathcal{B}$ de $\mathcal{A}$.

Demonstração: Fixemos $i, j, k, r, s \in\{1,2, \ldots, n\}$. Consideremos o conjunto $\mathcal{B}=\{a \in \mathcal{A}$ : $\left[a, e_{i j}\right]=0$ para todo $\left.i, j\right\}$. Pela seguinte identidade, válida em toda álgebra associativa,

$$
[x y, z]=x[y, z]+[x, z] y
$$

segue facilmente que o conjunto $\mathcal{B}$ é uma subálgebra de $\mathcal{A}$.

Seja $a \in \mathcal{A}$ e defina $a_{i j}=\sum_{k=1}^{n} e_{k i} a e_{j k}$. Então,

$$
a_{i j} e_{r s}=\left(\sum_{k=1}^{n} e_{k i} a e_{j k}\right) e_{r s}=\sum_{k=1}^{n} e_{k i} a\left(e_{j k} e_{r s}\right)=e_{r i} a e_{j s}
$$

e

$$
e_{r s} a_{i j}=e_{r s}\left(\sum_{k=1}^{n} e_{k i} a e_{j k}\right)=\sum_{k=1}^{n}\left(e_{r s} e_{k i}\right) a e_{j k}=e_{r i} a e_{j s} .
$$

$\operatorname{Logo}\left[a_{i j}, e_{r s}\right]=0$, e assim, $a_{i j} \in \mathcal{B}$. Além disso,

$$
\sum_{i, j=1}^{n} a_{i j} e_{i j}=\sum_{i, j=1}^{n} e_{i i} a e_{j j}=\left(\sum_{i=1}^{n} e_{i i}\right) a\left(\sum_{j=1}^{n} e_{j j}\right)=a .
$$

Agora suponha-se que os $b_{i j}$ são elementos de $\mathcal{B}$, tais que, $\sum_{i, j=1}^{n} b_{i j} e_{i j}=0$. Logo

$$
0=\sum_{k=1}^{n} e_{k p}\left(\sum_{i, j=1}^{n} b_{i j} e_{i j}\right) e_{q k}=b_{p q}
$$

para $p, q=1, \ldots, n$. Isto mostra que os elementos de $\mathcal{A}$ podem ser escritos de uma única forma $\sum_{i, j=1}^{n} a_{i j} e_{i j}$ onde $a_{i j} \in \mathcal{B}$ para todo $i, j$. Isto implica que, a aplicação $f: \mathcal{A} \longrightarrow M_{n}(\mathcal{B})$, definida por

$$
f(a)=\left[\begin{array}{cccc}
a_{11} & a_{12} & \cdots & a_{1 n} \\
a_{21} & a_{22} & \cdots & a_{2 n} \\
\vdots & \vdots & & \vdots \\
a_{n 1} & a_{n 2} & \cdots & a_{n n}
\end{array}\right]
$$

com $a=\sum_{i, j=1}^{n} a_{i j} e_{i j}$ é injetiva. É claro que $f$ também é sobrejetiva, e portanto, é um isomorfismo de espaços de $\mathcal{A}$ sobre $M_{n}(\mathcal{B})$.

Finalmente, vamos mostrar que $f$ é um homomorfismo de álgebras. Sejam $a=\sum_{i, j=1}^{n} a_{i j} e_{i j}$ e $b=\sum_{r, s=1}^{n} b_{r s} e_{r s} \operatorname{com} a_{i j}, b_{r s} \in \mathcal{B}$, então

$$
a b=\left(\sum_{i, j=1}^{n} a_{i j} e_{i j}\right)\left(\sum_{r, s=1}^{n} b_{r s} e_{r s}\right)=\sum_{i, j, r, s=1}^{n} a_{i j} b_{r s} e_{i j} e_{r s}=\sum_{i, j, s=1}^{n} a_{i j} b_{j s} e_{i s} .
$$


Assim,

$$
f(a b)=\left[\begin{array}{cccc}
\sum_{j=1}^{n} a_{1 j} b_{j 1} & \sum_{j=1}^{n} a_{1 j} b_{j 2} & \cdots & \sum_{j=1}^{n} a_{1 j} b_{j n} \\
\sum_{j=1}^{n} a_{2 j} b_{j 1} & \sum_{j=1}^{n} a_{2 j} b_{j 2} & \cdots & \sum_{j=1}^{n} a_{2 j} b_{j n} \\
\vdots & \vdots & & \vdots \\
\sum_{j=1}^{n} a_{n j} b_{j 1} & \sum_{j=1}^{n} a_{n j} b_{j 2} & \cdots & \sum_{j=1}^{n} a_{n j} b_{j n}
\end{array}\right]
$$

e é claro que o produto $f(a) f(b)$ é igual à matriz $f(a b)$. Portanto, $\mathcal{A} \cong M_{n}(\mathcal{B})$.

Isto prova o Teorema.

Q.E.D.

Corolário 4 (Teorema de coordenatização de Wedderburn) Seja $\mathcal{A}$ uma álgebra associativa, tal que, $\mathcal{A}$ contém uma subálgebra $M_{n}(F)$ como o mesmo elemento identidade. Então, $\mathcal{A} \cong \mathcal{B} \otimes M_{n}(F)$ para uma certa subálgebra $\mathcal{B}$ de $\mathcal{A}$ que comuta com todas as matrizes unitárias $e_{i j}$ de $M_{n}(F)$.

Se consideramos uma álgebra associativa unitária $\mathcal{B}$, podemos obter uma importante aplicação do Teorema de Coordenatização de Wedderburn na teoria de representações das álgebras associativas unitárias.

Corolário 5 A categoria dos bimódulos associativos unitários sobre $M_{n}(\mathcal{B})$ é equivalente à categoria dos bimódulos associativos unitários sobre $\mathcal{B}$.

Demonstração: Denotemos por $\operatorname{Bimod}_{A s s} \mathcal{B}$ a categoria dos bimódulos associativos unitários sobre $\mathcal{B}$ e por $\operatorname{Bimod}_{A s s} M_{n}(\mathcal{B})$ a categoria dos bimódulos associativos unitários sobre $M_{n}(\mathcal{B})$. Então, desejamos mostrar que

$$
\operatorname{Bimod}_{A s s} \mathcal{B} \simeq \operatorname{Bimod}_{A s s} M_{n}(\mathcal{B})
$$

Seja $N \in \operatorname{Obj}\left(\operatorname{Bimod}_{A s s} \mathcal{B}\right)$, e considere a extensão cisão nula $E=\mathcal{B} \oplus N$ da álgebra associativa $\mathcal{B}$ pelo bimódulo $N$. Como $E$ é uma álgebra associativa, podemos formar a álgebra matricial $K=M_{n}(E)$ que contém $M_{n}(\mathcal{B})$ como uma subálgebra. Além disso, $K$ contém o ideal $M=M_{n}(N) \cap K=M_{n}(N)$, que é o conjunto de matrizes de $K$ cujas entradas estão no ideal $N$ de $E$. Assim, $M_{n}(N)$ é um bimódulo associativo unitário para $M_{n}(\mathcal{B})$ relativo à multiplicação definida em $M_{n}(E)$. Vamos chamar $M$ ao $M_{n}(\mathcal{B})$-bimódulo associado com o dado bimódulo $N$ de $\mathcal{B}$ e denote

$$
M=\mathcal{F}(N) .
$$

Como $E=\mathcal{B} \oplus N$ temos que $K=M_{n}(\mathcal{B}) \oplus M$. Também $N^{2}=0$ em $E$, implica que $M^{2}=0$ em $K, \operatorname{assim} K$ é a extensão cisão nula de $M_{n}(\mathcal{B})$ por seu bimódulo $M$.

Pode-se verificar facilmente que $\mathcal{F}$ é realmente um funtor da categoria $\operatorname{Bimod}_{A s s} \mathcal{B}$ na

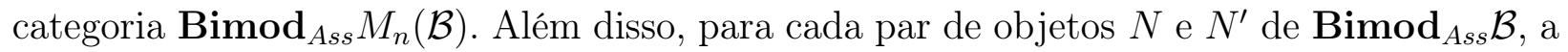
seguinte igualdade é válida:

$$
\mathcal{F}\left(\operatorname{Hom}\left(N, N^{\prime}\right)\right)=\operatorname{Hom}\left(\mathcal{F}(N), \mathcal{F}\left(N^{\prime}\right)\right)
$$

Portanto, $N$ e $N^{\prime}$ são isomorfos, se e somente se, $\mathcal{F}(N)$ e $\mathcal{F}\left(N^{\prime}\right)$ são isomorfos.

Similarmente, o funtor $\mathcal{F}$ oferece um isomorfismo reticulado do reticulado dos subbimódulos de $N$ relativo a $\mathcal{B}$ sobre o reticulado dos sub-bimódulos de $M$ sobre $M_{n}(\mathcal{B})$.

Para completar a redução da teoria dos bimódulos para $M_{n}(\mathcal{B})$ ao dos bimódulos para $\mathcal{B}$, agora mostraremos que cada $M_{n}(\mathcal{B})$-bimódulo unitário é isomorfo a algum bimódulo associado com um bimódulo unitário para $\mathcal{B}$. 
Considere-se um bimódulo associativo unitário $V$ para $M_{n}(\mathcal{B})$, e seja

$$
\mathcal{A}=M_{n}(\mathcal{B}) \oplus V
$$

a extensão cisão nula de $M_{n}(\mathcal{B})$ por $V$. Assim, $\mathcal{A}$ é uma álgebra associativa (com elemento identidade 1 , a identidade de $M_{n}(\mathcal{B})$ ) que contém a álgebra matricial $M_{n}(\mathcal{B})$ como uma subálgebra unitária, então pelo Teorema de Coordenatização de Wedderburn existe uma álgebra associativa $\mathcal{D}$ tal que $\mathcal{A}=M_{n}(\mathcal{D})$. Considere o conjunto $W$ dos elementos de $\mathcal{D}$ que aparecem nas entradas das matrizes de $V$. Logo

$$
V:=M_{n}(W)
$$

onde $W \triangleleft \mathcal{D}$ e $W^{2}=0$ em $\mathcal{D}$, pois $V \triangleleft \mathcal{A}$ e $V^{2}=0$ em $\mathcal{A}$, e assim, $\mathcal{D}=\mathcal{B} \oplus W$. Logo, $\mathcal{D}$ é a extensão cisão nula de $\mathcal{B}$ por seu bimódulo $W$, então $W$ é um bimódulo associativo unitário sobre $\mathcal{B}$. Portanto, $\mathcal{F}(W)=V$.

Isto prova o Corolário.

Q.E.D.

Do Corolário 5, podemos classificar os bimódulos associativos irredutíveis unitários sobre $M_{n}(F)$.

Corolário 6 Cada bimódulo associativo irredutivel unitário sobre $M_{n}(F)$, é isomorfo ao bimódulo regular $\operatorname{Reg}\left(M_{n}(F)\right)$.

\subsection{O Teorema de coordenatização de Zorn}

Na seção 2.1 temos descrito o Teorema de Coordenatização de Wedderburn e algumas de suas aplicações relacionadas às álgebras associativas unitárias. Nesta seção vamos apresentar e provar o Teorema de Coordenatização de Zorn, o qual, é o análogo do Teorema de Coordenatização de Wedderburn para as álgebras alternativas. Além disso, apresentaremos algumas das suas aplicações na teoria de representações das álgebras alternativas unitárias.

Definição 4 Uma álgebra alternativa (linear) é um espaço vetorial $\mathcal{A}$ com uma operação binária bilinear $(x, y) \mapsto x y$ satisfazendo as seguintes identidades:

$$
x^{2} y=x(x y), \quad(x y) y=x y^{2} .
$$

para todo $x, y \in \mathcal{A}$.

As equações anteriores são conhecidas, respectivamente, como identidade alternativa à esquerda e à direita. A linearização de (2.1) é

$$
(x \circ z) y=x(z y)+z(x y), \quad y(x \circ z)=(y x) z+(y z) x
$$

onde $x \circ x \equiv x z+z x$ é $\boldsymbol{o}$ produto de Jordan. As igualdades de (2.1) são equivalentes, em termos do associador, a

$$
(x, x, y)=(y, x, x)=0 \text { para todo } x \text { em } \mathcal{A}
$$

ou, em termos das multiplicações à direita e esquerda, a

$$
R_{x^{2}}=R_{x}^{2}, \quad L_{x^{2}}=L_{x}^{2} \text { para todo } x, y \text { em } \mathcal{A} \text {. }
$$


É claro que toda álgebra associativa é uma álgebra alternativa. Como tinhamos dito na introdução, o exemplo clássico de uma álgebra alternativa não-associativa é a álgebra de Octoniões $\mathbb{O}$ e a álgebra de Cayley-Dickson que vem do processo de duplicação de CayleyDickson (veja [19]).

A álgebra de matrizes de Cayley-Dickson $C(F)$ é uma álgebra alternativa nãoassociativa de dimensão 8 sobre $F$ com base $e_{i i}, e_{i j}^{(k)}(i, j=1,2 ; k=1,2,3)$ cuja tabela de multiplicação é a que segue:

$$
\begin{gathered}
e_{i i}^{2}=e_{i i}, \quad e_{i i} e_{j j}=0 ; \\
e_{i i} e_{i j}^{(k)}=e_{i j}^{(k)} e_{j j}=e_{i j}^{(k)}, \quad e_{j j} e_{i j}^{(k)}=e_{i j}^{(k)} e_{i i}=0 ; \\
\left(e_{i j}^{(k)}\right)^{2}=e_{i j}^{(k)} \circ e_{i j}^{(l)}=0 \quad(k \neq l) ; \\
e_{i j}^{(k)} e_{j i}^{(k)}=e_{i i}, \quad e_{i j}^{(k)} e_{j i}^{(l)}=0 \quad(k \neq l) ; \\
e_{i j}^{(k)} e_{i j}^{(k+1)}=(j-i) e_{j i}^{(k+2)}(k \bmod 3) .
\end{gathered}
$$

Os elementos $e_{i i}, e_{i j}^{(k)}$ são chamados matrizes unitárias de Cayley-Dickson.

A álgebra de matrizes de Cayley-Dickson, pode ser considerada não somente sobre corpos mas também sobre anéis associativos e comutativos $\Phi$. Segue imediatamente que a álgebra $C(\Phi)$ será também alternativa.

O nome "álgebra de matrizes" vem do fato de que os elementos de $C(F)$ podem ser representados por matrizes da seguinte maneira: Seja $a \in C(F)$ com

$$
a=\alpha_{11} e_{11}+\alpha_{22} e_{22}+\sum_{k=1}^{3}\left(\alpha_{12}^{(k)} e_{12}^{(k)}+\alpha_{21}^{(k)} e_{21}^{(k)}\right)
$$

Associamos ao elemento $a$ a matriz

$$
\left[\begin{array}{ll}
\alpha_{11} & a_{12} \\
a_{21} & \alpha_{22}
\end{array}\right] \text {, onde } a_{i j}=\left(\alpha_{i j}^{(1)}, \alpha_{i j}^{(2)}, \alpha_{i j}^{(3)}\right) \in F^{3} .
$$

A soma e a multiplicação escalar dos elementos da álgebra $C(F)$ correspondem às usuais soma e multiplicação escalar de matrizes da forma acima. No entanto, a multiplicação dos elementos da álgebra $C(F)$ correspondem a uma multiplicação especial (veja [19]).

Ao longo do nosso trabalho, vamos utilizar uma série de identidades que são válidas em cada álgebra alternativa, cada uma dessas identidades irão aparecer no momento em que elas forem necessárias.

Para começar, cada álgebra alternativa $\mathcal{A}$ satisfaz as seguintes identidades chamadas as identidades de Moufang

$$
\begin{gathered}
x(y z y)=[(x y) z] y, \text { identidade de Moufang à direita, } \\
(y z y) x=y[z(y x)], \text { identidade de Moufang à esquerda, } \\
(x y)(z x)=x(y z) x, \text { identidade de Moufang do meio. }
\end{gathered}
$$

Com isso, temos condições de anunciar e mostrar o Teorema de Coordenatização de Zorn relacionada às álgebras alternativas, o qual, foi formulado em [19] como um exercício: Seja $\mathcal{A}$ uma álgebra alternativa com elemento identidade 1 que contém um sistema de matrizes 
unitárias de Cayley-Dickson tais que $e_{11}+e_{22}=1$.

Lema 1 A álgebra $\mathcal{A}$ é decomponível numa soma direta de submódulos:

$$
\mathcal{A}=\mathcal{A}_{11} \oplus \mathcal{A}_{12} \oplus \mathcal{A}_{21} \oplus \mathcal{A}_{22}
$$

onde $\mathcal{A}_{i j}=\left\{a \in \mathcal{A}: e_{i i} a=a e_{j j}=a\right\}$. Além disso, as componentes $\mathcal{A}_{i j}$ possuem as relações

$$
\begin{gathered}
\mathcal{A}_{i i}^{2} \subseteq \mathcal{A}_{i i}, \quad \mathcal{A}_{i i} \mathcal{A}_{j j}=0 \\
\mathcal{A}_{i i} \mathcal{A}_{i j}+\mathcal{A}_{i j} \mathcal{A}_{j j} \subseteq \mathcal{A}_{i j}, \quad \mathcal{A}_{j j} \mathcal{A}_{i j}=\mathcal{A}_{i j} \mathcal{A}_{i i}=0 \\
\mathcal{A}_{i j} \mathcal{A}_{i j} \subseteq \mathcal{A}_{j i}, \quad \mathcal{A}_{i j} \mathcal{A}_{j i} \subseteq \mathcal{A}_{i i}
\end{gathered}
$$

onde $i \neq j$.

Demonstração: Seja $a \in \mathcal{A}$ então, pela identidade alternativa à direita, temos $\left(a, e_{i i}, e_{i i}\right)=$ 0 . Se $i \neq j$, então

$$
\begin{aligned}
\left(a, e_{i i}, e_{j j}\right) & =\left(a e_{i i}\right) e_{j j}=\left(a e_{i i}\right) e_{j j}^{2}=\left(a, e_{i i}, e_{j j}\right) e_{j j} \\
& =-\left(a, e_{j j}, e_{i i}\right) e_{j j}=-\left(\left(a e_{j j}\right) e_{i i}\right) e_{j j} \stackrel{(2.8)}{=}-a\left(e_{j j} e_{i i} e_{j j}\right)=0 .
\end{aligned}
$$

Assim,

$$
a=1 a 1=\left(e_{11}+e_{22}\right) a\left(e_{11}+e_{22}\right)=e_{11} a e_{11}+e_{11} a e_{22}+e_{22} a e_{11}+e_{22} a e_{22}
$$

e $e_{i i} a e_{j j} \in \mathcal{A}_{i j}(i, j=1,2)$ pois

$$
e_{i i}\left(e_{i i} a e_{j j}\right)=e_{i i}^{2}\left(a e_{j j}\right)=e_{i i} a e_{j j} \text { e }\left(e_{i i} a e_{j j}\right) e_{j j}=\left(e_{i i} a\right) e_{j j}^{2}=e_{i i} a e_{j j} .
$$

Logo, $a \in \mathcal{A}_{11}+\mathcal{A}_{12}+\mathcal{A}_{21}+\mathcal{A}_{22}$, e assim, $\mathcal{A} \subseteq \mathcal{A}_{11}+\mathcal{A}_{12}+\mathcal{A}_{21}+\mathcal{A}_{22}$. Portanto

$$
\mathcal{A}=\mathcal{A}_{11}+\mathcal{A}_{12}+\mathcal{A}_{21}+\mathcal{A}_{22}
$$

Suponhamos que existem elementos $a_{i j} \in \mathcal{A}_{i j}$, tais que, $\sum a_{i j}=0$. Logo

$$
0=\sum\left(e_{r r} a_{i j}\right) e_{s s}=a_{r s}
$$

para $r, s=1,2$. Consequentemente

$$
\mathcal{A}=\mathcal{A}_{11} \oplus \mathcal{A}_{12} \oplus \mathcal{A}_{21} \oplus \mathcal{A}_{22}
$$

Agora, vamos provar as relações. Sejam $a, b \in \mathcal{A}_{i i}$ então, por (2.2)

$$
\begin{gathered}
e_{i i}(a b)=\left(e_{i i} \circ a\right) b-a\left(e_{i i} b\right)=2 a b-a b=a b \quad \mathrm{e} \\
(a b) e_{i i}=a\left(b \circ e_{i i}\right)-\left(a e_{i i}\right) b=2 a b-a b=a b .
\end{gathered}
$$

Portanto, $a b \in \mathcal{A}_{i i}$, e assim $\mathcal{A}_{i i}^{2} \subseteq \mathcal{A}_{i i}$.

Consideremos a linearização da identidade de Moufang do meio (2.10)

$$
(x y)(z w)+(w y)(z x)-[w(y z)] x-[x(y z)] w=0 .
$$

Sejam $a \in \mathcal{A}_{i i}$ e $b \in \mathcal{A}_{j j}$. Então, usando os fatos $e_{i i} b=b e_{i i}=b\left(1-e_{j j}\right)=b-b e_{j j}=0$, 
$e_{j j} a=a e_{i i}=0$ e por $(2.11)$, temos

$$
a b=\left(a e_{i i}\right)\left(e_{j j} b\right)=-\left(b e_{i i}\right)\left(e_{j j} a\right)+\left[b\left(e_{i i} e_{j j}\right)\right] a+\left[a\left(e_{i i} e_{j j}\right)\right] b=0 .
$$

Isto implica que $\mathcal{A}_{i i} A_{j j}=0$.

Sejam $a \in \mathcal{A}_{i i}$ e $b \in \mathcal{A}_{i j}$ então, por $(2.2)$

$$
\begin{gathered}
e_{i i}(a b)=\left(e_{i i} \circ a\right) b-a\left(e_{i i} b\right)=2 a b-a b=a b \quad \mathrm{e} \\
(a b) e_{j j}=a\left(b \circ e_{j j}\right)-\left(a e_{j j}\right) b=a\left(b e_{j j}\right)+a\left(e_{j j} b\right)=a b .
\end{gathered}
$$

Consequentemente $a b \in \mathcal{A}_{i j}$ para quaisquer $a \in \mathcal{A}_{i i}$ e $b \in \mathcal{A}_{i j}$, e assim $\mathcal{A}_{i i} \mathcal{A}_{i j} \subseteq \mathcal{A}_{i j}$. Tomemos $a \in \mathcal{A}_{i j}$ e $b \in \mathcal{A}_{j j}$ então, novamente por (2.2)

$$
\begin{gathered}
e_{i i}(a b)=\left(e_{i i} \circ a\right) b-a\left(e_{i i} b\right)=\left(e_{i i} a\right) b+\left(a e_{i i}\right) b=a b \quad \mathrm{e} \\
(a b) e_{j j}=a\left(b \circ e_{j j}\right)-\left(a e_{j j}\right) b=2 a b-a b=a b .
\end{gathered}
$$

Logo, $a b \in \mathcal{A}_{i j}$ para quaisquer $a \in \mathcal{A}_{i j}$ e $b \in \mathcal{A}_{j j}$, e assim $\mathcal{A}_{i j} \mathcal{A}_{j j} \subseteq \mathcal{A}_{i j}$. Portanto, como $\mathcal{A}_{i i} \mathcal{A}_{i j} \subseteq \mathcal{A}_{i j}$ e $\mathcal{A}_{i j} \mathcal{A}_{j j} \subseteq \mathcal{A}_{i j}$, obtemos $\mathcal{A}_{i i} \mathcal{A}_{i j}+\mathcal{A}_{i j} \mathcal{A}_{j j} \subseteq \mathcal{A}_{i j}$

Sejam $a \in \mathcal{A}_{j j}, b \in \mathcal{A}_{i j}$ e $c \in \mathcal{A}_{i i}$ elementos arbitrários. Então, por (2.11) e o fato $e_{i i} a=c e_{j j}=0$, obtemos

$$
\begin{gathered}
a b=\left(a e_{j j}\right)\left(e_{i i} b\right)=-\left(b e_{j j}\right)\left(e_{i i} a\right)+\left[b\left(e_{j j} e_{i i}\right)\right] a+\left[a\left(e_{j j} e_{i i}\right)\right] b=0 \quad \mathrm{e} \\
b c=\left(b e_{j j}\right)\left(e_{i i} c\right)=-\left(c e_{j j}\right)\left(e_{i i} b\right)+\left[c\left(e_{j j} e_{i i}\right)\right] b+\left[b\left(e_{j j} e_{i i}\right)\right] c=0 .
\end{gathered}
$$

Portanto, $\mathcal{A}_{j j} \mathcal{A}_{i j}=\mathcal{A}_{i j} A_{i i}=0$.

Sejam $a, b \in \mathcal{A}_{i j}$ então, por (2.2)

$$
e_{j j}(a b)=\left(e_{j j} \circ a\right) b-a\left(e_{j j} b\right)=\left(e_{j j} a\right) b+\left(a e_{j j}\right) b=a b
$$

pois $e_{j j} b=0 \mathrm{e}$

$$
(a b) e_{i i}=a\left(b \circ e_{i i}\right)-\left(a e_{i i}\right) b=a b .
$$

Assim, $a b \in \mathcal{A}_{j i}$ para quaisquer $a, b \in \mathcal{A}_{i j}$, e portanto $\mathcal{A}_{i j} \mathcal{A}_{i j} \subseteq \mathcal{A}_{j i}$.

Finalmente, sejam $a \in \mathcal{A}_{i j}$ e $b \in \mathcal{A}_{j i}$ então, novamente por (2.2)

$$
\begin{gathered}
e_{i i}(a b)=\left(e_{i i} \circ a\right) b-a\left(e_{i i} b\right)=\left(e_{i i} a\right) b+\left(a e_{i i}\right) b=a b \quad \mathrm{e} \\
(a b) e_{i i}=a\left(b \circ e_{i i}\right)-\left(a e_{i i}\right) b=a\left(b e_{i i}\right)+a\left(e_{i i} b\right)=a b .
\end{gathered}
$$

Logo, $a b \in \mathcal{A}_{i i}$ para quaisquer $a \in \mathcal{A}_{i j}$ e $b \in \mathcal{A}_{j i}$, e portanto $\mathcal{A}_{i j} \mathcal{A}_{j i} \subseteq \mathcal{A}_{i i}$.

Isto prova o Lema.

Q.E.D.

\section{Lema 2 Para $i \neq j$}

$$
\mathcal{A}_{i j}=\mathcal{A}_{i j}^{(1)} \oplus \mathcal{A}_{i j}^{(2)} \oplus \mathcal{A}_{i j}^{(3)}
$$

onde $\mathcal{A}_{i j}^{(k)}=\mathcal{A}_{i i} e_{i j}^{(k)}=e_{i j}^{(k)} \mathcal{A}_{j j}, k=1,2,3$. Além disso, as componentes $\mathcal{A}_{i j}^{(k)}$ possuem as seguintes relações

$$
\begin{gathered}
\mathcal{A}_{i j}^{(k)} \mathcal{A}_{j i}^{(k)} \subseteq \mathcal{A}_{i i}, \quad \mathcal{A}_{i j}^{(k)} \mathcal{A}_{j i}^{(l)}=0, \quad k \neq l \\
\mathcal{A}_{i j}^{(k)} \mathcal{A}_{i j}^{(k)}=0, \quad \mathcal{A}_{i j}^{(k)} \mathcal{A}_{i j}^{(k+1)} \subseteq \mathcal{A}_{j i}^{(k+2)}, \quad k \bmod 3 .
\end{gathered}
$$


Demonstração: Pelas relações do Lema 1

$$
\begin{aligned}
\mathcal{A}_{i i} e_{i j}^{(k)} & =e_{i i}\left(\mathcal{A}_{i i} e_{i j}^{(k)}\right) \stackrel{(2.6)}{=}\left(e_{i j}^{(k)} e_{j i}^{(k)}\right)\left(\mathcal{A}_{i i} e_{i j}^{(k)}\right) \\
& \stackrel{(2.10)}{\subseteq} e_{i j}^{(k)}\left(\left(e_{j i}^{(k)} \mathcal{A}_{i i}\right) e_{i j}^{(k)}\right) \\
& \subseteq e_{i j}^{(k)} \mathcal{A}_{j j} .
\end{aligned}
$$

De maneira análoga, $e_{i j}^{(k)} \mathcal{A}_{j j} \subseteq \mathcal{A}_{i i} e_{i j}^{(k)}$. Logo, $\mathcal{A}_{i i} e_{i j}^{(k)}=e_{i j}^{(k)} \mathcal{A}_{j j}, k=1,2,3$. Portanto, $\mathcal{A}_{i j}^{(k)}$ está bem definida.

Em seguida, mostraremos que $\mathcal{A}_{i j}=\mathcal{A}_{i j}^{(1)}+\mathcal{A}_{i j}^{(2)}+\mathcal{A}_{i j}^{(3)}$. Sejam $a_{i i} \in \mathcal{A}_{i i}$ e $a_{j j} \in \mathcal{A}_{j j}$, então por $(2.2)$ e $(2.4)$

$$
\begin{aligned}
& e_{i i} a_{i j}^{(k)}=e_{i i}\left(a_{i i} e_{i j}^{(k)}\right)=\left(e_{i i} \circ a_{i i}\right) e_{i j}^{(k)}-a_{i i}\left(e_{i i} e_{i j}^{(k)}\right)=2 a_{i i} e_{i j}^{(k)}-a_{i i} e_{i j}^{(k)}=a_{i j}^{(k)} \mathrm{e} \\
& a_{i j}^{(k)} e_{j j}=\left(e_{i j}^{(k)} a_{j j}\right) e_{j j}=e_{i j}^{(k)}\left(a_{j j} \circ e_{j j}\right)-\left(e_{i j}^{(k)} e_{j j}\right) a_{j j}=2 e_{i j}^{(k)} a_{j j}-e_{i j}^{(k)} a_{j j}=a_{i j}^{(k)} .
\end{aligned}
$$

Assim $\mathcal{A}_{i j}^{(k)} \subseteq \mathcal{A}_{i j}$, o qual, implica imediatamente que $\mathcal{A}_{i j} \supseteq \mathcal{A}_{i j}^{(1)}+\mathcal{A}_{i j}^{(2)}+\mathcal{A}_{i j}^{(3)}$. Portanto, será suficiente mostrar a outra inclusão. É claro que por (2.4), os $e_{i j}^{(k)}$ são elementos de $\mathcal{A}_{i j}$. Seja $a_{i j} \in \mathcal{A}_{i j}$, então por (2.6) e (2.2), e pelas relações do Lema 1

$$
\begin{aligned}
a_{i j}=e_{i i} a_{i j}=\left(e_{i j}^{(1)} e_{j i}^{(1)}\right) a_{i j} & =e_{i j}^{(1)}\left(e_{j i}^{(1)} \circ a_{i j}\right)-\left(e_{i j}^{(1)} a_{i j}\right) e_{j i}^{(1)} \\
& =e_{i j}^{(1)}\left(e_{j i}^{(1)} a_{i j}\right)-\left(e_{i j}^{(1)} a_{i j}\right) e_{j i}^{(1)} \in e_{i j}^{(1)} A_{j j}+\mathcal{A}_{j i} e_{j i}^{(1)},
\end{aligned}
$$

onde $e_{i j}^{(1)} \mathcal{A}_{j j}=\mathcal{A}_{i j}^{(1)}$ e para todo $a_{j i} \in \mathcal{A}_{j i}$, temos por $(2.7)$

$$
\begin{aligned}
a_{j i} e_{j i}^{(1)}=(j-i) a_{j i}\left(e_{i j}^{(2)} e_{i j}^{(3)}\right) & =(j-i)\left(\left(a_{j i} \circ e_{i j}^{(2)}\right) e_{i j}^{(3)}-e_{i j}^{(2)}\left(a_{j i} e_{i j}^{(3)}\right)\right) \\
& =(j-i)\left(\left(e_{i j}^{(2)} a_{j i}\right) e_{i j}^{(3)}-e_{i j}^{(2)}\left(a_{j i} e_{i j}^{(3)}\right)\right) \in \mathcal{A}_{i i} e_{i j}^{(3)}+e_{i j}^{(2)} \mathcal{A}_{j j} .
\end{aligned}
$$

$\operatorname{Logo} a_{i j} \in \mathcal{A}_{i j}^{(2)}+\mathcal{A}_{i j}^{(3)}+\mathcal{A}_{i j}^{(1)}$, e assim $\mathcal{A}_{i j} \subseteq \mathcal{A}_{i j}^{(1)}+\mathcal{A}_{i j}^{(2)}+\mathcal{A}_{i j}^{(3)}$. Portanto,

$$
\mathcal{A}_{i j}=\mathcal{A}_{i j}^{(1)}+\mathcal{A}_{i j}^{(2)}+\mathcal{A}_{i j}^{(3)} .
$$

Agora, suponhamos que os $a_{i i}^{(k)}$ são elementos de $\mathcal{A}_{i i}$ tais que $\sum_{k} a_{i i}^{(k)} e_{i j}^{(k)}=0$. Então, por

$$
\begin{aligned}
0=\sum_{k}\left(a_{i i}^{(k)} e_{i j}^{(k)}\right) e_{j i}^{(l)} & =\sum_{k}\left[a_{i i}^{(k)}\left(e_{i j}^{(k)} \circ e_{j i}^{(l)}\right)-\left(a_{i i}^{(k)} e_{j i}^{(l)}\right) e_{i j}^{(k)}\right] \\
& =\sum_{k} a_{i i}^{(k)}\left(e_{i j}^{(k)} e_{j i}^{(l)}\right)=a_{i i}^{(l)}\left(e_{i j}^{(l)} e_{j i}^{(l)}\right)=a_{i i}^{(l)} e_{i i}=a_{i i}^{(l)},
\end{aligned}
$$

onde por (2.6) $e_{i j}^{(k)} e_{j i}^{(l)}=0$ se $k \neq l$. Isto mostra que os elementos de $\mathcal{A}_{i j}$ podem ser escritos de uma única forma, para todo $i, j$. Portanto, os coeficientes são únicos e a soma $\mathcal{A}_{i j}^{(1)}+\mathcal{A}_{i j}^{(2)}+\mathcal{A}_{i j}^{(3)}$ é direta.

Agora, vamos mostrar as relações. As inclusões $\mathcal{A}_{i j}^{(k)} \subseteq \mathcal{A}_{i j}, \mathcal{A}_{j i}^{(k)} \subseteq \mathcal{A}_{j i}$ e a relação $\mathcal{A}_{i j} \mathcal{A}_{j i} \subseteq \mathcal{A}_{i i}$ do Lema 1 , implicam que, $\mathcal{A}_{i j}^{(k)} \mathcal{A}_{j i}^{(k)} \subseteq \mathcal{A}_{i i}$.

Considere $a_{i i} e_{i j}^{(k)}=a_{i j}^{(k)} \in \mathcal{A}_{i j}^{(k)}$ e $b_{j j} e_{j i}^{(l)}=b_{j i}^{(l)} \in \mathcal{A}_{j i}^{(l)}(k \neq l)$. Então, por (2.11) e (2.6) e, 
o fato que $c_{i i} e_{i j}^{(k)}=e_{i j}^{(k)} b_{j j}$ para algum $c_{i i} \in \mathcal{A}_{i i}$

$$
\begin{aligned}
\left(a_{i i} e_{i j}^{(k)}\right)\left(b_{j j} e_{j i}^{(l)}\right) & =-\left(e_{j i}^{(l)} e_{i j}^{(k)}\right)\left(b_{j j} a_{i i}\right)+\left[e_{j i}^{(l)}\left(e_{i j}^{(k)} b_{j j}\right)\right] a_{i i}+\left[a_{i i}\left(e_{i j}^{(k)} b_{j j}\right)\right] e_{j i}^{(l)} \\
& =\left[-\left(a_{i i}, c_{i i}, e_{i j}^{(k)}\right)+\left(a_{i i} c_{i i}\right) e_{i j}^{(k)}\right] e_{j i}^{(l)} \\
& =\left[-\left(e_{i j}^{(k)}, a_{i i}, c_{i i}\right)+\left(a_{i i} c_{i i}\right) e_{i j}^{(k)}\right] e_{j i}^{(l)} \\
& =\left[\left(a_{i i} c_{i i}\right) e_{i j}^{(k)}\right] e_{j i}^{(l)} \\
& \stackrel{(2.2)}{=}\left(a_{i i} c_{i i}\right)\left(e_{i j}^{(k)} \circ e_{j i}^{(l)}\right)+\left(\left(a_{i i} c_{i i}\right) e_{j i}^{(l)}\right) e_{i j}^{(k)} \\
& =0 .
\end{aligned}
$$

$\operatorname{Assim}\left(a_{i i} e_{i j}^{(k)}\right)\left(b_{j j} e_{j i}^{(l)}\right)=0$, e portanto, $\mathcal{A}_{i j}^{(k)} \mathcal{A}_{j i}^{(l)}=0$ para $k \neq l$.

Para provar $\mathcal{A}_{i j}^{(k)} \mathcal{A}_{i j}^{(k)}=0$ considera-se $a_{i i} e_{i j}^{(k)}, b_{i i} e_{i j}^{(k)} \in \mathcal{A}_{i j}^{(k)}$. Então, por (2.11) e (2.5) tem-se

$$
\left(a_{i i} e_{i j}^{(k)}\right)\left(b_{i i} e_{i j}^{(k)}\right)=-\left(e_{i j}^{(k)} e_{i j}^{(k)}\right)\left(b_{i i} a_{i i}\right)+\left[e_{i j}^{(k)}\left(e_{i j}^{(k)} b_{i i}\right)\right] a_{i i}+\left[a_{i i}\left(e_{i j}^{(k)} b_{i i}\right)\right] e_{i j}^{(k)}=0 .
$$

Isto implica o que queríamos mostrar.

Finalmente, sejam $a_{i i} e_{i j}^{(k)} \in \mathcal{A}_{i j}^{(k)}$ e $b_{i i} e_{i j}^{(k+1)} \in \mathcal{A}_{i j}^{(k+1)}$. Então, novamente por (2.11)

$$
\begin{aligned}
\left(a_{i i} e_{i j}^{(k)}\right)\left(b_{i i} e_{i j}^{(k+1)}\right) & =-\left(e_{i j}^{(k+1)} e_{i j}^{(k)}\right)\left(b_{i i} a_{i i}\right)+\left[e_{i j}^{(k+1)}\left(e_{i j}^{(k)} b_{i i}\right)\right] a_{i i}+\left[a_{i i}\left(e_{i j}^{(k)} b_{i i}\right)\right] e_{i j}^{(k+1)} \\
= & \left(e_{i j}^{(k)} e_{i j}^{(k+1)}\right)\left(b_{i i} a_{i i}\right) \\
\stackrel{(2.7)}{=} & (j-i) e_{j i}^{(k+2)}\left(b_{i i} a_{i i}\right)(k \bmod 3)
\end{aligned}
$$

onde $b_{i i} a_{i i} \in \mathcal{A}_{i i}$ e $e_{j i}^{(k+2)}\left(b_{i i} a_{i i}\right) \in \mathcal{A}_{j i}^{(k+2)}$ para todo $a_{i i}, b_{i i} \in \mathcal{A}_{i i}$. Portanto,

$$
\mathcal{A}_{i j}^{(k)} \mathcal{A}_{i j}^{(k+1)} \subseteq \mathcal{A}_{j i}^{(k+2)}(k \bmod 3) .
$$

Isso prova o Lema.

Q.E.D.

Lema $3 O$ anel $\Omega=\mathcal{A}_{11}$ é um anel associativo e comutativo.

Demonstração: Fixemos elementos arbitrários $a_{11}, b_{11}, c_{11} \in \mathcal{A}_{11}$. Primeiramente provamos que o associador $\left(a_{11}, b_{11}, c_{11}\right)$ é anulado pelo elemento $e_{12}^{(1)}$. Então,

$$
\begin{aligned}
\left(a_{11}, b_{11}, c_{11}\right) e_{12}^{(1)} & =\left[\left(a_{11} b_{11}\right) c_{11}-a_{11}\left(b_{11} c_{11}\right)\right] e_{12}^{(1)} \\
& =\left[\left(a_{11} b_{11}\right) c_{11}\right] e_{12}^{(1)}-\left[a_{11}\left(b_{11} c_{11}\right)\right] e_{12}^{(1)} \\
& \stackrel{(2.2)}{=}\left[\left(a_{11} b_{11}\right) c_{11}\right] e_{12}^{(1)}-a_{11}\left(\left(b_{11} c_{11}\right) \circ e_{12}^{(1)}\right)+\left(a_{11} e_{12}^{(1)}\right)\left(\left(b_{11} c_{11}\right)\right. \\
& =\left[\left(a_{11} b_{11}\right) c_{11}\right] e_{12}^{(1)}-a_{11}\left(\left(b_{11} c_{11}\right) e_{12}^{(1)}\right)
\end{aligned}
$$

pois pelo Lema $1, \mathcal{A}_{12} \mathcal{A}_{11}=0$. Consideremos as linearizações da identidade de Moufang à direita

$$
\begin{gathered}
{[x(w z) y]+x[(y z) w]-[(x w) z] y-[(x y) z] w=0 \quad \text { ou }} \\
x[w(z y)]+x[y(z w)]-[(x w) z] y-[(x y) z] w=0 .
\end{gathered}
$$


A equação (2.12), pode-se escrever $[(x w) z] y-x[(w z) y]=x[(y z) w]-[(x y) z] w$. Então,

$$
\left[\left(a_{11} b_{11}\right) c_{11}\right] e_{12}^{(1)}-a_{11}\left[\left(b_{11} c_{11}\right) e_{12}^{(1)}\right]=a_{11}\left[\left(e_{12}^{(1)} c_{11}\right) b_{11}\right]-\left[\left(a_{11} e_{12}^{(1)}\right) c_{11}\right] b_{11}=0 .
$$

$\operatorname{Assim}\left(a_{11}, b_{11}, c_{11}\right) e_{12}^{(1)}=0$.

Agora, como $\left(a_{11}, b_{11}, c_{11}\right) \in A_{11}$, então por $(2.6)$

$$
\begin{aligned}
\left(a_{11}, b_{11}, c_{11}\right)=\left(a_{11}, b_{11}, c_{11}\right) e_{11} & =\left(a_{11}, b_{11}, c_{11}\right)\left(e_{12}^{(1)} e_{21}^{(1)}\right) \\
& \stackrel{(2.2)}{=}\left(\left(a_{11}, b_{11}, c_{11}\right) \circ e_{12}^{(1)}\right) e_{21}^{(1)}-e_{12}^{(1)}\left(\left(a_{11}, b_{11}, c_{11}\right) e_{21}^{(1)}\right) \\
= & \left(\left(a_{11}, b_{11}, c_{11}\right) e_{12}^{(1)}\right) e_{21}^{(1)} \\
= & 0 .
\end{aligned}
$$

Portanto, $\mathcal{A}_{11}$ é um anel associativo.

Finalmente, vamos a mostrar a comutatividade de $\mathcal{A}_{11}$. Por (2.7) sabemos que

$$
e_{21}^{(2)} e_{21}^{(3)}=(1-2) e_{12}^{(1)}=-e_{12}^{(1)} \quad \text { e } \quad e_{21}^{(2)} e_{21}^{(3)}+e_{21}^{(3)} e_{21}^{(2)}=e_{21}^{(2)} \circ e_{21}^{(3)}=0,
$$

os quais implicam, $e_{12}^{(1)}=e_{21}^{(3)} e_{21}^{(2)}$. Então, por $(2.11)$

$$
\begin{aligned}
{\left[a_{11}, b_{11}\right] e_{12}^{(1)}=} & \left(a_{11} b_{11}\right)\left(e_{21}^{(3)} e_{21}^{(2)}\right)-\left(b_{11} a_{11}\right)\left(e_{21}^{(3)} e_{21}^{(2)}\right) \\
= & -\left(e_{21}^{(2)} b_{11}\right)\left(e_{21}^{(3)} a_{11}\right)+\left[e_{21}^{(2)}\left(b_{11} e_{21}^{(3)}\right)\right] a_{11}+\left[a_{11}\left(b_{11} e_{21}^{(3)}\right)\right] e_{21}^{(2)} \\
& +\left(e_{21}^{(2)} a_{11}\right)\left(e_{21}^{(3)} b_{11}\right)-\left[e_{21}^{(2)}\left(a_{11} e_{21}^{(3)}\right)\right] b_{11}-\left[b_{11}\left(a_{11} e_{21}^{(3)}\right)\right] e_{21}^{(2)} \\
= & -\left(e_{21}^{(2)} b_{11}\right)\left(e_{21}^{(3)} a_{11}\right)+\left(e_{21}^{(2)} a_{11}\right)\left(e_{21}^{(3)} b_{11}\right) .
\end{aligned}
$$

Assim, novamente por (2.11)

$$
\begin{aligned}
\left(e_{21}^{(2)} b_{11}\right)\left(e_{21}^{(3)} a_{11}\right) & =-\left(a_{11} b_{11}\right)\left(e_{21}^{(3)} e_{21}^{(2)}\right)+\left[a_{11}\left(b_{11} e_{21}^{(3)}\right)\right] e_{21}^{(2)}+\left[e_{21}^{(2)}\left(b_{11} e_{21}^{(3)}\right)\right] a_{11} \\
& =-\left(a_{11} b_{11}\right)\left(e_{21}^{(3)} e_{21}^{(2)}\right) \\
& =\left(a_{11} b_{11}, e_{21}^{(3)}, e_{21}^{(2)}\right)-\left(\left(a_{11} b_{11}\right) e_{21}^{(3)}\right) e_{21}^{(2)} \\
& =\left(e_{21}^{(2)}, a_{11} b_{11}, e_{21}^{(3)}\right)
\end{aligned}
$$

e de forma semelhante $\left(e_{21}^{(2)} a_{11}\right)\left(e_{21}^{(3)} b_{11}\right)=\left(e_{21}^{(2)}, b_{11} a_{11}, e_{21}^{(3)}\right)$. Mas, lembrando que $e_{21}^{(2)} e_{21}^{(3)}=$ $-e_{12}^{(1)}$ e usando a seguinte identidade obtida de $(2.13) x[y(z w)]=-x[w(z y)]+[(x y) z] w+$ $[(x w) z] y$, obtemos

$$
\begin{aligned}
\left(e_{21}^{(2)}, b_{11} a_{11}, e_{21}^{(3)}\right) & =-\left(e_{21}^{(2)}, e_{21}^{(3)}, b_{11} a_{11}\right) \\
& =-\left(e_{21}^{(2)} e_{21}^{(3)}\right)\left(b_{11} a_{11}\right)+e_{21}^{(2)}\left(e_{21}^{(3)}\left(b_{11} a_{11}\right)\right) \\
& =-e_{21}^{(2)}\left[a_{11}\left(b_{11} e_{21}^{(3)}\right)\right]+\left[\left(e_{21}^{(2)} e_{21}^{(3)}\right) b_{11}\right] a_{11}+\left[\left(e_{21}^{(2)} a_{11}\right) b_{11}\right] e_{21}^{(3)} \\
& =\left(e_{21}^{(2)}, a_{11} b_{11}, e_{21}^{(3)}\right),
\end{aligned}
$$

por conseguinte, $\left(e_{21}^{(2)} a_{11}\right)\left(e_{21}^{(3)} b_{11}\right)=\left(e_{21}^{(2)} b_{11}\right)\left(e_{21}^{(3)} a_{11}\right)$. Portanto,

$$
\left[a_{11}, b_{11}\right] e_{12}^{(1)}=0 .
$$


Logo, por (2.6)

$$
\begin{aligned}
{\left[a_{11}, b_{11}\right]=\left[a_{11}, b_{11}\right] e_{11}=} & {\left[a_{11}, b_{11}\right]\left(e_{12}^{(1)} e_{21}^{(1)}\right) } \\
\stackrel{(2.2)}{=} & \left(\left[a_{11}, b_{11}\right] \circ e_{12}^{(1)}\right) e_{21}^{(1)}-e_{12}^{(1)}\left(\left[a_{11}, b_{11}\right] e_{21}^{(1)}\right) \\
= & \left(\left[a_{11}, b_{11}\right] e_{12}^{(1)}\right) e_{21}^{(1)} \\
= & 0 ;
\end{aligned}
$$

assim, $\left[a_{11}, b_{11}\right]=0$. Portanto, $\mathcal{A}_{11}$ é um anel associativo e comutativo.

Isto prova o Lema.

Q.E.D.

Como no Lema 3, usando o mesmo processo de demonstração pode-se mostrar que $\mathcal{A}_{22}$ é uma subálgebra associativa e comutativa de $\mathcal{A}$.

Definimos as "funções de coordenadas" $\pi_{i j}^{(k)}: \mathcal{A} \longrightarrow \Omega$ sobre $\mathcal{A}$ tomando $\pi_{i j}^{(k)}$ igual a zero em todas as componentes exceto em $\mathcal{A}_{i j}^{(k)}$, e sobre $\mathcal{A}_{i j}^{(k)}$ estão definidas da seguinte forma:

$$
\begin{gathered}
\pi_{11}\left(a_{11}\right)=a_{11}, \quad \pi_{22}\left(a_{22}\right)=e_{12}\left(a_{22} e_{21}\right)=L_{e_{12}} R_{e_{21}}\left(a_{22}\right), \\
\pi_{12}^{(k)}\left(a_{12}^{(k)}\right)=a_{12}^{(k)} e_{21}^{(k)}=R_{e_{21}^{(k)}}\left(a_{12}^{(k)}\right), \quad \pi_{21}^{(k)}\left(a_{21}^{(k)}\right)=e_{12}^{(k)} a_{21}^{(k)}=L_{e_{12}^{(k)}}\left(a_{21}^{(k)}\right),
\end{gathered}
$$

onde pode-se observar que $\pi_{22}=L_{e_{12}} R_{e_{21}}, \pi_{12}^{(k)}=R_{e_{21}^{(k)}}$ e $\pi_{21}^{(k)}=L_{e_{12}^{(k)}}$. Além disso, definimos uma aplicação linear $\pi: \mathcal{A} \longrightarrow C(\Omega)$ dada por

$$
\pi(a)=\sum_{i=1}^{2} \pi_{i i}(a) e_{i i}+\sum_{k=1}^{3}\left(\pi_{12}^{(k)}(a) e_{12}^{(k)}+\pi_{21}^{(k)}(a) e_{21}^{(k)}\right) .
$$

Lema 4 A aplicação $\pi$ é um homomorfismo de álgebras, isto é, $\pi(a b)=\pi(a) \pi(b)$.

Demonstração: Primeiro vamos mostrar a igualdade $e_{12}\left(a_{22} e_{21}\right)=e_{12}^{(k)}\left(a_{22} e_{21}^{(k)}\right), k=2,3$. Isto segue das relações dos espaços de Peirce do Lema 1 ou 2 e, por (2.11)

$$
\begin{aligned}
& e_{12}^{(2)}\left(a_{22} e_{21}^{(2)}\right) \stackrel{(2.7)}{=}\left(e_{21}^{(1)} e_{21}^{(3)}\right)\left(a_{22} e_{21}^{(2)}\right) \\
& =-\left(e_{21}^{(2)} e_{21}^{(3)}\right)\left(a_{22} e_{21}^{(1)}\right)+\left[e_{21}^{(1)}\left(e_{21}^{(3)} a_{22}\right)\right] e_{21}^{(2)}+\left[e_{21}^{(2)}\left(e_{21}^{(3)} a_{22}\right)\right] e_{21}^{(1)} \\
& =-\left(-e_{12}^{(1)}\right)\left(a_{22} e_{21}^{(1)}\right) \\
& =e_{12}^{(1)}\left(a_{22} e_{21}^{(1)}\right) \text {, } \\
& e_{12}^{(3)}\left(a_{22} e_{21}^{(2)}\right) \stackrel{(2.7)}{=}-\left(e_{21}^{(1)} e_{21}^{(2)}\right)\left(a_{22} e_{21}^{(3)}\right) \\
& =-\left(e_{21}^{(3)} e_{21}^{(2)}\right)\left(a_{22} e_{21}^{(1)}\right)+\left[e_{21}^{(1)}\left(e_{21}^{(2)} a_{22}\right)\right] e_{21}^{(3)}+\left[e_{21}^{(3)}\left(e_{21}^{(2)} a_{22}\right)\right] e_{21}^{(1)} \\
& =-\left(-e_{12}^{(1)}\right)\left(a_{22} e_{21}^{(1)}\right) \\
& =e_{12}^{(1)}\left(a_{22} e_{21}^{(1)}\right) \text {. }
\end{aligned}
$$

Para provar que $\pi$ é um homomorfismo de álgebras, precisaremos as seguintes expressões das funções de coordenadas $\pi_{i j}^{(k)}$ : por $(2.2)$ e $\left(e_{i j}^{(k)}, a_{j j}, e_{j i}^{(k)}\right)=0$ para $i, j \neq$

$$
\pi_{12}^{(k)}\left(a_{11} e_{12}^{(k)}\right)=\left(a_{11} e_{12}^{(k)}\right) e_{21}^{(k)}=a_{11}\left(e_{12}^{(k)} \circ e_{21}^{(k)}\right)-\left(a_{11} e_{21}^{(k)}\right) e_{12}^{(k)}=a_{11},
$$




$$
\begin{gathered}
\pi_{12}^{(k)}\left(e_{12}^{(k)} a_{22}\right)=\left(e_{12}^{(k)} a_{22}\right) e_{21}^{(k)}=e_{12}^{(k)}\left(a_{22} e_{21}^{(k)}\right)=e_{12}\left(a_{22} e_{21}\right)=\pi_{22}\left(a_{22}\right), \\
\pi_{21}^{(k)}\left(e_{21}^{(k)} a_{11}\right)=e_{12}^{(k)}\left(e_{21}^{(k)} a_{11}\right)=\left(e_{12}^{(k)} \circ e_{21}^{(k)}\right) a_{11}-e_{21}^{(k)}\left(e_{12}^{(k)} a_{11}\right)=a_{11}, \\
\pi_{21}^{(k)}\left(a_{22} e_{21}^{(k)}\right)=e_{12}^{(k)}\left(a_{22} e_{21}^{(k)}\right)=e_{12}\left(a_{22} e_{21}\right)=\pi_{22}\left(a_{22}\right),
\end{gathered}
$$

Basta analisar os casos quando $a$ e $b$ estão nos espaços de Peirce $\mathcal{A}_{i j}^{(k)}$, assim:

(A) $\pi_{i i}(a b)=\pi_{i i}(a) \pi_{i i}(b) ; a, b \in \mathcal{A}_{i i}$.

(B) $\pi_{i j}^{(k)}(a b)=\pi_{i i}(a) \pi_{i j}^{(k)}(b) ; a \in \mathcal{A}_{i i}$ e $b \in \mathcal{A}_{i j}^{(k)}$

(C) $\pi_{i j}^{(k)}(a b)=\pi_{i j}^{(k)}(a) \pi_{j j}(b) ; a \in \mathcal{A}_{i j}^{(k)}$ e $b \in \mathcal{A}_{j j}$

(D) $\pi_{i i}(a b)=\pi_{i j}^{(k)}(a) \pi_{j i}^{(k)}(b) ; a \in \mathcal{A}_{i j}^{(k)}$ e $b \in \mathcal{A}_{j i}^{(k)}$

(E) $\pi_{j i}^{(k+2)}(a b)=\delta_{i j} \pi_{i j}^{(k)}(a) \pi_{i j}^{(k+1)}(b) ; a \in \mathcal{A}_{i j}^{(k)}$ e $b \in \mathcal{A}_{i j}^{(k+1)}$

onde todas as igualdades são resultado das relações de ortogonalidade do Lema 2. Usando os Lemas 1 e 2 para expressar as componente $\mathcal{A}_{i j}^{(k)}$ e, junto com as expressões de acima das funções de coordenadas $\pi_{i j}^{(k)}$, obtemos os seguintes casos:

(A1) Sejam $a_{11}, b_{11} \in \mathcal{A}_{11}$, então $\pi_{11}\left(a_{11} b_{11}\right)=a_{11} b_{11}=\pi_{11}\left(a_{11}\right) \pi_{11}\left(b_{11}\right)$.

(B1) Considere $a_{11} \in \mathcal{A}_{11}$ e $b_{11} e_{12}^{(k)}=b_{12}^{(k)} \in \mathcal{A}_{12}^{(k)}$

$$
\pi_{12}^{(k)}\left(a_{11} b_{12}^{(k)}\right)=\pi_{12}^{(k)}\left(a_{11}\left(b_{11} e_{12}^{(k)}\right)\right)=\pi_{12}^{(k)}\left(\left(a_{11} b_{11}\right) e_{12}^{(k)}\right)=a_{11} b_{11}=\pi\left(a_{11}\right) \pi_{12}^{(k)}\left(b_{12}^{(k)}\right) .
$$

(C1) Agora, sejam $e_{21}^{(k)} a_{11}=a_{21}^{(k)} \in \mathcal{A}_{21}^{(k)}$ e $b_{11} \in \mathcal{A}_{11}$

$$
\pi_{21}^{(k)}\left(a_{21}^{(k)} b_{11}\right)=\pi_{21}^{(k)}\left(\left(e_{21}^{(k)} a_{11}\right) b_{11}\right)=\pi_{12}^{(k)}\left(e_{21}^{(k)}\left(a_{11} b_{11}\right)\right)=a_{11} b_{11}=\pi_{21}^{(k)}\left(a_{21}^{(k)}\right) \pi_{11}\left(b_{11}\right)
$$

(D1) Tome $a_{11} e_{12}^{(k)}=a_{12}^{(k)} \in \mathcal{A}_{12}^{(k)}$ e $e_{21}^{(k)} b_{11}=b_{21}^{(k)} \in \mathcal{A}_{21}^{(k)}$, logo

$$
\left.a_{12}^{(k)} b_{21}^{(k)}=\left(a_{11} e_{12}^{(k)}\right) b_{21}^{(k)} \stackrel{(2)}{=} a_{11}\left(e_{12}^{(k)} \circ b_{21}^{(k)}\right)-\left(a_{11} b_{21}^{(k)}\right) e_{12}^{(k)}\right)=a_{11}\left[e_{12}^{(k)}\left(e_{21}^{(k)} b_{11}\right)\right]=a_{11} b_{11} .
$$

Então,

$$
\pi_{11}\left(a_{12}^{(k)} b_{21}^{(k)}\right)=\pi_{11}\left(a_{11} b_{11}\right)=a_{11} b_{11}=\pi_{12}^{(k)}\left(a_{11} e_{12}^{(k)}\right) \pi_{21}^{(k)}\left(e_{21}^{(k)} b_{11}\right)=\pi_{12}^{(k)}\left(a_{12}^{(k)}\right) \pi_{21}^{(k)}\left(b_{21}^{(k)}\right) .
$$

(E1) Sejam $a_{11} e_{12}^{(k)}=a_{12}^{(k)} \in \mathcal{A}_{12}^{(k)}$ e $b_{11} e_{12}^{(k+1)}=b_{12}^{(k+1)} \in \mathcal{A}_{12}^{(k+1)}$, logo pela comutatividade de $\mathcal{A}_{11}$

$$
a_{12}^{(k)} b_{12}^{(k+1)}=\left(a_{11} e_{12}^{(k)}\right)\left(b_{11} e_{12}^{(k+1)}\right) \stackrel{(11)}{=}-\left(e_{12}^{(k+1)} e_{12}^{(k)}\right)\left(b_{11} a_{11}\right)=e_{21}^{(k+2)}\left(a_{11} b_{11}\right) .
$$

Portanto,

$$
\pi_{21}^{(k+2)}\left(a_{12}^{(k)} b_{12}^{(k+1)}\right)=\pi_{21}^{(k+2)}\left(e_{21}^{(k+2)}\left(a_{11} b_{11}\right)\right)=a_{11} b_{11}=\pi_{12}^{(k)}\left(a_{12}^{(k)}\right) \pi_{12}^{(k+1)}\left(b_{12}^{(k+1)}\right) .
$$

E analogamente, também temos:

(A2) Sejam $a_{22}, b_{22} \in \mathcal{A}_{22}$, então $\pi_{22}\left(a_{22} b_{22}\right)=a_{22} b_{22}=\pi_{22}\left(a_{22}\right) \pi_{22}\left(b_{22}\right)$. 
(B2) Considere $a_{22} \in \mathcal{A}_{22}$ e $b_{22} e_{21}^{(k)}=b_{21}^{(k)} \in \mathcal{A}_{21}^{(k)}$

$$
\pi_{21}^{(k)}\left(a_{22} b_{21}^{(k)}\right)=\pi_{21}^{(k)}\left(a_{22}\left(b_{22} e_{21}^{(k)}\right)\right)=\pi_{21}^{(k)}\left(\left(a_{22} b_{22}\right) e_{21}^{(k)}\right)=\pi_{22}\left(a_{22} b_{22}\right)=\pi_{22}\left(a_{22}\right) \pi_{21}^{(k)}\left(b_{21}^{(k)}\right) .
$$

(C2) Agora, sejam $e_{12}^{(k)} a_{22}=a_{12}^{(k)} \in \mathcal{A}_{12}^{(k)}$ e $b_{22} \in \mathcal{A}_{22}$

$$
\pi_{12}^{(k)}\left(a_{12}^{(k)} b_{22}\right)=\pi_{12}^{(k)}\left(\left(e_{12}^{(k)} a_{22}\right) b_{22}\right)=\pi_{12}^{(k)}\left(e_{12}^{(k)}\left(a_{22} b_{22}\right)\right)=\pi_{22}\left(a_{22} b_{22}\right)=\pi_{12}^{(k)}\left(a_{12}^{(k)}\right) \pi_{22}\left(b_{22}\right) .
$$

(D2) Tome $a_{22} e_{21}^{(k)}=a_{21}^{(k)} \in \mathcal{A}_{21}^{(k)}$ e $e_{12}^{(k)} b_{22}=b_{12}^{(k)} \in \mathcal{A}_{12}^{(k)}$, logo

$$
a_{21}^{(k)} b_{12}^{(k)}=\left(a_{22} e_{21}^{(k)}\right) b_{12}^{(k)} \stackrel{(2)}{=} a_{22}\left(e_{21}^{(k)} \circ b_{12}^{(k)}\right)-\left(a_{22} b_{12}^{(k)}\right) e_{21}^{(k)}=a_{22}\left[e_{21}^{(k)}\left(e_{12}^{(k)} b_{22}\right)\right]=a_{22} b_{22} .
$$

Então,

$$
\pi_{22}\left(a_{21}^{(k)} b_{12}^{(k)}\right)=\pi_{22}\left(a_{22} b_{22}\right)=\pi_{22}\left(a_{22}\right) \pi_{22}\left(b_{22}\right)=\pi_{21}^{(k)}\left(a_{21}^{(k)}\right) \pi_{12}^{(k)}\left(b_{12}^{(k)}\right)
$$

(E2) Sejam $a_{22} e_{21}^{(k)}=a_{21}^{(k)} \in \mathcal{A}_{21}^{(k)}$ e $b_{22} e_{21}^{(k+1)}=b_{21}^{(k+1)} \in \mathcal{A}_{21}^{(k+1)}$, logo pela comutatividade de $\mathcal{A}_{22}$

$$
a_{21}^{(k)} b_{21}^{(k+1)}=\left(a_{22} e_{21}^{(k)}\right)\left(b_{22} e_{21}^{(k+1)}\right) \stackrel{(11)}{=}\left(e_{21}^{(k+1)} e_{21}^{(k)}\right)\left(b_{22} a_{22}\right)=-e_{12}^{(k+2)}\left(a_{22} b_{22}\right)
$$

Portanto,

$$
\pi_{12}^{(k+2)}\left(a_{21}^{(k)} b_{21}^{(k+1)}\right)=\pi_{12}^{(k+2)}\left(-e_{12}^{(k+2)}\left(a_{22} b_{22}\right)\right)=-\pi_{22}\left(a_{22} b_{22}\right)=-\pi_{21}^{(k)}\left(a_{21}^{(k)}\right) \pi_{21}^{(k+1)}\left(b_{21}^{(k+1)}\right) .
$$

Isso prova o Lema.

\section{Q.E.D.}

O Teorema de coordenatização de Wedderburn diz que uma álgebra associativa com elemento identidade contendo matrizes unitárias é uma álgebra matricial. Da mesma forma, o Teorema de coordenatização de Zorn diz que uma álgebra alternativa com elemento identidade contendo matrizes unitárias de Cayley-Dickson é uma álgebra matricial de CayleyDickson.

Teorema 7 (Teorema de coordenatização de Zorn) Seja $\mathcal{A}$ uma álgebra alternativa com elemento identidade 1 que contém um sistema de matrizes unitárias de Cayley-Dickson tal que $e_{11}+e_{22}=1$. Então, $\mathcal{A}$ é uma álgebra matricial de Cayley-Dickson sobre o anel $\Omega=\mathcal{A}_{11}$, isto é, $\mathcal{A} \cong C(\Omega)$.

Demonstração: Consideremos a aplicação $\pi: \mathcal{A} \longrightarrow C(\Omega)$ dada acima $\pi(a)=\sum_{i=1}^{2} \pi_{i i}(a) e_{i i}+$ $\sum_{k=1}^{3}\left(\pi_{12}^{(k)}(a) e_{12}^{(k)}+\pi_{21}^{(k)}(a) e_{21}^{(k)}\right)$ com $a=a_{11}+\sum_{k=1}^{3} a_{12}^{(k)}+\sum_{k=1}^{3} a_{21}^{(k)}+a_{22}$ dada pela decomposição de $\mathcal{A}$ nos Lemas 1 e 2 .

Pelo Lema 4, já sabemos que $\pi$ é um homomorfismo de álgebras. Portanto, será suficiente mostrar que $\pi$ é bijetiva.

Usando as expressões das funções de coordenadas do Lema 4, todas as aplicações $\pi_{i j}^{(k)}$ são bijeções; é claro que $\pi_{11}$ é uma bijeção, $\pi_{12}^{(k)}=R_{e_{21}^{(k)}}$ é a inversa de

$$
R_{e_{12}^{(k)}}: \mathcal{A}_{11} \rightarrow \mathcal{A}_{11} e_{12}^{(k)}=\mathcal{A}_{12}^{(k)}
$$


$\pi_{21}^{(k)}=L_{e_{12}^{(k)}}$ é a inversa de $L_{e_{21}^{(k)}}: \mathcal{A}_{11} \rightarrow e_{21}^{(k)} \mathcal{A}_{11}=\mathcal{A}_{21}^{(k)}$, enquanto $\pi_{22}=L_{e_{12}^{(k)}} R_{e_{21}^{(k)}}$ tem inversa $L_{e_{21}^{(k)}} R_{e_{12}^{(k)}}$. Assim, isto mostra que $\pi$ é um homomorfismo bijetivo. Portanto, $\mathcal{A} \cong C(\Omega)$.

Isso prova o Teorema.

Q.E.D.

Ao considerar uma álgebra associativa e comutativa $\Omega$ com elemento identidade podemos obter do Teorema 7 a seguinte equivalência de categorias.

Corolário 8 A categoria dos bimódulos alternativos unitários sobre $C(\Omega)$ é equivalente à categoria dos módulos associativos comutativos unitários sobre $\Omega$.

Demonstração: Vamos denotar por $\operatorname{Mod}_{A s s C o m} \Omega$ a categoria dos módulos associativos e comutativos sobre $\Omega$, e por $\operatorname{Bimod}_{A l t} C(\Omega)$ a categoria dos bimódulos alternativos sobre $C(\Omega)$. Então, desejamos mostrar que

$$
\operatorname{Mod}_{A s s C o m} \Omega \simeq \operatorname{Bimod}_{A l t} C(\Omega) .
$$

Seja $N \in \operatorname{Obj}\left(\operatorname{Mod}_{A s s C o m} \Omega\right)$ e considere a extensão cisão nula $E=\Omega \oplus N$ de $\Omega$ por $N, E$ é uma álgebra associativa comutativa. Assim, podemos formar a álgebra matricial de Cayley-Dickson $K=C(E)$, que contém a álgebra $C(\Omega)$ como uma subálgebra. Também, $K$ contém o ideal $M=N_{2} \cap K=C(N)$, que é o conjunto de matrizes de $K$ cujas entradas estão no ideal $N$ de $E$. Então $M$ é um bimódulo alternativo unitário para $C(\Omega)$, relativo à multiplicação definida em $K$. Vamos chamar ao $M$ o $C(\Omega)$-bimódulo associado com o dado módulo $N$ de $\Omega$ e denote

$$
M=\mathcal{G}(N) .
$$

Como $E=\Omega \oplus N$ temos, $K=C(\Omega) \oplus M$. Também, $N^{2}=0$ em $E$ implica que $M^{2}=0$ em $K, \operatorname{assim} K$ é a extensão cisão nula de $C(\Omega)$ por seu bimódulo $M$.

Pode-se verificar facilmente que $\mathcal{G}$ é realmente um funtor da categoria $\operatorname{Mod}_{\text {AssCom }} \Omega$ na categoria $\operatorname{Bimod}_{A l t} C(\Omega)$. Além disso, para cada par de objetos $N$ e $N^{\prime} \operatorname{de} \operatorname{Mod}_{A s s C o m} \Omega$, a seguinte igualdade é válida:

$$
\mathcal{G}\left(\operatorname{Hom}\left(N, N^{\prime}\right)\right)=\operatorname{Hom}\left(\mathcal{G}(N), \mathcal{G}\left(N^{\prime}\right)\right) \text {. }
$$

Portanto, $N$ e $N^{\prime}$ são isomorfos, se e somente se, $\mathcal{G}(N)$ e $\mathcal{G}\left(N^{\prime}\right)$ são isomorfos.

Similarmente, o funtor $\mathcal{G}$ oferece um isomorfismo reticulado do reticulado dos submódulos de $N$, relativo a $\Omega$ sobre o reticulado dos sub-bimódulos de $M$ sobre $C(\Omega)$.

Para completar a nossa redução da teoria dos bimódulos para $C(\Omega)$ ao dos módulos para $\Omega$, agora mostraremos que cada $C(\Omega)$-bimódulo unitário é isomorfo a algum bimódulo associado com um módulo unitário para $\Omega$.

Considere um bimódulo alternativo unitário $V$ para $C(\Omega)$, e seja

$$
\mathcal{A}=C(\Omega) \oplus V
$$

a extensão cisão nula de $C(\Omega)$ por $V$. Assim, $\mathcal{A}$ é uma álgebra alternativa que contém a álgebra matricial de Cayley-Dickson $C(\Omega)$ como uma subálgebra com o mesmo elemento identidade, então pelo Teorema de Coordenatização de Zorn - Teorema 7 - existe um álgebra associativa e comutativa $D$ tal que $\mathcal{A}=C(D)$. Considere o conjunto $W$ dos elementos de $D$ que aparecem nas entradas das matrizes de $V$. Logo,

$$
V:=C(W)
$$


onde $W \triangleleft D$ e $W^{2}=0$ em $D$ pois, $V \triangleleft \mathcal{A}$ e $V^{2}=0$ em $\mathcal{A}$, e assim, $D=\Omega \oplus N$. Logo, $D$ é a extensão cisão nula de $\Omega$ por seu módulo $W$, então $W$ é um módulo associativo e comutativo unitário sobre $\Omega$. Portanto $\mathcal{G}(W)=V$.

Isto prova o Corolário.

Q.E.D.

Da equivalência de categorias dada no Corolário 8, podemos classificar aos bimódulos alternativos irredutíveis unitários sobre $C(F)$, dada no Corolário 9, mas nós damos uma demonstração desse resultado usando diretamente o Teorema de Coordenatização de Zorn.

Corolário 9 Cada bimódulo alternativo irredutível unitário sobre a álgebra matricial de Cayley-Dickson $C(F)$, é isomorfo ao bimódulo regular $\operatorname{Reg}(C(F))$.

Demonstração: Seja $V$ um bimódulo alternativo irredutível unitário sobre $C(F)$. Então, a extensão cisão nula $E=C(F) \oplus V$ de $C(F)$ pelo bimódulo $V$, é uma álgebra alternativa com a mesmo elemento identidade de $C(F)$. Portanto, pelo Teorema de Coordenatização de Zorn (Teorema 7), existe uma certa subálgebra $\Omega$ de $E$ tal que $E=C(\Omega)$.

A partir de $E=C(\Omega)$, considere o conjunto $D$ dos elementos de $\Omega$ que aparecem nas entradas das matrizes de $V$. Logo,

$$
V:=C(D)
$$

onde $D \triangleleft \Omega$ e $D^{2}=0$ pois, $V \triangleleft E$ e $V^{2}=0$ em $E$, e assim, $\Omega=F \cdot 1+D$ é a extensão cisão nula de $F \cdot 1$ por $D$. Mas, $D$ é um $F$-bimódulo associativo irredutível pois, $V$ o é. Portanto, $D=F \cdot 1$, o qual implica, $V=\operatorname{Reg}(C(F))$.

Isto prova o Corolário.

Q.E.D.

\subsection{Generalização de um teorema de Nathan Jacobson}

A decomposição de Peirce de uma álgebra alternativa foi dada de maneira geral no Lema 1 para alguns idempotentes especiais. Mas para não perder a ilação das nossas ideias aqui, vamos descrever o que é a decomposição de Peirce associada a um idempotente e logo depois, a decomposição de Peirce associada a um sistema de idempotentes.

Seja $\mathcal{A}$ uma álgebra alternativa com um elemento idempotente $e\left(e^{2}=e\right)$. Utilizando as equivalências de (2.1) em termos das multiplicações à direita e esquerda, temos que $R_{e}$ e $L_{e}$ são operadores lineares idempotentes sobre $\mathcal{A}$ que comutam. Logo, se segue que $\mathcal{A}$ é a soma direta de espaços vetoriais

$$
\mathcal{A}=\mathcal{A}_{11} \oplus \mathcal{A}_{10} \oplus \mathcal{A}_{01} \oplus \mathcal{A}_{00}
$$

onde $\mathcal{A}_{i j}(i, j=0,1)$ é o subespaço de $\mathcal{A}$ definido por

$$
\mathcal{A}_{i j}=\left\{x_{i j} \mid e x_{i j}=i x_{i j}, x_{i j} e=j x_{i j}\right\}, \quad i, j=0,1
$$

com as relações dadas no Lema 1, os quais são

$$
\begin{gathered}
\mathcal{A}_{i i}^{2} \subseteq \mathcal{A}_{i i}, \quad \mathcal{A}_{i i} \mathcal{A}_{j j}=0 \\
\mathcal{A}_{i i} \mathcal{A}_{i j}+\mathcal{A}_{i j} \mathcal{A}_{j j} \subseteq \mathcal{A}_{i j}, \quad \mathcal{A}_{j j} \mathcal{A}_{i j}=\mathcal{A}_{i j} \mathcal{A}_{i i}=0, \\
\mathcal{A}_{i j} \mathcal{A}_{i j} \subseteq \mathcal{A}_{j i}, \quad \mathcal{A}_{i j} \mathcal{A}_{j i} \subseteq \mathcal{A}_{i i}
\end{gathered}
$$


onde $i \neq j$.

Os idempotentes $e_{1}, e_{2}, \ldots, e_{n}$ em uma álgebra $\mathcal{A}$ (arbitrária) são chamados sistema de idempotentes ortogonais quando $e_{i} e_{j}=0$ para $i \neq j$. Observe que qualquer soma $e=e_{1}+e_{2}+\ldots+e_{n}$ de um sistema de idempotentes ortogonais $(n \geq 1)$ é um idempotente. Além disso, $e e_{i}=e_{i} e=e_{i}(i=1,2, \ldots, n)$.

No Lema 1 tínhamos observado que a relação $\left(x, e_{11}, e_{22}\right)=0$ é válida em cada álgebra alternativa, onde $e_{11}$ e $e_{22}$ são elementos da base da álgebra de matrizes de Cayley-Dickson com $e_{11}+e_{22}=1$. Mas, na verdade, essa relação é válida em cada álgebra alternativa para um sistema de idempotentes ortogonais, isto é, $\left(x, e_{i}, e_{j}\right)=0$ para $i \neq j$ (veja, [12]).

Uma decomposição de Peirce mais refinada para uma álgebra alternativa $\mathcal{A}$ é a seguinte decomposição, relacionada a um sistema de idempotentes ortogonais $e_{1}, \ldots, e_{n}$ em $\mathcal{A}$ : $\mathcal{A}$ é a soma direta de espaços vetoriais

$$
\mathcal{A}=\sum \mathcal{A}_{i j} \quad(i, j=1 \ldots, n),
$$

onde os $\mathcal{A}_{i j}$ são subespaços de $\mathcal{A}$ e estão definidos por

$$
\mathcal{A}_{i j}=\left\{x_{i j} \mid e_{k} x_{i j}=\delta_{k i} x_{i j}, x_{i j} e_{k}=\delta_{j k} x_{i j} \text { para } k=1, \ldots, n\right\} \quad(i, j=0,1, \ldots, n) .
$$

Os subespaços $\mathcal{A}_{i j}$ em (2.17), satisfazem também relações semelhantes aos subespaços de Peirce (2.15), obtidas por um idempotente:

$$
\begin{gathered}
\mathcal{A}_{i j} \mathcal{A}_{j k} \subseteq \mathcal{A}_{i k}, \quad(i, j, k=0,1, \ldots, n), \\
\mathcal{A}_{i j} \mathcal{A}_{i j} \subseteq \mathcal{A}_{j i}, \quad(i, j=0,1, \ldots, n), \\
\mathcal{A}_{i j} \mathcal{A}_{k l}=0, \quad j \neq k, \quad(i, j) \neq(k, l) \quad(i, j, k, l=0,1, \ldots, n), \\
\left(x_{i i}, y_{i j} z_{j i}, t_{i i}\right)=0 \text { se } i \neq j \text { para todo } x_{i i}, t_{i i} \in \mathcal{A}_{i i}, y_{i j} \in \mathcal{A}_{i j} \text { e } z_{j i} \in \mathcal{A}_{j i},
\end{gathered}
$$

e muitas outras que estão dadas no livro de Schafer [12].

Lema 5 Seja $\mathcal{A}$ uma álgebra alternativa que contém um sistema de idempotentes ortogonais $e_{1}, e_{2}, \ldots, e_{n}$. Seja (2.16) a decomposição de Peirce de $\mathcal{A}$ associada a $e_{1}, e_{2}, \ldots, e_{n}$. Então, a álgebra $\mathcal{A}$ é associativa, se e somente se, cada $\mathcal{A}_{i i}$ é associativa e $\mathcal{A}_{i j}^{2}=0$ para $i \neq j$. Neste caso, $\mathcal{A}_{i i}$ será associativa se $\mathcal{A}_{i i}=\mathcal{A}_{i j} \mathcal{A}_{j i}$ para alguns $i \neq j$, e $\mathcal{A}_{i j}^{2}=0$ se $\mathcal{A}_{i j}=\mathcal{A}_{i k} \mathcal{A}_{k j}$ para algum $k \neq i, j$.

Demonstração: Certamente as duas condições são necessárias. Também elas são suficientes pois, $\left(a_{i i}, b_{i i}, c_{i i}\right)=0$ se $\mathcal{A}_{i i}$ é associativa, $\left(a_{i j}, b_{i j}, \mathcal{A}\right)=0$ porque $\mathcal{A}_{i j}^{2}=0$, e através das propriedades da decomposição de Peirce para álgebras alternativas (2.18), (2.19) e (2.20); de modo que todos os produtos diferentes de zero devem ter índices ligados.

Usando a identidade

$$
(x y, z, w)=x(y, z, w)+(x, z, w) y-(x, y,[z, w])
$$

que é válida em toda álgebra alternativa. Assim, para todo $a_{i i}, b_{i i} \in \mathcal{A}_{i i}$ e $c_{i j} d_{j i} \in \mathcal{A}_{i j} \mathcal{A}_{j i}$

$$
\left(c_{i j} d_{j i}, a_{i i}, b_{i i}\right)=c_{i j}\left(d_{j i}, a_{i i}, b_{i i}\right)+\left(c_{i j}, a_{i i}, b_{i i}\right) d_{j i}-\left(c_{i j}, d_{j i},\left[a_{i i}, b_{i i}\right]\right)=0,
$$

isto significa que $\mathcal{A}_{i j} \mathcal{A}_{j i}$ se associa com $\mathcal{A}_{i i}$. Quando $\mathcal{A}_{i j} \mathcal{A}_{j i}=\mathcal{A}_{i i}$ a álgebra $\mathcal{A}_{i i}$ é associativa. Quando $\mathcal{A}_{i j}=\mathcal{A}_{i k} \mathcal{A}_{k j}$, então pela identidade linearizada da identidade de Moufang do meio 


$$
\begin{aligned}
\mathcal{A}_{i j}^{2}=\mathcal{A}_{i j} \mathcal{A}_{i j} & =\left(\mathcal{A}_{i k} \mathcal{A}_{k j}\right)\left(\mathcal{A}_{i k} \mathcal{A}_{k j}\right) \\
& \subseteq-\left(A_{k j} \mathcal{A}_{k j}\right)\left(\mathcal{A}_{i k} \mathcal{A}_{i k}\right)+\left(\mathcal{A}_{i k}\left(\mathcal{A}_{k j} \mathcal{A}_{i k}\right)\right) \mathcal{A}_{k j}+\left(\mathcal{A}_{k j}\left(\mathcal{A}_{k j} \mathcal{A}_{i k}\right)\right) \mathcal{A}_{k j} \\
\stackrel{(2.20)}{=} & (0)
\end{aligned}
$$

pois todos os espaços de Peirce são distintos se $i, j, k \neq$.

Isto prova o Lema.

Q.E.D.

Lema 6 Seja $\mathcal{A}$ uma álgebra alternativa com idempotente e $\neq 1$. Seja (2.14) a decomposição de Peirce de $\mathcal{A}$ associada a e. Suponha que existem elementos $a_{10}$ em $\mathcal{A}_{10}$ e $b_{01}$ em $\mathcal{A}_{01}$, tais que,

$$
a_{10} b_{01}=e
$$

Então, e $\mathcal{A} e=\mathcal{A}_{10} \mathcal{A}_{01}$ é associativa.

Demonstração: É claro que $e \mathcal{A} e=\mathcal{A}_{11}$. Também, $\mathcal{A}_{10} \mathcal{A}_{01} \subseteq \mathcal{A}_{11}$. Vamos mostrar a outra inclusão. Seja $a_{11}$ um elemento de $\mathcal{A}_{11}$, então

$$
a_{11}=a_{11} e=a_{11}\left(a_{10} a_{01}\right) \stackrel{(2.2)}{=}\left(a_{11} \circ a_{10}\right) a_{01}-a_{10}\left(a_{11} a_{01}\right)=\left(a_{11} a_{10}\right) a_{01} \in \mathcal{A}_{10} \mathcal{A}_{01}
$$

para alguns $a_{10} \in \mathcal{A}_{01}$ e $a_{01} \in \mathcal{A}_{01}$. Portanto $e \mathcal{A} e=\mathcal{A}_{11}=\mathcal{A}_{10} \mathcal{A}_{01}$. Também (2.21) implica que $\mathcal{A}_{10} \mathcal{A}_{01}$ é associativa.

Isto prova o Lema.

Q.E.D.

Agora, podemos enunciar e provar o teorema principal desta seção que é válido para as álgebras alternativas de dimensão e característica arbitrária.

Teorema 10 Seja $\mathcal{A}$ uma álgebra alternativa com elemento identidade 1 que contém um sistema de idempotentes ortogonais $e_{1}, e_{2}, \ldots, e_{n}$ tais que $1=e_{1}+e_{2}+\cdots+e_{n}$. Suponha que para cada $i \neq j$ existem $a_{i j} \in \mathcal{A}_{i j}$ e $b_{j i} \in \mathcal{A}_{j i}$, tais que,

$$
a_{i j} b_{j i}=e_{i} \quad \text { e } \quad b_{j i} a_{i j}=e_{j} .
$$

Se $n \geq 3$, então $\mathcal{A}$ é uma álgebra associativa.

Demonstração: Seja $\mathcal{A}=\sum \oplus \mathcal{A}_{i j}$ a decomposição de Peirce da álgebra alternativa $\mathcal{A}$ relativa aos idempotentes $e_{1}, e_{2}, \ldots, e_{n}$. Para qualquer $i=2,3, \ldots, n$, primeiro mostraremos que

$$
\mathcal{A}_{1 i}^{2}=\mathcal{A}_{i 1}^{2}=0 \quad(i=2, \ldots, n) .
$$

Considere $e=e_{1}+e_{i} \neq 1$ pois $n \geq 3$. Logo, pela hipótese, existem $a_{1 j} \in \mathcal{A}_{1 j}, a_{j 1} \in \mathcal{A}_{j 1}$, $a_{i k} \in \mathcal{A}_{i k}$ e $a_{k i} \in \mathcal{A}_{k i}(1, i, j, k \neq)$, tais que,

$$
e=e_{1}+e_{i}=a_{1 j} a_{j 1}+a_{i k} a_{k i}=\left(a_{1 j}+a_{i k}\right)\left(a_{j 1}+a_{k i}\right),
$$

onde os elementos $a_{1 j}+a_{i k}$ e $a_{j 1}+a_{k i}$ pertencem aos espaços de Peirce da decomposição de $\mathcal{A}$ associada a $e$. Assim, pelo Lema 6 , temos

$$
e \mathcal{A} e=e\left(\sum \oplus \mathcal{A}_{i j}\right) e=\left(e_{1}+e_{i}\right)\left(\sum \oplus \mathcal{A}_{i j}\right)\left(e_{1}+e_{i}\right)=\mathcal{A}_{11} \oplus \mathcal{A}_{1 i} \oplus \mathcal{A}_{i 1} \oplus \mathcal{A}_{i i}
$$


é associativa. Mais, essa decomposição é a decomposição de Peirce de $e \mathcal{A} e$ associada ao idempotente $e_{1}$; de fato, como

$$
e(e \mathcal{A} e)=e(e(\mathcal{A} e))=e^{2}(\mathcal{A} e)=e(\mathcal{A} e)=e \mathcal{A} e
$$

e

$$
(e \mathcal{A} e) e=((e \mathcal{A}) e) e=(e \mathcal{A}) e^{2}=(e \mathcal{A}) e=e \mathcal{A} e,
$$

então $e$ é o elemento identidade de $e \mathcal{A} e$ com a condição $e=e_{1}+e_{i}$. A decomposição de Peirce de $e \mathcal{A} e$ associada a $e_{1}$ está dada por

$$
e \mathcal{A} e=(e \mathcal{A} e)_{11} \oplus(e \mathcal{A} e)_{1 i} \oplus(e \mathcal{A} e)_{i 1} \oplus(e \mathcal{A} e)_{i i}
$$

Portanto,

$$
\begin{aligned}
(e \mathcal{A} e)_{11} & =e_{1}(e \mathcal{A} e) e_{1}=e_{1}\left(\mathcal{A}_{11} \oplus \mathcal{A}_{1 i} \oplus \mathcal{A}_{i 1} \oplus \mathcal{A}_{i i}\right) e_{1}=\mathcal{A}_{11}, \\
(e \mathcal{A} e)_{1 i} & =e_{1}(e \mathcal{A} e) e_{i}=e_{1}\left(\mathcal{A}_{11} \oplus \mathcal{A}_{1 i} \oplus \mathcal{A}_{i 1} \oplus \mathcal{A}_{i i}\right) e_{i}=\mathcal{A}_{1 i} \\
(e \mathcal{A} e)_{i 1} & =e_{i}(e \mathcal{A} e) e_{1}=e_{i}\left(\mathcal{A}_{11} \oplus \mathcal{A}_{1 i} \oplus \mathcal{A}_{i 1} \oplus \mathcal{A}_{i i}\right) e_{1}=\mathcal{A}_{i 1} \\
(e \mathcal{A} e)_{i i} & =e_{i}(e \mathcal{A} e) e_{i}=e_{i}\left(\mathcal{A}_{11} \oplus \mathcal{A}_{1 i} \oplus \mathcal{A}_{i 1} \oplus \mathcal{A}_{i i}\right) e_{i}=\mathcal{A}_{i i}
\end{aligned}
$$

e isso mostra o que queríamos. Portanto, pelo Lema $5, \mathcal{A}_{11}$ é associativa e $\mathcal{A}_{1 i}^{2}=\mathcal{A}_{i 1}^{2}=0$. Segue a partir da relação $\mathcal{A}_{i j} \mathcal{A}_{k l}=0$ para $j \neq k$ dada em $(2.20)$ que

$$
\left(\sum_{j=2}^{n} \mathcal{A}_{1 j}\right)^{2}=\left(\sum_{i=2}^{n} \mathcal{A}_{i 1}\right)^{2}=0 .
$$

Agora, consideramos a decomposição de Peirce $\mathcal{A}=\mathcal{A}_{11} \oplus \overline{\mathcal{A}}_{12} \oplus \overline{\mathcal{A}}_{21} \oplus \overline{\mathcal{A}}_{22}$ de $\mathcal{A}$ relativa ao idempotente $e_{1}$ (Não há ambiguidade sobre a notação $\mathcal{A}_{11}$, pois $\overline{\mathcal{A}}_{11}=\mathcal{A}_{11}$ ). Resulta do Lema 5 que $\mathcal{A}$ é associativa quando as componentes $\mathcal{A}_{11}$ e $\overline{\mathcal{A}}_{22}$ são associativas e $\overline{\mathcal{A}}_{12}^{2}=\overline{\mathcal{A}}_{21}^{2}=0$. Mas,

$$
\overline{\mathcal{A}}_{12}=\sum_{j=2}^{n} \mathcal{A}_{1 j}, \quad \overline{\mathcal{A}}_{21}=\sum_{i=2}^{n} \mathcal{A}_{i 1}, \quad \overline{\mathcal{A}}_{22}=\sum_{i, j=2}^{n} \mathcal{A}_{i j}
$$

onde,

$$
\mathcal{A}=\mathcal{A}_{11} \oplus \sum_{j=2}^{n} \mathcal{A}_{1 j} \oplus \sum_{i=2}^{n} \mathcal{A}_{i 1} \oplus \sum_{i, j=2}^{n} \mathcal{A}_{i j}
$$

é a decomposição de Peirce de $\mathcal{A}$ relativa aos idempotentes $e_{1}, e_{2}, \ldots, e_{n}$. Portanto, $\overline{\mathcal{A}}_{12}^{2}=$ $\overline{\mathcal{A}}_{21}^{2}=0$.

Já sabemos que $\mathcal{A}_{11}$ é associativa. Mas vamos mostrar esse resultado por meio de um argumento útil. Para fazer isso, primeiro provamos que $\left(a_{11}, b_{11}, c_{11}\right) a_{12}=0$ para quaisquer $a_{11}, b_{11}, c_{11} \in \mathcal{A}_{11}$ e $a_{12} \in \mathcal{A}_{12}$. De fato

$$
\begin{aligned}
\left(a_{11}, b_{11}, c_{11}\right) a_{12} & =\left(\left(a_{11} b_{11}\right) c_{11}\right) a_{12}-\left(a_{11}\left(b_{11} c_{11}\right)\right) a_{12} \\
\stackrel{(2.2)}{=} & \left(\left(a_{11} b_{11}\right) c_{11}\right) a_{12}-a_{11}\left(\left(b_{11} c_{11}\right) \circ a_{12}\right)+\left(a_{11} a_{12}\right)\left(b_{11} c_{11}\right) \\
= & \left(\left(a_{11} b_{11}\right) c_{11}\right) a_{12}-a_{11}\left(\left(b_{11} c_{11}\right) a_{12}\right)
\end{aligned}
$$

e logo usando a identidade linearizada de Moufang á direita (2.12), tem-se

$$
\left(\left(a_{11} b_{11}\right) c_{11}\right) a_{12}-a_{11}\left(\left(b_{11} c_{11}\right) a_{12}\right)=a_{11}\left(\left(a_{12} c_{11}\right) b_{11}\right)-\left(\left(a_{11} a_{12}\right) c_{11}\right) b_{11}=0
$$


pois $\mathcal{A}_{12} \mathcal{A}_{11}=0$ (isto se segue de (2.20)). Assim, como existem $a_{12} \in \mathcal{A}_{12}$ e $b_{21} \in \mathcal{A}_{21}$, tais que, $a_{12} b_{21}=e_{1}$ então, temos

$$
\begin{aligned}
\left(a_{11}, b_{11}, c_{11}\right) & =\left(a_{11}, b_{11}, c_{11}\right) e_{1} \\
& =\left(a_{11}, b_{11}, c_{11}\right)\left(a_{12} b_{21}\right) \\
& =-\left(\left(a_{11}, b_{11}, c_{11}\right), a_{12}, b_{21}\right)+\left(\left(a_{11}, b_{11}, c_{11}\right) a_{12}\right) b_{21} \\
& =\left(a_{12},\left(a_{11}, b_{11}, c_{11}\right), b_{21}\right) \\
& =0 .
\end{aligned}
$$

Portanto, $\mathcal{A}_{11}$ é uma álgebra associativa.

Usando os argumentos acima expostos com $e_{2}^{\prime}=e_{2}+e_{3}+\cdots+e_{n}$ e $e_{1}+e_{2}^{\prime}=1$. Vamos provar que $\overline{\mathcal{A}}_{22}$ é uma álgebra associativa. Temos que

$$
\overline{\mathcal{A}}_{22}=\left\{a \in \mathcal{A}: e_{2}^{\prime} a=a e_{2}^{\prime}=a\right\}
$$

e existem $a_{i 1} \in \mathcal{A}_{i 1}, b_{1 i} \in \mathcal{A}_{1 i}$ tais que $a_{i 1} b_{1 i}=e_{i}$ para $i=2,3, \ldots, n$. Logo, $e_{2}^{\prime}=a_{21} b_{12}+$ $a_{31} b_{13}+\cdots+a_{n 1} b_{1 n}$, então para quaisquer $a_{22}, b_{22}, c_{22} \in \overline{\mathcal{A}}_{22}$ tem-se

$$
\begin{aligned}
\left(a_{22}, b_{22}, c_{22}\right) & =\left(a_{22}, b_{22}, c_{22}\right) e_{2}^{\prime} \\
& =\left(a_{22}, b_{22}, c_{22}\right)\left(a_{21} b_{12}+a_{31} b_{13}+\cdots+a_{n 1} b_{1 n}\right) \\
& =\left(a_{22}, b_{22}, c_{22}\right)\left(a_{21} b_{12}\right)+\left(a_{22}, b_{22}, c_{22}\right)\left(a_{31} b_{13}\right)+\cdots+\left(a_{22}, b_{22}, c_{22}\right)\left(a_{n 1} b_{1 n}\right) .
\end{aligned}
$$

Logo, $\left(a_{22}, b_{22}, c_{22}\right)=0$ se, e somente se, cada termo da soma do lado direito da igualdade anterior é igual a zero. Assim,

$$
\begin{aligned}
\left(a_{22}, b_{22}, c_{22}\right) a_{21} & =\left(\left(a_{22} b_{22}\right) c_{22}\right) a_{21}-\left(a_{22}\left(b_{22} c_{22}\right)\right) a_{21} \\
\stackrel{(2.2)}{=} & \left(\left(a_{22} b_{22}\right) c_{22}\right) a_{21}-a_{22}\left(\left(b_{22} c_{22}\right) \circ a_{21}\right)+\left(a_{22} a_{21}\right)\left(b_{22} c_{22}\right) \\
= & \left(\left(a_{22} b_{22}\right) c_{22}\right) a_{21}-a_{22}\left(\left(b_{22} c_{22}\right) a_{21}\right)
\end{aligned}
$$

pois $\mathcal{A}_{21} \overline{\mathcal{A}}_{22}=0$ (isto se segue de (2.20)). Logo, usando a identidade linearizada de Moufang á direita (2.12), obtemos

$$
\left(\left(a_{22} b_{22}\right) c_{22}\right) a_{21}-a_{22}\left(\left(b_{22} c_{22}\right) a_{21}\right)=a_{22}\left(\left(a_{21} c_{22}\right) b_{22}\right)-\left(\left(a_{22} a_{21}\right) c_{22}\right) b_{22}=0
$$

Então,

$$
\left(a_{22}, b_{22}, c_{22}\right) a_{21}=0 .
$$

De forma análoga, $\left(a_{22}, b_{22}, c_{22}\right) a_{31}=\ldots=\left(a_{22}, b_{22}, c_{22}\right) a_{n 1}=0$. Portanto,

$$
\begin{aligned}
\left(a_{22}, b_{22}, c_{22}\right)\left(a_{21} b_{12}\right) & =-\left(\left(a_{22}, b_{22}, c_{22}\right), a_{21}, b_{12}\right)+\left(\left(a_{22}, b_{22}, c_{22}\right) a_{21}\right) b_{21} \\
& =\left(a_{21},\left(a_{22}, b_{22}, c_{22}\right), b_{12}\right) \\
& =0
\end{aligned}
$$

Da mesma forma, também temos $\left(a_{22}, b_{22}, c_{22}\right)\left(a_{31} b_{13}\right)=\ldots=\left(a_{22}, b_{22}, c_{22}\right)\left(a_{n 1} b_{1 n}\right)=0$. Logo, todos os termos da soma são iguais a 0 . Assim, $\left(a_{22}, b_{22}, c_{22}\right)=0$ para quaisquer $a_{22}, b_{22}, c_{22} \in \overline{\mathcal{A}}_{22}$, ou seja, $\overline{\mathcal{A}}_{22}$ é uma álgebra associativa. Portanto, $\mathcal{A}$ é uma álgebra associativa.

Isto prova o Teorema. 
Corolário 11 Seja $\mathcal{A}$ uma álgebra alternativa com elemento identidade, tal que, $\mathcal{A}$ contém $M_{n}(F)(n \geq 3)$ com o mesmo elemento identidade. Então, $\mathcal{A}$ é associativa, e portanto é válido o Teorema de Coordenatização de Wedderburn.

Usando o Corolário 11 podemos classificar os bimódulos alternativos unitários sobre $M_{n}(F), n \geq 3$.

Corolário 12 Cada bimódulo alternativo unitário para $M_{n}(F), n \geq 3$, é associativo.

Observação 13 Deve-se notar que o Corolário 12 foi também obtido por Jacobson usando sua classificação da redutibilidade completa dos bimódulos alternativos sobre corpos de característica diferente de 2 (veja [3], Teorema 11.1). Portanto, o Corolário 12 generaliza a aquele Teorema de Jacobson.

Observação 14 corolário 12 não é válido se $n=2$ pois $M_{2}(F)$ possui um bimódulo de Cayley irredutível unitário não-associativo de dimensão 2 (veja o exemplo 8 do Cap. 3). 


\section{Capítulo 3}

\section{O problema de Nathan Jacobson}

A teoria estrutural das álgebras alternativas de dimensão finita foi desenvolvida por Zorn: se $\mathcal{A}$ é uma álgebra altenativa de dimensão finita, então $\mathcal{A}$ contém um único ideal nilpotente maximal $R$ (o nil-radical da álgebra $\mathcal{A}$ ), e a álgebra quociente $\mathcal{A} / R$ é semissimples. Cada álgebra semissimples é uma soma direta de ideais que são álgebras simples. Cada álgebra alternativa simples é uma álgebra associativa o uma álgebra de Cayley-Dickson sobre seu centro (veja $[13,19])$.

A teoria das representações das álgebras alternativas foi iniciada nos artigos de R. D. Schafer [14] e N. Jacobson [3], onde foram estudadas as birepresentações das álgebras alternativas de dimensão finita. Em particular, em [14] foi mostrado que qualquer birepresentação de uma álgebra alternativa semissimples de característica 0 (sem nenhuma restrição sobre a dimensão) é completamente redutível, e em [3] foram classificados os bimódulos alternativos irredutíveis sobre as álgebras de dimensão finita.

N. Jacobson [3] descreveu os bimódulos alternativos irredutíveis sobre as álgebras dos quaterniões sob a suposição que o corpo base tem característica diferente de 2 e centraliza o bimódulo. I. Shestakov [16] estendeu esse resultado, retirando as restrições sobre a característica, a dimensão, e a condição da centralização.

O Teorema de Coordenatização de Wedderburn no caso $n \geq 3$ admite uma generalização formal para as álgebras alternativas, pois cada álgebra alternativa $\mathcal{A}$ que contém uma subálgebra $M_{n}(F)$ para $n \geq 3$ com o mesmo elemento identidade é associativa (veja o Corolário 11 do Cap. 2). O problema da descrição das álgebras alternativas que contém uma subálgebra $M_{2}(F)$ com o mesmo elemento identidade foi formulado por N. Jacobson em [2], o qual, vamos chamar "O problema de Nathan Jacobson". Neste capítulo, nós resolvemos esse problema sem nenhuma restrição sobre a dimensão e a característica do corpo $F$ (veja a secção 3.4).

Para obter a resposta do problema de Jacobson, usamos basicamente a descrição dos bimódulos alternativos unitários sobre os quaterniões, obtida por Shestakov [16]. E na parte final, desenvolvemos e usamos a teoria das álgebras alternativas tensoriais sobre uma fixada álgebra alternativa. Essa teoria foi desenvolvida por I. Kashuba, S. Ovsienko e I. Shestakov [5] para as álgebras de Jordan. Eles a usaram para classificar as álgebras de Jordan segundo seu tipo de representação. 


\section{1 Álgebras e bimódulos alternativos}

\subsection{1 Álgebras alternativas}

Ao longo deste capítulo, salvo indicação em contrário, $F$ denotará um corpo de característica arbitrária. Também, $\mathcal{A}$ denotará um espaço de dimensão arbitrária sobre $F$.

Como foi dito nos capítulos 2 e 3, uma álgebra alternativa (linear) é um espaço vetorial $\mathcal{A}$ com uma operação binária bilinear $(x, y) \mapsto x y$ satisfazendo as seguintes identidades:

$$
x^{2} y=x(x y), \quad(x y) y=x y^{2} .
$$

para todo $x, y \in \mathcal{A}$.

Usando o associador, as identidades (3.1) que definem às álgebras alternativas podem ser reescritas, como

$$
(x, x, y)=0, \quad(x, y, y)=0 .
$$

Lembremos que a primeira dessas é chamada de identidade alternativa à esquerda e a segunda, a identidade alternativa à direita.

Linearizando as identidades da alternatividade à esquerda e à direita, obtemos as identidades

$$
(x, z, y)+(z, x, y)=0(x, y, z)+(x, z, y)=0,
$$

das quais, resulta que em uma álgebra alternativa, o associador é uma função antissimétrica dos seus argumentos. Também, essas identidades podem ser escritas como

$$
(x \circ z) y-x(z y)-z(x y)=0, \quad(x y) z+(x z) y-x(y \circ z)=0 .
$$

Os exemplos mais importante de uma álgebra alternativa são as álgebras com composição.

Lembremos que em cada álgebra com composição $\mathcal{A}$, além da forma quadrática $n(x)$ (norma de $x \in \mathcal{A}$ ), estão também definidas, a forma bilinear $f(x, y)$ conectada com $n(x)$ e a forma linear traça $t(x)=f(1, x)$. Além disso, a aplicação $x \longmapsto \bar{x}=f(1, x)-x$, é uma involução na álgebra $\mathcal{A}$. Se $\mathcal{B}$ é algum subespaço da álgebra com composição $\mathcal{A}$, então, denotamos por $\mathcal{B}^{\perp}$, ao complemento ortogonal do espaço $\mathcal{B}$ com respeito à forma $f(x, y)$.

Pelo processo de duplicação de Cayley-Dickson [19] (veja Cap. II), as álgebras com composição diferentes do corpo $F$, podem ser apresentadas na forma:

$$
\begin{gathered}
K(u)=F+F v_{1}, \\
v_{1}^{2}=v_{1}+u, \quad 4 u+1 \neq 0
\end{gathered}
$$

a generalizada álgebra dos quaterniões

$$
\begin{gathered}
Q(u, \beta)=(K(u), \beta)=K(u)+v_{2} K(u), \\
v_{2} \in K(u)^{\perp}, \quad v_{2}^{2}=\beta \neq 0
\end{gathered}
$$

a álgebra de Cayley-Dickson

$$
\begin{gathered}
C(u, \beta, \gamma)=(Q(u, \beta), \gamma)=Q(u, \beta)+v_{3} Q(u, \beta), \\
v_{3} \in Q(u, \beta)^{\perp}, \quad v_{2}^{2}=\gamma \neq 0 .
\end{gathered}
$$

Denotamos por $\mathbb{H}$ e $\mathbb{O}$, respectivamente, a generalizada álgebra dos quaterniões $Q(u, \beta)$ e a 
álgebra de Cayley-Dickson $C(u, \beta, \gamma)$.

Além disso, se denotarmos as álgebras de acima por $\mathcal{A}_{v}=\mathcal{A}+\mathcal{A} v \operatorname{com} v=v_{1}, v_{2}, v_{3}$, introduzimos a involução

$$
x+y v \longmapsto \bar{x}-y v
$$

chamada $-I$, onde $x \longmapsto \bar{x}$ é a involução da álgebra com composição $\mathcal{A}$, onde $\mathcal{A}=$ $F, K(u), \mathbb{H}$.

Em uma álgebra com composição $\mathcal{A}$, as seguintes condições, são equivalentes:

(a) $n(x)=0$ para algum $x \neq 0$ de $\mathcal{A}$;

(b) existem divisores de zero.

Se uma álgebra com composição $\mathcal{A}$ satisfaz uma das condições anteriores $(a)$ ou $(b)$, então $\mathcal{A}$ é chamada split.

Exemplo 6 Consideremos a álgebra $M_{2}(F)$ de matrizes de ordem $2 \times 2$ com entradas no corpo $F$. Pode-se observar que $M_{2}(F)$ é uma álgebra com composição com respeito à forma quadrática $n(x)=\operatorname{det}(x)$. Também, em vista da presença de divisores de zero, a álgebra é split. Por [19] (veja o Teorema 6 do Cap.II), cada álgebra com composição split de dimensão 4 sobre $F$, é isomorfa a $M_{2}(F)$. Consequentemente, $\mathbb{H} \cong M_{2}(F)$. Já temos notado, que a correspondente forma quadrática sobre a álgebra $M_{2}(F)$, é a determinante da matriz. Note, também, que a forma linear traça $t(x)$ sobre a álgebra $M_{2}(F)$, é a traça usual de uma matriz. Finalmente, a involução $a \longmapsto \bar{a}=t(a)-a$, aparece na forma

$$
\overline{\left[\begin{array}{ll}
\alpha & \beta \\
\gamma & \delta
\end{array}\right]}=\left[\begin{array}{cc}
\delta & -\beta \\
-\gamma & \alpha
\end{array}\right]
$$

e é chamada a involução simplética.

Outro exemplo da generalizada álgebra dos quaterniões, é o seguinte:

Exemplo 7 Se a característica do corpo for diferente de 2, temos a clássica álgebra dos quaterniões, a qual, é uma F-álgebra de dimensão 4 com base 1,i,j, $k$ e com a seguinte tabela de multiplicação

$$
i^{2}=-1, \quad j^{2}=-1, \quad k^{2}=-1, \quad i j=-j i=k .
$$

Chamamos ao conjunto $\{1, i, j, k\}$, base clássica dos quaterniões.

\subsubsection{Bimódulos alternativos}

Sejam $\mathcal{A}$ uma álgebra alternativa sobre $F$ e $V$ um bimódulo sobre $\mathcal{A}$, isto é, $V$ é um espaço vetorial sobre $F$ equipado com as aplicações $\mathcal{A} \otimes V \longrightarrow V, a \otimes v \longmapsto a v, V \otimes \mathcal{A} \longrightarrow V$, $v \otimes a \longmapsto v a, a \in \mathcal{A}, v \in V$. Vamos a definir sobre o espaço vetorial $E=\mathcal{A} \oplus V$ uma operação binária $F$-bilinear $\cdot: E \times E \longrightarrow E$ por

$$
(a+v) \cdot(b+w)=a b+(a v+w b)
$$

onde $a, b \in \mathcal{A}, v, w \in V$. Assim, $E$ com a operação (produto) · torna-se uma álgebra, onde $\mathcal{A}$ é uma subálgebra e $V$ é um ideal tal que $V^{2}=0$. Então, dizemos que $V$ é um bimódulo alternativo sobre $\mathcal{A}$ se $E=\mathcal{A} \oplus V$ é uma álgebra alternativa com respeito a $\cdot$ Neste caso, $E=\mathcal{A} \oplus V$ é chamada a extensão cisão nula de $\mathcal{A}$ pelo bimódulo $V$. 
Portanto, um bimódulo $V$ sobre $\mathcal{A}$ é um bimódulo alternativo se, e somente se, são satisfeitas as seguintes relações

$$
\begin{aligned}
& (a, a, v)=0, \quad(a, v, b)+(v, a, b)=0 \\
& (v, b, b)=0, \quad(a, v, b)+(a, b, v)=0
\end{aligned}
$$

para todo $a, b \in \mathcal{A}, v \in V$.

Lembre que um bimódulo $V$ sobre uma álgebra de composição $\mathcal{A}$ é chamada um bimódulo de Cayley se satisfaz a relação

$$
a v=v \bar{a}
$$

onde $a \in \mathcal{A}, v \in V$, e $a \rightarrow \bar{a}$ é a involução canônica em $\mathcal{A}$.

Exemplo 8 O bimódulo de Cayley irredutível unitário não-associativo de dimensão 2 para $M_{2}(F)$ é o espaço Cay $=F . m_{1}+F . m_{2}$, onde $M_{2}(F)$ age sobre Cay por

$$
\begin{gathered}
e_{i j} \cdot m_{k}=\delta_{i k} m_{j} \\
m . a=\bar{a} . m
\end{gathered}
$$

para todo $i, j, k \in\{1,2\}$; onde $a \in M_{2}(F), m \in$ Cay e $a \mapsto \bar{a}$ é a involução simplética em $M_{2}(F)$.

Um caso concreto do exemplo anterior, é o seguinte:

Exemplo $9 \mathrm{Na}$ álgebra de matrizes de Cayley-Dickson $C(F)$, o espaço $V=F e_{12}^{(2)}+F e_{21}^{(3)}$ é um bimódulo de Cayley sobre $M_{2}(F)$, onde a ação de $M_{2}(F)$ sobre $V$ está dada no exemplo 8, e a qual, pode-se mostrar usando a tabela de multiplicação da álgebra $C(F)$.

\subsection{Formulação do problema}

No capítulo 2, temos mostrado que o Teorema de Coordenatização de Wedderburn no caso $n \geq 3$ admite uma generalização formal para as álgebras alternativas (veja o Corolário 11 desse capítulo), isto é,

Seja $\mathcal{A}$ uma álgebra alternativa com elemento identidade tal que $\mathcal{A}$ contém $M_{n}(F)(n \geq 3)$ com o mesmo elemento identidade. Então, $\mathcal{A}$ é associativa.

Observamos que nesse caso, é válido o Teorema de Coordenatização de Wedderburn, e assim, a álgebra alternativa é uma álgebra de matrizes. A partir desse resultado, foi natural a pergunta do caso $n=2$, isto é, quando $\mathbb{H} \cong M_{2}(F)$. Em geral, para a generalizada álgebra dos quaterniões $\mathbb{H}$ isto foi formulado por Nathan Jacobson em [2] na década dos anos 50. O problema é o seguinte:

Descrever as álgebras alternativas que contém uma subálgebra $\mathbb{H}$ com o mesmo elemento identidade.

Esclarecemos que, se $\mathcal{A}$ contem $\mathbb{H}$ com o mesmo elemento identidade, a conclusão do Corolário 11 não é verdade pois a existência da álgebra de Cayley-Dickson mostra que este não é o caso. 


\subsection{Solução do problema}

O enunciado e a demonstração do seguinte resultado são análogos para álgebras do Lema 11 de [16] que foi dado para superálgebras.

Proposição 15 Seja $\mathcal{A}$ uma álgebra alternativa unitária sobre um corpo $F$ que contém uma subálgebra de composição $\mathcal{C}$ com o mesmo elemento identidade. Suponha que um subespaço $V$ de $\mathcal{A}$ é $\mathcal{C}$-invariante e satisfaz (3.3). Então, as seguintes identidades são válidas para quaisquer $a, b \in \mathcal{C}, r \in \mathcal{A}, u, v \in V$;

$$
\begin{gathered}
(a b) v=b(a v), v(a b)=(v b) a, \\
a(u r)=u(\bar{a} r), \\
a(u v)=u(v a),(u v) a=(a u) v, \\
(u, v, a)=[u v, a]
\end{gathered}
$$

Demonstração: Utilizando a linearizada identidade da alternatividade à direita e por (3.3)

$$
(a b) v=a(v \circ b)-(a v) b=a((b+\bar{b}) v))-\bar{b}(a v)=(b+\bar{b})(a v)-\bar{b}(a v)=b(a v),
$$

pois $b+\bar{b}=t(b) \in F$. Por (3.3), também obtemos a segunda relação de (3.4). Além disso, pela linearizada identidade da alternatividade à esquerda

$$
a(u r)=-u(a r)+(a \circ u) r=-u(a r)+t(a)(u r)=u(\bar{a} r),
$$

o qual mostra (3.5). Substituindo $v$ pela $r$ em (3.5), obtemos a primeira equação de (3.6). De maneira semelhante, podemos obter a segunda. Finalmente,

$$
(u, v, a)=(u v) a-u(v a)=(u v) a-a(u v)=[u v, a]
$$

o qual mostra (3.7).

Isto prova o Lema.

Q.E.D.

É importante descrever a estrutura dos $\mathbb{H}$-bimódulos alternativos unitários. Tal estrutura está dada no seguinte resultado:

Proposição 16 ([16], Lema 12) Cada $\mathbb{H}$-bimódulo alternativo unitário $V$ admite a decomposiçãa $V=V_{a} \oplus V_{c}$, onde $V_{a}$ é um $\mathbb{H}$-bimódulo associativo e $V_{c}$ é um bimódulo de Cayley sob $\mathbb{H}$; além disso, o subbimódulo $V_{c}$ coincide com o subespaço $(V, \mathbb{H}, \mathbb{H})$ e cada componente irredutivel do subbimódulo $V_{c}$ é isomorfo ao $\mathbb{H}$-módulo associativo irredutivel à direita $W$ com a seguinte ação de $\mathbb{H}:$ se $a \in \mathbb{H}$ e $v \in W$, então

$$
a v=v * a, \quad v a=\bar{a} v
$$

onde $v *$ a significa a ação associativa de $\mathbb{H}$ sob $W$ e $x \mapsto \bar{x}$ é a involução canônica em $\mathbb{H}$. Em particular, se $\mathbb{H}$ é uma álgebra de divisão, então $\operatorname{dim}_{F} W=4$, e se $\mathbb{H}=M_{2}(F)$, então $\operatorname{dim}_{F} W=2$ e $W$ é isomorfo, como um $\mathbb{H}$-bimódulo, ao bimódulo de Cayley Cay.

Denotaremos por Cay $\mathbb{H}$ ao bimódulo de Cayley para a álgebra dos quaterniões de divisão $\mathbb{H}$ e por Cay ao bimódulo de Cayley de dimensão 2 para $\mathbb{H} \cong M_{2}(F)$. Por Reg $\mathbb{H}$ denotaremos ao $\mathbb{H}$-bimódulo (associativo) regular. 
Seja $\mathcal{A}$ uma álgebra alternativa, tal que, $\mathcal{A}$ contém $\mathbb{H}$ com o mesmo elemento identidade, então podemos fazer de $\mathcal{A}$ um $\mathbb{H}$-bimódulo alternativo unitário. Logo, pela Proposição 16, $\mathcal{A}$ é completamente redutível e admite a decomposição

$$
\mathcal{A}=\mathcal{A}_{a} \oplus \mathcal{A}_{c}
$$

onde $\mathcal{A}_{a}$ é um $\mathbb{H}$-bimódulo associativo unitário e $\mathcal{A}_{c}$ é um $\mathbb{H}$-bimódulo de Cayley unitário.

Seja $Z_{a}=\left\{v \in \mathcal{A}_{a} \mid[v, \mathbb{H}]=0\right\}$. Como $\mathcal{A}_{a}$ é um $\mathbb{H}$-bimódulo associativo unitário, então como em ([8], Lema 3.3), sabemos que a categoria dos $\mathbb{H}$-bimódulos unitários é equivalente à categoria dos $\mathbb{H}^{\circ} \otimes \mathbb{H}$-módulos unitários à direita [10], onde $\mathbb{H}^{\circ}$ é a álgebra antissimétrica a $\mathbb{H}$. Como $\mathbb{H}^{\circ} \otimes \mathbb{H} \cong M_{4}(F)$, isto implica que cada $\mathbb{H}$-bimódulo unitário é completamente redutível e que quaisquer dois $\mathbb{H}$-bimódulos irredutíveis unitários, são isomorfos. O $\mathbb{H}$-bimódulo Reg $=\operatorname{Reg} \mathbb{H}$ é unitário e irredutível. Portanto, temos que o bimódulo $\mathcal{A}_{a}$ é completamente redutível e é isomorfo a uma soma direta de $\mathbb{H}$-bimódulos regulares Reg, isto é, $\mathcal{A}_{a}=\sum_{i} \oplus \operatorname{Reg}_{i}$. Logo, $\mathcal{A}_{a}$ contém um conjunto de elementos $\left\{u_{i}\right\}$ (as imagens de 1 no isomorfismo com Reg), tais que $\operatorname{Reg}_{i}=u_{i} \mathbb{H}$ com $u_{i} \in Z_{a}$, e cada elemento de $\mathcal{A}_{a}$ pode ser escrito somente em uma única da forma, da forma $\sum u_{i} a_{i}, a_{i} \in \mathbb{H}$. Agora, é claro que $Z_{a} \neq 0$ e $\mathcal{A}_{a}=Z_{a} \mathbb{H}$.

Também, pela proposição 16 , o bimódulo $\mathcal{A}_{c}$ coincide com $(\mathcal{A}, \mathbb{H}, \mathbb{H})$ e é completamente redutível; isto é, $\mathcal{A}_{c}=\sum_{j} \oplus \widetilde{\mathrm{Cay}_{j}}$, onde $\widetilde{\text { Cay }}$ é igual a Cay $\mathbb{H}$ ou a Cay. Portanto,

$$
\mathcal{A}=\left(\sum \oplus \operatorname{Reg}_{i}\right) \oplus\left(\sum \oplus{\widetilde{\mathrm{Cay}_{j}}}\right)
$$

Os enunciados e as demonstrações dos Lemas 7 e 8 são análogos para bimódulos dos Lemas 3.1 e 3.2 de [8] dadas para superbimódulos.

Lema 7 Seja $\mathcal{A}=\mathcal{A}_{a} \oplus \mathcal{A}_{c}$ a decomposição de $\mathcal{A}$ de acima. Então, para quaisquer $m, n \in \mathcal{A}_{c}$, $a \in \mathbb{H}$,

$$
(m n) a=(a m) n, \quad a(m n)=m(n a)
$$

e para quaisquer $u \in \mathcal{A}_{a}, m \in \mathcal{A}_{c}, a, b \in \mathbb{H}$,

$$
\begin{aligned}
(u m) a & =(u \bar{a}) m \\
a(m u) & =m(\bar{a} u), \\
((u m) a) b & =(u m)(b a), \\
b(a(m u)) & =(a b)(m u), \\
(u m, a, b) & =(u m)[b, a], \\
(b, a, m u) & =[b, a](m u),
\end{aligned}
$$

Demonstração: Primeiro, consideremos $m, n \in \mathcal{A}_{c}, a \in \mathbb{H}$. By (3.3), (mn) $a-($ am) $n=$ $(m n) a-(m \bar{a}) n=(m, n, a)-(m, \bar{a}, n)+m(n a-\bar{a} n)=(m, n, a)+(m, a, n)=0$. De forma análoga, $a(m n)-m(n a)=0$. Isto prova $(3.8)$

Agora, sejam $u \in \mathcal{A}_{a}, m \in \mathcal{A}_{c}, a, b \in \mathbb{H}$. Então

$$
(u m) a-(u \bar{a}) m=(u, m, a)-(u, \bar{a}, m)+u(m a-\bar{a} m)=0,
$$

e de forma semelhante, $a(m u)-m(\bar{a} u)=0$, os quais provam (3.9) e (3.10). Além disso, por 


$$
(u m) a \cdot b=(u \bar{a} \cdot m) b=(u \bar{a} \cdot \bar{b}) m=(u \cdot \overline{b a}) m=(u m)(b a),
$$

o qual prova (3.11). Similarmente, por (3.10), obtemos (3.12). Finalmente, por (3.11) y (3.12) se seguem

$$
\begin{gathered}
(u m, a, b)=((u m) a) b-(u m)(a b)=(u m)(b a)-(u m)(a b)=(u m)[b, a] \quad \mathrm{e} \\
(b, a, u m)=(b a)(m u)-b(a(m u))=(b a)(m u)-(a b)(m u)=[b, a](m u)
\end{gathered}
$$

os quais provam (3.13) e (3.14).

Isto prova o Lema.

Q.E.D.

Lema 8 Os produtos $\mathcal{A}_{a} \mathcal{A}_{a}, \mathcal{A}_{a} \mathcal{A}_{c}, \mathcal{A}_{c} \mathcal{A}_{a}$ e $\mathcal{A}_{c} \mathcal{A}_{c}$ são subespaços $\mathbb{H}$-invariantes. Além disso, $\mathcal{A}_{a} \mathcal{A}_{c}+\mathcal{A}_{c} \mathcal{A}_{a} \subseteq \mathcal{A}_{c}$ e $\mathcal{A}_{c} \mathcal{A}_{c} \subseteq \mathcal{A}_{a}$.

Demonstração: Como $\mathcal{A}_{a}$ e $\mathcal{A}_{c}$ são $\mathbb{H}$-invariantes, é suficiente provar para a primeira parte do Lema, que o produto de quaisquer subespaços $\mathbb{H}$-invariantes $U$ e $W$ é de novo $\mathbb{H}$-invariante.

Temos pela linearizada identidade da alternatividade a direita

$$
(U W) \mathbb{H} \subseteq U(W \circ \mathbb{H})+(U \mathbb{H}) W \subseteq U W
$$

e de forma semelhante $\mathbb{H}(U W) \subseteq U W$.

Agora, vamos a demonstrar que $\mathcal{A}_{a} \mathcal{A}_{c}+\mathcal{A}_{c} \mathcal{A}_{a} \subseteq \mathcal{A}_{c}$. Lembremos, que pela Proposição $14, \mathcal{A}_{c}=(\mathcal{A}, \mathbb{H}, \mathbb{H})$. Escolhamos $a, b \in \mathbb{H}$ tais que $0 \neq[a, b]^{2} \in F$, e, por $(3.14)$,

$$
\mathcal{A}_{c} \mathcal{A}_{a}=[a, b]^{2}\left(\mathcal{A}_{c} \mathcal{A}_{a}\right) \subseteq[a, b]\left(\mathcal{A}_{c} \mathcal{A}_{a}\right) \subseteq\left(a, b, \mathcal{A}_{c} \mathcal{A}_{a}\right) \subseteq(\mathbb{H}, \mathbb{H}, \mathcal{A})=\mathcal{A}_{c}
$$

e de forma semelhante $\mathcal{A}_{c} \mathcal{A}_{a} \subseteq \mathcal{A}_{c}$. Finalmente, para quaisquer $m, n \in \mathcal{A}_{c}$ e $a \in \mathbb{H}$, temos por $(3.8)$ e $(3.4)$

$$
((m n) a) b=((a m) n) b=(b(a m)) n=((a b) m) n=(m n)(a b),
$$

o qual prova que $\mathcal{A}_{c} \mathcal{A}_{c} \subseteq \mathcal{A}_{a}$.

Isto prova o Lema.

Q.E.D.

Lema $9 \mathcal{A}_{a}$ é uma subálgebra associativa de $\mathcal{A}$.

Demonstração: Fixemos elementos arbitrários $u, v, w \in Z_{a}$ e $a, b, c \in \mathbb{H}$. Consideremos as identidades

$$
\begin{gathered}
{[x, y z]=[x, y] z+y[x, z]-3(x, y, z)} \\
(x y, z, t)=x(y, z, t)+(x, z, t) y-(x, y,[z, t]),
\end{gathered}
$$

e a identidade

$$
2[(x, y, z), t]=([x, y], z, t)+([y, z], x, t)+([z, x], y, t)
$$


que é válida em cada álgebra alternativa (veja [19], Lema 3,2). Então, por (3.17)

$$
([a, b], u, v)=2[(a, b, u), v]-([b, u], a, v)-([u, a], b, v)=0 .
$$

Assim, por (3.16)

$$
(u v, a, b)=u(v, a, b)+(u, a, b) v-(u, v,[a, b])=-([a, b], u, v)=0,
$$

o qual implica, $\left(Z_{a} Z_{a}, \mathbb{H}, \mathbb{H}\right)=0$. Agora, consideremos as seguintes identidades, que são válidas em cada álgebra alternativa (veja [19] e [16])

$$
\begin{gathered}
{[x, y](x, y, z)=(x, y,(x, y, z))=-(x, y, z)[x, y]} \\
((z, w, t), x, y)=((z, x, y), w, t)+(z,(w, x, y), t) \\
+(z, w,(t, x, y))-[w,(z, t,[x, y])]+([z, t], w,[x, y]) .
\end{gathered}
$$

Pela linearização de (3.18) e escolhendo $a, b \in \mathbb{H}$ tais que $[a, b]^{2}=\alpha \in F, \alpha \neq 0$, temos para quaisquer $x \in \mathcal{A}_{a}$

$$
\begin{aligned}
{[a, b](u, x, c)=} & -[u, b](a, x, c)-[a, x](u, b, c)-[u, x](a, b, c)+(a, b,(u, x, c)) \\
& +(u, b,(a, x, c))+(a, x,(u, b, c))+(u, x,(a, b, c)) \\
= & (a, b,(u, x, c)) \\
\stackrel{(3.19)}{=} & ((u, a, b), x, c)+(u,(x, a, b), c)+(u, x,(c, a, b)) \\
& -[x,(u, c,[a, b])]+([u, c], x,[a, b])=0 .
\end{aligned}
$$

Logo, $\alpha(u, x, c)=[a, b]^{2}(u, x, c)=[a, b]([a, b](u, x, c))=0$, e assim, $(u, x, c)=0$, o qual implica, $\left(Z_{a}, \mathcal{A}_{a}, \mathbb{H}\right)=0$. Em particular, $\left(Z_{a}, Z_{a}, \mathbb{H}\right)=0$. Então, por (3.15)

$$
[a, u v]=[a, u] v+u[a, v]-3(a, u, v)=0 ;
$$

e $\operatorname{assim}\left[\mathbb{H}, Z_{a} Z_{a}\right]=0$. Portanto, $Z_{a} Z_{a} \subseteq Z_{a}$.

Usando a linearização de (3.18), temos

$$
\begin{aligned}
{[a, b](u, v, w)=} & -[a, v](u, b, w)-[u, b](a, v, w)-[u, v](a, b, w)+(a, b,(u, v, w)) \\
& +(a, v,(u, b, w))+(u, b,(a, v, w))+(u, v,(a, b, w))=0
\end{aligned}
$$

Escolha $a, b \in \mathbb{H}$ tais que $[a, b]^{2}=\alpha \in F, \alpha \neq 0$. Então

$$
\alpha(u, v, w)=[a, b]^{2}(u, v, w)=[a, b]([a, b](u, v, w))=0,
$$

e assim, $(u, v, w)=0$. Portanto, $Z_{a}$ é associativa.

Consequentemente, pela linearização da identidade de Moufang do meio e usando o fato que $\mathcal{A}_{a}$ é um $\mathbb{H}$-bimódulo associativo, obtemos

$$
\begin{aligned}
(u a)(v b) & =-(b a)(v u)+(u(a v)) b+(b(a v)) u \\
& =-(b a)(v u)+(u(v a)) b+((b a) v) u \\
& =-(b a)(v u)+((u v) a) b+(b a)(v u) \\
& =(u v)(a b) .
\end{aligned}
$$

Portanto, $\mathcal{A}_{a} \mathcal{A}_{a} \subseteq \mathcal{A}_{a}$, isto é, $\mathcal{A}_{a}$ é uma subálgebra de $\mathcal{A}$. 
Lembrando que $\left(Z_{a}, \mathcal{A}_{a}, \mathbb{H}\right)=0$, então para todo $x, y \in \mathcal{A}_{a}$, obtemos

$$
\begin{aligned}
(u a, x, y) \stackrel{(3.16)}{=} & u(a, x, y)+(u, x, y) a-(u, a,[x, y]) \\
= & u(a, x, y)+(u, x, y) a .
\end{aligned}
$$

Assim, usando a última igualdade várias vezes e o fato que $Z_{a}$ é associativa, temos

$$
\begin{aligned}
(u a, v b, w c) & =u(a, v b, w c)+(u, v b, w c) a \\
& =-u(v(b, a, w c)+(v, a, w c) b)-(v(b, u, w c)+(v, u, w c) b) a \\
& =-((w(c, v, u)+(w, v, u) c) b) a=0
\end{aligned}
$$

Portanto, $\mathcal{A}_{a}$ é uma subálgebra associativa de $\mathcal{A}$.

Isto prova o Lema.

Q.E.D.

Dos Lemas 8 e 9, se segue imediatamente o seguinte resultado.

Corolário $17 \mathcal{A}=\mathcal{A}_{a} \oplus \mathcal{A}_{c}$ é uma álgebra $\mathbb{Z}_{2}$-graduada, onde $\mathcal{A}_{a}$ é a parte par e $\mathcal{A}_{c}$ é a parte impar da $\mathbb{Z}_{2}$-graduação de $\mathcal{A}$.

No que segue vamos a usar de maneira permanente o fato de que $\mathcal{A}$ é uma álgebra alternativa $\mathbb{Z}_{2}$-graduada. Assim, temos $\left(\mathcal{A}_{c}, \mathcal{A}_{a}, \mathcal{A}_{c}\right) \subseteq \mathcal{A}_{a},\left(\mathcal{A}_{c}, \mathbb{H}, \mathcal{A}_{c}\right) \subseteq \mathcal{A}_{a}$ e $\left[Z_{a}, \mathcal{A}_{c}\right] \subseteq \mathcal{A}_{c}$.

Lema $10\left(Z_{a}, \mathcal{A}, \mathcal{A}\right)=0$.

Demonstração: Fixemos elementos arbitrários $u, v, w \in Z_{a}, m, n \in \mathcal{A}_{c}$ e $a, b, c \in \mathbb{H}$. Na prova do Lema anterior temos mostrado que $\left(Z_{a}, \mathcal{A}_{a}, \mathbb{H}\right)=0$. Assim, vamos generalizar a igualdade anterior y mostrar primeiro que

$$
\left(Z_{a}, \mathcal{A}, \mathbb{H}\right)=0 .
$$

Pelo fato de que $\mathcal{A}_{c}$ é um $\mathbb{H}$-bimódulo de Cayley, temos

$$
(a, u, m)=(a u) m-a(u m)=(a u) m-(u m) \bar{a}=(a u) m-(u a) m=[a, u] m=0,
$$

o qual prova $\left(\mathbb{H}, Z_{a}, \mathcal{A}_{c}\right)=0$. Portanto,

$$
\left(Z_{a}, \mathcal{A}, \mathbb{H}\right) \subseteq\left(Z_{a}, \mathcal{A}_{a}, \mathbb{H}\right)+\left(Z_{a}, \mathcal{A}_{c}, \mathbb{H}\right)=0
$$

o qual prova (3.20).

Além disso, consideremos a identidade

$$
([x, y], y, z)=[y,(x, y, z)]
$$

que é válida em toda álgebra alternativa. Usando sua linearização, obtemos

$$
([u, m], a, b)=-([u, a], m, b)+[m,(u, a, b)]+[a,(u, m, b)]=0 .
$$

Portanto, $\left(\left[Z_{a}, \mathcal{A}_{c}\right], \mathbb{H}, \mathbb{H}\right)=0$, isto é, $\left[Z_{a}, \mathcal{A}_{c}\right] \subseteq \mathcal{A}_{a}$. Logo

$$
\left[Z_{a}, \mathcal{A}_{c}\right] \subseteq \mathcal{A}_{a} \cap \mathcal{A}_{c}=0
$$


o qual implica $\left[Z_{a}, \mathcal{A}_{c}\right]=0$.

Pela linearização de (3.18), por (3.19) e escolhendo $a, b \in \mathbb{H}$ tais que $[a, b]^{2}=\alpha \in F$, $\alpha \neq 0$ obtemos

$$
\begin{aligned}
{[a, b](u, m, n)=} & -[u, b](a, m, n)-[a, m](u, b, n)-[u, m](a, b, n)+(a, b,(u, m, n)) \\
& +(u, b,(a, m, n))+(a, m,(u, b, n))+(u, m,(a, b, n)) \\
= & (u, m,(a, b, n)) \\
= & ((u, m, a), b, n)-((u, b, n), m, a)-(u,(m, b, n), a) \\
& +[m,(u, a,[b, n])]-([u, a], m,[b, n])=0
\end{aligned}
$$

$\operatorname{logo}, \alpha(u, m, n)=[a, b]^{2}(u, m, n)=[a, b]([a, b](u, m, n))=0$ e $(u, m, n)=0$; portanto, $\left(Z_{a}, \mathcal{A}_{c}, \mathcal{A}_{c}\right)=0$. Também

$$
\begin{aligned}
{[a, b](u, n, v)=} & -[u, b](a, n, v)-[a, n](u, b, v)-[u, n](a, b, v)+(a, b,(u, n, v)) \\
& +(u, b,(a, n, v))+(a, n,(u, b, v))+(u, n,(a, b, v)) \\
= & (a, b,(u, n, v)) \\
= & ((u, n, a), b, v)-((u, b, v), n, a)-(u,(n, b, v), a) \\
& +[n,(u, a,[b, v])]-([u, a], n,[b, v])=0,
\end{aligned}
$$

assim, $\alpha(u, n, v)=[a, b]^{2}(u, n, v)=[a, b]([a, b](u, n, v))=0$ e $(u, n, v)=0$; portanto, $\left(Z_{a}, \mathcal{A}_{c}, Z_{a}\right)=0$. Em seguida, por $(3.16)$ e $(3.20)$

$$
(u a, v, m)=u(a, v, m)+(u, v, m) a-(u, a,[v, m])=0
$$

$\operatorname{assim}\left(Z_{a} \mathbb{H}, Z_{a}, \mathcal{A}_{c}\right)=0$. Portanto, $\left(\mathcal{A}_{a}, Z_{a}, \mathcal{A}_{c}\right)=0$.

Também, usando duas vezes a identidade (3.16) e o fato que $Z_{a}$ é associativa, obtemos

$$
\begin{aligned}
(u a, v b, w) & =u(a, v b, w)+(u, v b, w) a \\
& =-u(v(b, a, w)+(v, a, w) b)-(v(b, u, w)+(v, u, w) b) a=0
\end{aligned}
$$

Assim, $\left(Z_{a} \mathbb{H}, Z_{a} \mathbb{H}, Z_{a}\right)=0 ; \operatorname{logo}\left(\mathcal{A}_{a}, \mathcal{A}_{a}, Z_{a}\right)=0$. Portanto

$$
\left(Z_{a}, \mathcal{A}, \mathcal{A}\right) \subseteq\left(Z_{a}, \mathcal{A}_{a}, \mathcal{A}_{a}\right)+\left(Z_{a}, \mathcal{A}_{c}, \mathcal{A}_{a}\right)+\left(Z_{a}, \mathcal{A}_{a}, \mathcal{A}_{c}\right)+\left(Z_{a}, \mathcal{A}_{c}, \mathcal{A}_{c}\right)=0
$$

logo, $Z_{a}$ está contido no centro associativo de $\mathcal{A}$, isto é, $Z_{a} \subseteq Z(\mathcal{A})$.

Isto prova o Lema.

Q.E.D.

Corolário $18 \mathcal{A}=Z_{a} \otimes_{F} \mathbb{H}+\mathcal{A}_{c}$.

Demonstração: Como $\mathcal{A}_{a}=\sum u_{i} \mathbb{H}$, cada elemento de $\mathcal{A}_{a}$ pode ser escrito em uma única forma, da forma $\sum u_{i} a_{i}$ com $a_{i} \in \mathbb{H}$. Sabemos que $\mathcal{A}_{a}$ é associativa. Por utro lado, seja $x=\sum u_{i} a_{i} \in Z_{a}$, então $a x=x a$ para todo $a \in \mathbb{H}$. Logo, por $\left[Z_{a}, \mathbb{H}\right]=0$ temos

$$
\sum u_{i} a a_{i}=\sum u_{i} a_{i} a
$$

assim, $a a_{i}=a_{i} a$. Mas, como $\mathbb{H}$ é central, temos $a_{i}=\alpha_{1} 1, \alpha_{i} \in F$. Então, $Z_{a}=\sum F u_{i}$. Portanto, é claro que $\mathcal{A}_{a}=Z_{a} \otimes_{F} \mathbb{H}$, onde pelo Lema $9, Z_{a}$ é associativa.

Isto prova o Corolário. 
Lema $11\left[Z_{a}, Z_{a}\right] \mathcal{A}_{c}=\mathcal{A}_{c}\left[Z_{a}, Z_{a}\right]=0$.

Demonstração: Do Lema 10 tem-se $\left(Z_{a}, \mathcal{A}, \mathcal{A}\right)=0$; em particular $\left(Z_{a}, Z_{a}, \mathcal{A}\right)=0$. Assim, pela linearização de (3.18)

$$
\begin{aligned}
{\left[Z_{a}, Z_{a}\right](\mathcal{A}, \mathcal{A}, \mathcal{A}) \subseteq } & -\left[\mathcal{A}, Z_{a}\right]\left(Z_{a}, \mathcal{A}, \mathcal{A}\right)-\left[Z_{a}, \mathcal{A}\right]\left(\mathcal{A}, Z_{a}, \mathcal{A}\right)-[\mathcal{A}, \mathcal{A}]\left(Z_{a}, Z_{a}, \mathcal{A}\right) \\
& +\left(Z_{a}, Z_{a},(\mathcal{A}, \mathcal{A}, \mathcal{A})\right)+\left(\mathcal{A}, Z_{a},\left(Z_{a}, \mathcal{A}, \mathcal{A}\right)\right)+\left(Z_{a}, \mathcal{A},\left(\mathcal{A}, Z_{a}, \mathcal{A}\right)\right) \\
& +\left(\mathcal{A}, \mathcal{A},\left(Z_{a}, Z_{a}, \mathcal{A}\right)\right)=0 .
\end{aligned}
$$

Portanto, $\left[Z_{a}, Z_{a}\right](\mathcal{A}, \mathcal{A}, \mathcal{A})=0$. De forma semelhante, $(\mathcal{A}, \mathcal{A}, \mathcal{A})\left[Z_{a}, Z_{a}\right]=0$.

Seja $w \in \mathcal{A}_{c}$, por (3.4) temos

$$
[a, b] w=(a b) w-(b a) w=(a b) w-a(b w)=(a, b, w) .
$$

para todo $a, b \in \mathbb{H}$. Logo, escolhendo $a, b \in \mathbb{H}$ tais que $[a, b]^{2}=\alpha \in F, \alpha \neq 0$. Então, por $(3.18) \alpha w=[a, b]([a, b] w)=[a, b](a, b, w)=((a, b, w), a, b) \in(\mathcal{A}, \mathcal{A}, \mathcal{A})$ e $w \in(\mathcal{A}, \mathcal{A}, \mathcal{A})$. Assim, $\left[Z_{a}, Z_{a}\right] w=0$ e $w\left[Z_{a}, Z_{a}\right]=0$, os quais implicam

$$
\left[Z_{a}, Z_{a}\right] \mathcal{A}_{c}=0 \text { e } \mathcal{A}_{c}\left[Z_{a}, Z_{a}\right]=0
$$

Isso prova o Lema.

Q.E.D.

Corolário $19 S e \mathcal{A}_{c} \neq 0$ e $A n n_{l}\left(\mathcal{A}_{c}\right)=A n n_{r}\left(\mathcal{A}_{c}\right)=0$. Então, $Z_{a}$ é una subálgebra comutativa.

Observação 20 Podemos dar uma demonstração mais direta do Lema 11 usando a $\mathbb{Z}_{2}$ graduação de $\mathcal{A}$. Na demonstração do Lema 10 temos obtido $\left[Z_{a}, \mathcal{A}_{c}\right]=0$. Assim, por (3.15) e pelo Lema 10

$$
\left[Z_{a}, Z_{a}\right] \mathcal{A}_{c} \subseteq\left[Z_{a}, Z_{a} \mathcal{A}_{c}\right]-Z_{a}\left[Z_{a}, \mathcal{A}_{c}\right]+3\left(Z_{a}, Z_{a}, \mathcal{A}_{c}\right)=0
$$

$e$

$$
\mathcal{A}_{c}\left[Z_{a}, Z_{a}\right] \subseteq\left[Z_{a}, \mathcal{A}_{c} Z_{a}\right]-\left[Z_{a}, \mathcal{A}_{c}\right] Z_{a}+3\left(Z_{a}, \mathcal{A}_{c}, Z_{a}\right)=0
$$

portanto $\left[Z_{a}, Z_{a}\right] \mathcal{A}_{c}=\mathcal{A}_{c}\left[Z_{a}, Z_{a}\right]=0$. Além disso,

$$
\begin{aligned}
& {\left[Z_{a}, Z_{a}\right]\left(\mathcal{A}_{c} \mathcal{A}_{c}\right) \subseteq\left(\left[Z_{a}, Z_{a}\right] \mathcal{A}_{c}\right) \mathcal{A}_{c}+\left(\left[Z_{a}, Z_{a}\right], \mathcal{A}_{c}, \mathcal{A}_{c}\right)=\left(\mathcal{A}_{c},\left[Z_{a}, Z_{a}\right], \mathcal{A}_{c}\right)=0} \\
& \left(\mathcal{A}_{c} \mathcal{A}_{c}\right)\left[Z_{a}, Z_{a}\right] \subseteq \mathcal{A}_{c}\left(\mathcal{A}_{c}\left[Z_{a}, Z_{a}\right]\right)+\left(\mathcal{A}_{c}, \mathcal{A}_{c},\left[Z_{a}, Z_{a}\right]\right)=\left(\mathcal{A}_{c},\left[Z_{a}, Z_{a}\right], \mathcal{A}_{c}\right)=0
\end{aligned}
$$

os quais implicam

$$
\left[Z_{a}, Z_{a}\right]\left(\mathcal{A}_{c} \mathcal{A}_{c}\right)=0 \quad e\left(\mathcal{A}_{c} \mathcal{A}_{c}\right)\left[Z_{a}, Z_{a}\right]=0
$$

Se as condições do Corolário 19 são válidas, temos que $\left[Z_{a}, Z_{a}\right]=0$. Assim, $Z_{a}$ é uma álgebra comutativa

Observação 21 Se car $F=2$, então $\mathcal{A}=\mathcal{A}_{a} \oplus \mathcal{A}_{c}$ é uma superálgebra alternativa. Por ([16], Lema 8), sabemos que se $\mathcal{A}$ for prima e não trivial, isto é, $\mathcal{A}_{c} \neq 0$, então $\operatorname{Ann}\left(\mathcal{A}_{c}\right)=0$. Portanto, pelo Corolário 19, a subálgebra $Z_{a}$ de $\mathcal{A}_{a}$, é comutativa. 
Proposição 22 Se $\mathbb{H} \cong M_{2}(F)$. Então, para todo $i \neq j$ o $\mathbb{H}$-subbimódulo associativo unitário $\mathrm{Cay}_{i} \cdot \mathrm{Cay}_{j}$ de $\mathcal{A}_{a}$ tem uma base de 4 elementos. Além disso, se $i=j$, então $\mathrm{Cay}_{i}^{2}=0$.

Demonstração: Sabemos que $\mathcal{A}_{c}=\sum \oplus \mathrm{Cay}_{j}$, onde os $\mathrm{Cay}_{i}=F \cdot m_{1}^{(i)}+F \cdot m_{2}^{(i)}$ são os bimódulos de Cayley irredutíveis sobre $\mathbb{H}$. Além disso, sabemos que a álgebra alternativa $\mathcal{A}$ é $\mathbb{Z}_{2}$-graduada, $\log$ o $\mathcal{A}_{c}^{2} \subseteq \mathcal{A}_{a}$.

As relações

$$
\mathbb{H} \mathcal{A}_{c}^{2} \subseteq\left(\mathbb{H} \circ \mathcal{A}_{c}\right) \mathcal{A}_{c}+\mathcal{A}_{c}\left(\mathbb{H} \mathcal{A}_{c}\right) \subseteq \mathcal{A}_{c}^{2}
$$

$\mathrm{e}$

$$
\mathcal{A}_{c}^{2} \mathbb{H} \subseteq-\left(\mathcal{A}_{c} \mathbb{H}\right) \mathcal{A}_{c}+\mathcal{A}_{c}\left(\mathcal{A}_{c} \circ \mathbb{H}\right) \subseteq \mathcal{A}_{c}^{2}
$$

mostram que $\mathcal{A}_{c}^{2}$ é um subbimódulo de $\mathcal{A}_{a}$. Em particular, para todo $i$ e $j$ temos que $\mathrm{Cay}_{i} \mathrm{Cay}_{j}$ são subbimódulos de $\mathcal{A}_{a}$ com $\mathrm{Cay}_{i} \mathrm{Cay}_{j} \subseteq \mathcal{A}_{a}=\sum u_{k} \mathbb{H}$. Portanto, existem escalares $\alpha_{i}, \beta_{i}, \gamma_{i}, \xi_{i} \in F$ tais que $m_{1}^{(i)} m_{1}^{(j)}=\sum_{i}\left(\alpha_{i} u_{i} e_{11}+\beta_{i} u_{i} e_{12}+\gamma_{i} u_{i} e_{21}+\xi_{i} u_{i} e_{22}\right)$. Mas, por (3.6) e (3.3)

$$
e_{22}\left(m_{1}^{(i)} m_{1}^{(j)}\right)=m_{1}^{(i)}\left(m_{1}^{(j)} \cdot e_{22}\right)=m_{1}^{(i)}\left(\bar{e}_{22} \cdot m_{1}^{(j)}\right)=m_{1}^{(i)} m_{1}^{(j)}
$$

e também

$$
\left(m_{1}^{(i)} m_{1}^{(j)}\right) e_{11}=\left(e_{11} \cdot m_{1}^{(i)}\right) m_{1}^{(j)}=m_{1}^{(i)} m_{1}^{(j)} .
$$

Assim, lembrando as propriedades da subálgebra $Z_{a}$, temos

$$
\begin{aligned}
m_{1}^{(i)} m_{1}^{(j)}=e_{22}\left(m_{1}^{(i)} m_{1}^{(j)}\right) & =\sum_{i}\left(\alpha_{i} e_{22} u_{i} e_{11}+\beta_{i} e_{22} u_{i} e_{12}+\gamma_{i} e_{22} u_{i} e_{21}+\xi_{i} e_{22} u_{i} e_{22}\right) \\
& =\sum_{i}\left(\alpha_{i} u_{i} e_{22} e_{11}+\beta_{i} u_{i} e_{22} e_{12}+\gamma_{i} u_{i} e_{22} e_{21}+\xi_{i} u_{i} e_{22} e_{22}\right) \\
& =\sum_{i}\left(\gamma_{i} u_{i} e_{21}+\xi_{i} u_{i} e_{22}\right)
\end{aligned}
$$

e

$$
m_{1}^{(i)} m_{1}^{(j)}=\left(m_{1}^{(i)} m_{1}^{(j)}\right) e_{11}=\sum_{i}\left(\gamma_{i} u_{i} e_{21} e_{11}+\xi_{i} u_{i} e_{22} e_{11}\right)=\sum_{i} \gamma_{i} u_{i} e_{21}
$$

Logo, escrevemos $m_{1}^{(i)} m_{1}^{(j)}=u_{21} e_{21}$ onde $u_{21}=\sum_{i} \gamma_{i} u_{i}$. Analogamente, existem os escalares $\alpha_{i}^{\prime}, \beta_{i}^{\prime}, \gamma_{i}^{\prime}, \xi_{i}^{\prime}$ tais que $m_{1}^{(i)} m_{2}^{(j)}=\sum_{i}\left(\alpha_{i}^{\prime} u_{i} e_{11}+\beta_{i}^{\prime} u_{i} e_{12}+\gamma_{i}^{\prime} u_{i} e_{21}+\xi_{i}^{\prime} u_{i} e_{22}\right)$. Mas,

$$
e_{11}\left(m_{1}^{(i)} m_{2}^{(j)}\right)=m_{1}^{(i)}\left(m_{2}^{(j)} \cdot e_{11}\right)=m_{1}^{(i)}\left(\bar{e}_{11} \cdot m_{2}^{(j)}\right)=m_{1}^{(i)} m_{2}^{(j)}
$$

e também

$$
\left(m_{1}^{(i)} m_{2}^{(j)}\right) e_{11}=\left(e_{11} \cdot m_{1}^{(i)}\right) m_{2}^{(j)}=m_{1}^{(i)} m_{2}^{(j)}
$$

Portanto,

$$
\begin{aligned}
m_{1}^{(i)} m_{2}^{(j)}=e_{11}\left(m_{1}^{(i)} m_{2}^{(j)}\right) & =\sum_{i}\left(\alpha_{i}^{\prime} e_{11} u_{i} e_{11}+\beta_{i}^{\prime} e_{11} u_{i} e_{12}+\gamma_{i}^{\prime} e_{11} u_{i} e_{21}+\xi_{i}^{\prime} e_{11} u_{i} e_{22}\right) \\
& =\sum_{i}\left(\alpha_{i}^{\prime} u_{i} e_{11} e_{11}+\beta_{i}^{\prime} u_{i} e_{11} e_{12}+\gamma_{i}^{\prime} u_{i} e_{11} e_{21}+\xi_{i}^{\prime} u_{i} e_{11} e_{22}\right) \\
& =\sum_{i}\left(\alpha_{i}^{\prime} u_{i} e_{11}+\beta_{i}^{\prime} u_{i} e_{12}\right)
\end{aligned}
$$


e

$$
m_{1}^{(i)} m_{2}^{(j)}=\left(m_{1}^{(i)} m_{2}^{(j)}\right) e_{11}=\sum_{i}\left(\alpha_{i}^{\prime} u_{i} e_{11} e_{11}+\beta_{i}^{\prime} u_{i} e_{12} e_{11}\right)=\sum_{i} \alpha_{i}^{\prime} u_{i} e_{11}
$$

Logo, $m_{1}^{(i)} m_{2}^{(j)}=u_{11} e_{11}$ onde $u_{11}=\sum_{i} \alpha_{i}^{\prime} u_{i}$. Similarmente, existem os escalares $\alpha_{i}^{\prime \prime}, \beta_{i}^{\prime \prime}, \gamma_{i}^{\prime \prime}, \xi_{i}^{\prime \prime}$ tais que $m_{2}^{(i)} m_{1}^{(j)}=\sum_{i}\left(\alpha_{i}^{\prime \prime} u_{i} e_{11}+\beta_{i}^{\prime \prime} u_{i} e_{12}+\gamma_{i}^{\prime \prime} u_{i} e_{21}+\xi_{i}^{\prime \prime} u_{i} e_{22}\right)$. Mas,

$$
e_{22}\left(m_{2}^{(i)} m_{1}^{(j)}\right)=m_{2}^{(i)}\left(m_{1}^{(j)} \cdot e_{22}\right)=m_{2}^{(i)}\left(\bar{e}_{22} \cdot m_{1}^{(j)}\right)=m_{2}^{(i)} m_{1}^{(j)}
$$

e

$$
\left(m_{2}^{(i)} m_{1}^{(j)}\right) e_{22}=\left(e_{22} \cdot m_{2}^{(i)}\right) m_{1}^{(j)}=m_{2}^{(i)} m_{1}^{(j)}
$$

Logo,

$$
\begin{aligned}
m_{2}^{(i)} m_{1}^{(j)}=e_{22}\left(m_{1}^{(i)} m_{2}^{(j)}\right) & =\sum_{i}\left(\alpha_{i}^{\prime \prime} e_{22} u_{i} e_{11}+\beta_{i}^{\prime \prime} e_{22} u_{i} e_{12}+\gamma_{i}^{\prime \prime} e_{22} u_{i} e_{21}+\xi_{i}^{\prime \prime} e_{22} u_{i} e_{22}\right) \\
& =\sum_{i}\left(\alpha_{i}^{\prime \prime} u_{i} e_{22} e_{11}+\beta_{i}^{\prime \prime} u_{i} e_{22} e_{12}+\gamma_{i}^{\prime \prime} u_{i} e_{22} e_{21}+\xi_{i}^{\prime \prime} u_{i} e_{22} e_{22}\right) \\
& =\sum_{i}\left(\gamma_{i}^{\prime \prime} u_{i} e_{21}+\xi_{i}^{\prime \prime} u_{i} e_{22}\right)
\end{aligned}
$$

e

$$
m_{2}^{(i)} m_{1}^{(j)}=\left(m_{2}^{(i)} m_{1}^{(j)}\right) e_{22}=\sum_{i}\left(\gamma_{i}^{\prime \prime} u_{i} e_{21} e_{22}+\xi_{i}^{\prime \prime} u_{i} e_{22} e_{22}\right)=\sum_{i} \xi_{i}^{\prime \prime} u_{i} e_{22}
$$

Assim, $m_{2}^{(i)} m_{1}^{(j)}=u_{22} e_{22}$ onde $u_{22}=\sum_{i} \xi_{i}^{\prime \prime} u_{i}$. Finalmente, existem escalares $\alpha_{i}^{\prime \prime \prime}, \beta_{i}^{\prime \prime \prime}, \gamma_{i}^{\prime \prime \prime}, \xi_{i}^{\prime \prime \prime}$ tais que $m_{2}^{(i)} m_{2}^{(j)}=\sum_{i}\left(\alpha_{i}^{\prime \prime \prime} u_{i} e_{11}+\beta_{i}^{\prime \prime \prime} u_{i} e_{12}+\gamma_{i}^{\prime \prime \prime} u_{i} e_{21}+\xi_{i}^{\prime \prime \prime} u_{i} e_{22}\right)$. Mas,

$$
e_{11}\left(m_{2}^{(i)} m_{2}^{(j)}\right)=m_{2}^{(i)}\left(m_{2}^{(j)} \cdot e_{11}\right)=m_{2}^{(i)}\left(\bar{e}_{11} \cdot m_{2}^{(j)}\right)=m_{2}^{(i)} m_{2}^{(j)}
$$

e

$$
\left(m_{2}^{(i)} m_{2}^{(j)}\right) e_{22}=\left(e_{22} \cdot m_{2}^{(i)}\right) m_{2}^{(j)}=m_{2}^{(i)} m_{2}^{(j)}
$$

Então,

$$
\begin{aligned}
m_{2}^{(i)} m_{2}^{(j)}=e_{11}\left(m_{2}^{(i)} m_{2}^{(j)}\right) & =\sum_{i}\left(\alpha_{i}^{\prime \prime \prime} e_{11} u_{i} e_{11}+\beta_{i}^{\prime \prime \prime} e_{11} u_{i} e_{12}+\gamma_{i}^{\prime \prime \prime} e_{11} u_{i} e_{21}+\xi_{i}^{\prime \prime \prime} e_{11} u_{i} e_{22}\right) \\
& =\sum_{i}\left(\alpha_{i}^{\prime \prime \prime} u_{i} e_{11} e_{11}+\beta_{i}^{\prime \prime \prime} u_{i} e_{11} e_{12}+\gamma_{i}^{\prime \prime \prime} u_{i} e_{11} e_{21}+\xi_{i}^{\prime \prime \prime} u_{i} e_{11} e_{22}\right) \\
& =\sum_{i}\left(\alpha_{i}^{\prime \prime \prime} u_{i} e_{11}+\beta_{i}^{\prime \prime \prime} u_{i} e_{12}\right)
\end{aligned}
$$

e

$$
m_{2}^{(i)} m_{2}^{(j)}=\left(m_{2}^{(i)} m_{2}^{(j)}\right) e_{22}=\sum_{i}\left(\alpha_{i}^{\prime \prime \prime} u_{i} e_{11} e_{22}+\beta_{i}^{\prime \prime \prime} u_{i} e_{12} e_{22}\right)=\sum_{i} \beta_{i}^{\prime \prime \prime} u_{i} e_{12}
$$

Assim, $m_{2}^{(i)} m_{2}^{(j)}=u_{12} e_{12}$ onde $u_{12}=\sum_{i} \beta_{i}^{\prime \prime \prime} u_{i}$.

Ao considerar o $\mathbb{H}$-bimódulo $C=\left\{u_{11} e_{11}, u_{12} e_{12}, u_{21} e_{21}, u_{22} e_{22}\right\}$ finitamente gerado, temos $\mathrm{Cay}_{i} \mathrm{Cay}_{j} \subset C$. Como os elementos $u_{i j}$ pertencem a $Z_{a}$, então

$$
u_{11} e_{12}=\left(u_{11} e_{11}\right) e_{12}=\left(m_{1}^{(i)} m_{2}^{(j)}\right) e_{12}=\left(e_{12} \cdot m_{1}^{(i)}\right) m_{2}^{(j)}=m_{2}^{(i)} m_{2}^{(j)}=u_{12} e_{12},
$$


o qual, implica $u_{11}=u_{12}$. Também

$u_{11} e_{21}=e_{21}\left(u_{11} e_{11}\right)=e_{21}\left(m_{1}^{(i)} m_{2}^{(j)}\right)=m_{1}^{(i)}\left(m_{2}^{(j)} \cdot e_{21}\right)=m_{1}^{(i)}\left(\bar{e}_{21} \cdot m_{2}^{(j)}\right)=-m_{1}^{(i)} m_{1}^{(j)}=-u_{21} e_{21}$, do qual, temos $u_{11}=-u_{21}$. Seguidamente

$$
u_{11} e_{22}=\left(e_{21}\left(u_{11} e_{11}\right)\right) e_{12}=\left(e_{12} \cdot m_{1}^{(i)}\right)\left(m_{2}^{(j)} \cdot e_{21}\right)=m_{2}^{(i)}\left(\bar{e}_{21} \cdot m_{2}^{(j)}\right)=-m_{2}^{(i)} m_{1}^{(j)}=-u_{22} e_{22}
$$

e segue que $u_{11}=-u_{22}$. Portanto, temos mostrado que o $\mathbb{H}$-bimódulo $C$ é cíclico e pode ser gerado por quaisquer dos elementos $u_{r s}(r, s=1,2)$. Assim, $\operatorname{Cay}_{i} \operatorname{Cay}_{j}=u_{i j} \mathbb{H}$ e o elemento $u_{i j}$ esta bem definido para cada $i$ e $j$. Portanto, $\mathrm{Cay}_{i} \mathrm{Cay}_{j}$ tem uma base de 4 elementos.

Pelas relações obtidas acima, ao considerar $u_{i j}=e_{21}$, temos

$$
\begin{gathered}
m_{1}^{(i)} m_{1}^{(j)}=u_{i j} e_{21}, \\
m_{1}^{(i)} m_{2}^{(j)}=-u_{i j} e_{11}, \\
m_{2}^{(i)} m_{1}^{(j)}=u_{i j} e_{22}, \\
m_{2}^{(i)} m_{2}^{(j)}=-u_{i j} e_{12} .
\end{gathered}
$$

Por outro lado, se $i=j$ sabemos que $\left(m_{1}^{(i)}\right)^{2}=u_{i i} e_{21}$. Então, pela seguinte identidade

$$
\left[x^{2}, y\right]=[x, x \circ y]
$$

que é válida en toda álgebra alternativa, obtemos

$$
\left[\left(m_{1}^{(i)}\right)^{2}, e_{12}\right]=\left[m_{1}^{(i)}, m_{1}^{(i)} \circ e_{12}\right]=\left[m_{1}^{(i)}, m_{1}^{(i)} \cdot e_{12}+e_{12} \cdot m_{1}^{(i)}\right]=\left[m_{1}^{(i)}, \bar{e}_{12} \cdot m_{1}^{(i)}+e_{12} \cdot m_{1}^{(i)}\right]=0 .
$$

Imediatamente, por (3.15)

$$
0=\left[u_{i i} e_{21}, e_{12}\right]=-\left[e_{12}, u_{i i}\right] e_{21}-u_{i i}\left[e_{12}, e_{21}\right]+3\left(e_{12}, u_{i i}, e_{21}\right)=-u_{i i}\left(e_{11}-e_{22}\right) .
$$

Logo, se segue facilmente que $u_{i i}=0$. Portanto, $\mathrm{Cay}_{i}^{2}=0$.

Isto prova o Lema.

Q.E.D.

Lema 12 Para cada $i, j \neq$, temos Cay $_{i} C_{a y}=u_{i j} \mathbb{H}$ e Cay Cay $_{i}=u_{j i} \mathbb{H}$. Então,

$$
u_{j i}=-u_{i j} \text {. }
$$

Demonstração: Temos $\mathrm{Cay}_{i} \mathrm{Cay}_{j}=u_{i j} \mathbb{H}$, onde o elemento $u_{i j} \in Z_{a}$ esta bem definido e é um dos elementos $u_{11}, u_{12}, u_{21}$ e $u_{22}$ de $Z_{a}$. De forma semelhante, $\mathrm{Cay}_{j} \mathrm{Cay}_{i}=u_{j i} \mathbb{H}$ com $u_{j i} \in Z_{a}$. Assim,

$$
m_{1}^{(i)} m_{1}^{(j)}=u_{i j} e_{12}=e_{12} u_{i j} \text { e } m_{1}^{(j)} m_{1}^{(i)}=u_{j i} e_{12}=e_{12} u_{j i} .
$$

Primeiramente, vamos mostrar que $m_{1}^{(i)} \circ m_{1}^{(j)}=0$. Por $(3.6)$ e $(3,3)$

$$
e_{21}\left(m_{1}^{(i)} \circ m_{1}^{(j)}\right)=m_{1}^{(i)}\left(m_{1}^{(j)} \cdot e_{21}\right)+m_{1}^{(j)}\left(m_{1}^{(i)} \cdot e_{21}\right)=m_{1}^{(i)}\left(\bar{e}_{21} \cdot m_{1}^{(j)}\right)+m_{1}^{(i)}\left(\bar{e}_{21} \cdot m_{1}^{(j)}\right)=0 .
$$

Agora, como $m_{1}^{(i)} \circ m_{1}^{(j)} \in \mathcal{A}_{a}$, então pelo fato que $\mathcal{A}_{a}$ é um $\mathbb{H}$-bimódulo associativo, temos

$$
0=e_{12}\left(e_{21}\left(m_{1}^{(i)} \circ m_{1}^{(j)}\right)\right)=\left(e_{12} e_{21}\right)\left(m_{1}^{(i)} \circ m_{1}^{(j)}\right)=e_{11}\left(m_{1}^{(i)} \circ m_{1}^{(j)}\right) .
$$


Mas, por outro lado

$$
0=\left[e_{21}\left(m_{1}^{(i)} \circ m_{1}^{(j)}\right)\right] e_{12}=\left[e_{21}\left(e_{12} u_{i j}+e_{12} u_{j i}\right)\right] e_{12}=e_{22}\left(u_{i j} e_{12}+u_{j i} e_{12}\right)=e_{22}\left(m_{1}^{(i)} \circ m_{1}^{(j)}\right) ;
$$

assim

$$
0=e_{11}\left(m_{1}^{(i)} \circ m_{1}^{(j)}\right)+e_{22}\left(m_{1}^{(i)} \circ m_{1}^{(j)}\right)=m_{1}^{(i)} \circ m_{1}^{(j)} .
$$

Portanto,

$$
0=m_{1}^{(i)} \circ m_{1}^{(j)}=u_{i j} e_{12}+u_{j i} e_{12}=\left(u_{i j}+u_{j i}\right) e_{12},
$$

o qual, implica facilmente que $u_{j i}=-u_{i j}$.

Isto prova o Lema.

Q.E.D.

Consideremos para cada $i, j$, o seguinte subconjunto $Z=\left\{u_{i j}\right\}$ dos elementos de $Z_{a}$ obtidos na Proposição 22, tais que, $\mathrm{Cay}_{i} \mathrm{Cay}_{i}=u_{i j} \mathbb{H}$, onde $u_{i i}=0$.

Lema $13[Z, \mathcal{A}]=0$.

Demonstração: Na prova do Lema 10 temos mostrado que $\left[Z_{a}, \mathcal{A}_{c}\right]=0$. Logo, em particular

$$
\left[Z, \mathcal{A}_{c}\right]=0
$$

Agora, vamos mostrar que os elementos de $Z$ comutam com os elementos de $Z_{a}$, isto é, $\left[Z, Z_{a}\right]=0$. Sejam $m_{1}^{(i)} \in \mathrm{Cay}_{i}, m_{2}^{(j)} \in \mathrm{Cay}_{j} \operatorname{com} i, j \neq$. Como $Z_{a} \subseteq N(\mathcal{A})$, então para todo $u \in Z_{a}$, obtemos

$$
\left(m_{1}^{(i)} m_{2}^{(j)}\right) u=u_{i j} e_{11} u=u_{i j} u e_{11}
$$

E analogamente $u\left(m_{1}^{(i)} m_{2}^{(j)}\right)=u u_{i j} e_{11}$. Mas $\left[Z_{a}, \mathcal{A}_{c}\right]=0, \operatorname{logo}$

$$
u\left(m_{1}^{(i)} m_{2}^{(j)}\right)=m_{1}^{(i)} u m_{2}^{(j)}=m_{1}^{(i)} m_{2}^{(j)} u=\left(m_{1}^{(i)} m_{2}^{(j)}\right) u .
$$

Assim,

$$
u u_{i j} e_{11}=u_{i j} u e_{11}
$$

o qual, implica facilmente que $\left[u, u_{i j}\right]=0$; portanto $\left[Z_{a}, Z\right]=0$.

Assim, como sabemos que $\mathcal{A}_{a}=Z_{a} \mathbb{H}$, então por (3.15)

$$
\left[Z, \mathcal{A}_{a}\right]=\left[Z, Z_{a} \mathbb{H}\right] \subseteq\left[Z, Z_{a}\right] \mathbb{H}+Z_{a}[Z, \mathbb{H}]-3\left(Z, Z_{a}, \mathbb{H}\right)=\left[Z, Z_{a}\right] \mathbb{H}=0
$$

Consequentemente, $\left[Z, \mathcal{A}_{a}\right]=0 \mathrm{e}$

$$
[Z, \mathcal{A}]=\left[Z, \mathcal{A}_{a}\right]+\left[Z, \mathcal{A}_{c}\right]=0
$$

Isto prova o Lema.

Q.E.D.

O Lema 10 junto com o Lema 13 mostram que $Z$ está contido no centro da álgebra alternativa $\mathcal{A}$.

Sejam $i, j, k, l \neq$ e consideremos os elementos $m_{1}^{(i)}, m_{1}^{(j)}$ e $m_{2}^{(k)}$ das bases dos bimódulos de Cayley irredutíveis $\mathrm{Cay}_{i}, \mathrm{Cay}_{j} \mathrm{e} \mathrm{Cay}_{k}$ de dimensão 2. Lembremos que na demonstração 
da Proposição 22, tínhamos obtido $m_{1}^{(i)} m_{1}^{(j)}=u_{i j} e_{21}$ e $m_{1}^{(i)} m_{2}^{(k)}=-u_{i k} e_{11}$. Então, usando a igualdade $\left[Z_{a}, \mathcal{A}_{c}\right]=0$ junto com a linearizada identidade alternativa à direita, obtemos

$$
\begin{aligned}
u_{i j} m_{1}^{(k)}=u_{i j}\left(e_{21} \cdot m_{2}^{(k)}\right) & =\left(u_{i j} e_{21}\right) m_{2}^{(k)} \\
& =\left(m_{1}^{(i)} m_{1}^{(j)}\right) m_{2}^{(k)} \\
& =-\left(m_{1}^{(i)} m_{2}^{(k)}\right) m_{1}^{(j)}+m_{1}^{(i)}\left(m_{1}^{(j)} \circ m_{2}^{(k)}\right) \\
& =-\left(-u_{i k} e_{11}\right) m_{1}^{(j)}+m_{1}^{(i)}\left(-u_{j k} e_{11}+u_{k j} e_{22}\right) \\
& =u_{i k}\left(e_{11} \cdot m_{1}^{(j)}\right)+u_{j k}\left(m_{1}^{(i)} \cdot e_{11}\right)+u_{k j}\left(m_{1}^{(i)} \cdot e_{22}\right) \\
& =u_{i k} m_{1}^{(j)}+u_{j k}\left(\bar{e}_{11} \cdot m_{1}^{(i)}\right)+u_{k j}\left(\bar{e}_{22} \cdot m_{1}^{(i)}\right) \\
& =u_{i k} m_{1}^{(j)}+u_{j k}\left(e_{22} \cdot m_{1}^{(i)}\right)+u_{k j}\left(e_{11} \cdot m_{1}^{(i)}\right) \\
& =u_{i k} m_{1}^{(j)}+u_{k j} m_{1}^{(i)} .
\end{aligned}
$$

Assim

$$
u_{i j} m_{1}^{(k)}=u_{i k} m_{1}^{(j)}+u_{k j} m_{1}^{(i)}
$$

De forma semelhante, podemos obter $u_{i j} m_{2}^{(k)}=u_{i k} m_{2}^{(j)}+u_{k j} m_{2}^{(i)}$. Olhamos que para achar (3.25) temos usado $m_{1}^{(k)}=e_{21} \cdot m_{2}^{(k)}$, mas se utilizarmos a relação $m_{1}^{(k)}=e_{11} \cdot m_{1}^{(k)}$, pode-se mostrar que obtemos novamente (3.25). Logo, ao multiplicar (3.25) pelo elemento $m_{1}^{(l)}$ da base de $\mathrm{Cay}_{l}$, temos

$$
u_{i j} u_{k l} e_{21}=u_{i k} u_{j l} e_{21}+u_{k j} u_{i l} e_{21},
$$

a qual implica

$$
u_{i j} u_{k l}=u_{i k} u_{j l}+u_{k j} u_{i l} .
$$

Lema 14 Consideremos a álgebra de polinômios $F\left[x_{1}, \ldots, x_{n} ; y_{1}, \ldots, y_{n}\right]$, e sejam $\alpha_{i j}=$ $\operatorname{det}\left[\begin{array}{ll}x_{i} & y_{i} \\ x_{j} & y_{j}\end{array}\right] \in F\left[x_{1}, \ldots, x_{n} ; y_{1}, \ldots, y_{n}\right]$. Então, a álgebra alg ${ }_{F}\left\langle\alpha_{i j} \mid 1 \leq i<j \leq n\right\rangle$ é uma álgebra livre módulo as relações

$$
\begin{gathered}
\alpha_{i j}=-\alpha_{j i} \\
\alpha_{i j} \alpha_{k l}=-\alpha_{k i} \alpha_{j l}+\alpha_{k j} \alpha_{i l} .
\end{gathered}
$$

Demonstração: Vamos mostrar que os $\alpha_{i j}$ satisfazem as relações. Rapidamente,

$$
\alpha_{i j}=\operatorname{det}\left[\begin{array}{ll}
x_{i} & x_{j} \\
y_{i} & y_{j}
\end{array}\right]=x_{i} y_{j}-y_{i} x_{j}=-\left(x_{j} y_{i}-y_{j} x_{i}\right)=-\operatorname{det}\left[\begin{array}{ll}
x_{j} & x_{i} \\
y_{j} & y_{i}
\end{array}\right]=-\alpha_{j i} \text {. }
$$

$\operatorname{Sejam} \alpha_{k i}=\operatorname{det}\left[\begin{array}{ll}x_{k} & x_{i} \\ y_{k} & y_{i}\end{array}\right], \quad \alpha_{j l}=\operatorname{det}\left[\begin{array}{ll}x_{j} & x_{l} \\ y_{j} & y_{l}\end{array}\right], \quad \alpha_{k j}=\operatorname{det}\left[\begin{array}{ll}x_{k} & x_{j} \\ y_{k} & y_{j}\end{array}\right]$ e $\alpha_{i l}=\operatorname{det}\left[\begin{array}{ll}x_{i} & x_{l} \\ y_{i} & y_{l}\end{array}\right]$. Então,

$$
\begin{aligned}
-\alpha_{k i} \alpha_{j l}+\alpha_{k j} \alpha_{i l} & =-\left(x_{k} y_{i}-y_{k} x_{i}\right)\left(x_{j} y_{l}-y_{j} x_{l}\right)+\left(x_{k} y_{j}-y_{k} x_{j}\right)\left(x_{i} y_{l}-y_{i} x_{l}\right) \\
& =\left(-y_{i} x_{j}+y_{j} x_{i}\right) x_{k} y_{l}+\left(-x_{i} y_{j}+x_{j} y_{i}\right) y_{k} x_{l} \\
& =\left(x_{i} y_{j}-x_{j} y_{i}\right)\left(x_{k} y_{l}-y_{l} x_{l}\right) \\
& =\alpha_{i j} \alpha_{k l} .
\end{aligned}
$$


Agora, seja $F\left[u_{i j}\right]$ uma álgebra livre módulo as relações dadas no enunciado do Lema. Assim, existe um epimorfismo $\pi: F\left[u_{i j}\right] \longrightarrow F\left[\alpha_{i j}\right] ; u_{i j} \longmapsto \alpha_{i j}$. Provaremos que ker $\pi=0$. Seja $f\left(u_{1 i}, \ldots, u_{(n-1) n}\right) \in \operatorname{ker} \pi$, então existe $k$, tal que

$$
u_{12}^{k} f\left(u_{1 i}, \ldots, u_{(n-1) n}\right) \in F\left[u_{1 i}, \ldots, u_{2 j}\right] .
$$

Se $g\left(u_{1 i}, \ldots, u_{(n-1) n}\right)=u_{12}^{k} f\left(u_{1 i}, \ldots, u_{(n-1) n}\right)$, é claro que $g\left(u_{1 i}, \ldots, u_{(n-1) n}\right) \in \operatorname{ker} \pi$, então $g\left(\alpha_{1 i}, \ldots, \alpha_{(n-1) n}\right)=0$. Logo, todos os coeficientes do polinômio $f\left(u_{1 i}, \ldots, u_{(n-1) n}\right)$ são 0 ; portanto $f\left(u_{1 i}, \ldots, u_{(n-1) n}\right)=0$.

\section{Q.E.D.}

Lema 15 Os elementos $u_{12}, \ldots, u_{1 n}, u_{23}, \ldots, u_{2 n}$ são algebricamente independentes

Demonstração: Da relação $u_{i j} u_{k l}+u_{k i} u_{j l}+u_{k j} u_{i l}=0$, temos

$$
u_{12} u_{i j}+u_{1 i} u_{j 2}+u_{1 j} u_{2 i}=0
$$

então $u_{12} \in \operatorname{alg}_{F}\left\langle u_{1 i}, u_{2 j} \mid 1<i \leq n, 2<j \leq n\right\rangle$. Vamos mostrar que os elementos $u_{1 i}, u_{2 j}$ são algebricamente independentes. Primeiramente, observamos a igualdade dos corpos de fracções

$$
F\left(x_{1}, x_{2}, y_{1}, y_{2}\right)=F\left(x_{1}, x_{2}, y_{1}, u_{12}\right),
$$

pois $y_{2}=\frac{1}{x_{1}} u_{12}+\frac{x_{2} y_{1}}{x_{1}}$. De forma semelhante, resolvendo o sistema

$$
\begin{aligned}
& u_{1 n}=x_{1} y_{n}-y_{1} x_{n} \\
& u_{2 n}=x_{2} y_{n}-y_{2} x_{n}
\end{aligned}
$$

obtemos $x_{n}=\frac{x_{2}}{x_{1} y_{2}-x_{2} y_{1}} u_{1 n}-\frac{x_{1}}{x_{1} y_{2}-x_{2} y_{1}} u_{2 n}$ e $y_{n}=\frac{y_{2}}{x_{1} y_{2}-y_{1} y_{2}} u_{1 n}-\frac{y_{1}}{x_{1} y_{2}-y_{1} x_{2}} u_{2 n} ; \log \mathrm{O}$

$$
x_{n} y_{n} \in F\left(u_{1 n}, u_{2 n}, x_{1}, x_{2}, y_{1}, y_{2}\right)=F\left(u_{1 n}, u_{2 n}, x_{1}, x_{2}, y_{1}, u_{12}\right) .
$$

Portanto,

$$
F\left(x_{1}, \ldots, x_{2}, y_{1}, \ldots, y_{2}\right)=F\left(x_{1}, x_{2}, y_{1}, u_{12}, \ldots, u_{1 n}, u_{23}, \ldots, u_{2 n}\right) .
$$

e tr.deg $F\left(x_{1}, x_{2}, y_{1}, u_{12}, \ldots, u_{1 n}, u_{23}, \ldots, u_{2 n}\right)=2 n$ (grau de transcendência), o qual, significa que os elementos $u_{12}, \ldots, u_{1 n}, u_{23}, \ldots, u_{2 n}$ são algebricamente independentes.

Q.E.D.

No que segue vamos a supor que $\mathbb{H}$ é a álgebra dos quaterniões de divisão.

Os $\mathbb{H}$-bimódulos de Cayley formam uma categoria que é isomorfa à categoria dos $\mathbb{H}$-módulos associativos à direita via o seguinte isomorfismo (veja a demonstração da Proposição 16 dada no Lema 12 de [16]): seja $L$ um $\mathbb{H}$-módulo associativo à direita com a ação $(v, a) \mapsto v * a$. Então, o espaço vetorial $L$ com as operações de bimódulo

$$
a . v=v * a, \quad v \cdot a=\bar{a} v
$$

é um bimódulo de Cayley alternativo sobre $\mathbb{H}$ que denotaremos como Cay $L$. Por outro lado, se $M$ é um $\mathbb{H}$-bimódulo de Cayley com a ação $(m, a) \mapsto a . m$, definimos uma nova ação de $\mathbb{H}$ sobre $M$ tomando $m * a=a . m$; então

$$
m *(a b)=(a b) \cdot m \stackrel{(3.4)}{=} b \cdot(a \cdot m)=(m * a) * b,
$$


o qual, mostra que $M$ é um módulo associativo unitário à direita sobre $\mathbb{H}$.

Seja $\mathbb{H}$ a álgebra dos quaterniões de divisão com base $\{1, i, j, k\}$. Assim, ao considerar a álgebra alternativa $\mathcal{A}$ como um $\mathbb{H}$-bimódulo alternativo unitário, temos pela Proposição 16 a decomposição $\mathcal{A}=\mathcal{A}_{a} \oplus \mathcal{A}_{c}$, e além disso, o $\mathbb{H}$-bimódulo de Cayley $\mathcal{A}_{c}$ é completamente redutível e $\mathcal{A}_{c}=\sum \oplus M_{r}$, onde cada $M_{r}$ é um subbimódulo irredutível de $\mathcal{A}_{c}$.

A seguir, vamos a determinar as ações de $\mathbb{H}$ sobre os bimódulos $M_{r}$. Primeiramente, sabemos que os $M_{r}$ são $\mathbb{H}$-módulos associativos à direita irredutíveis (sobre a ação $(v, a) \mapsto$ $v * a$, e a prova da irredutibilidade, veja a demonstração da Proposição 16 dada no Lema 12 de [16]) e isomorfos a um ideal à direita minimal $I$ de $\mathbb{H}$. Mas, como $\mathbb{H}$ é uma álgebra de divisão, então $I=\mathbb{H}$ e $\operatorname{dim}_{F} I=4$. Portanto, como $\mathbb{H}$ é a álgebra dos quaterniões de divisão, então os módulos associativos à direita irredutíveis $M_{r}$ tem uma base $\left\{1_{r}, I_{r}, J_{r}, K_{r}\right\}$ sobre o qual $\mathbb{H}$ age pela seguinte tabela

\begin{tabular}{|c||c|c|c|c|}
\hline$*$ & 1 & $i$ & $j$ & $k$ \\
\hline \hline $1_{r}$ & $1_{r}$ & $I_{r}$ & $J_{r}$ & $K_{r}$ \\
\hline$I_{r}$ & $I_{r}$ & $-1_{r}$ & $K_{r}$ & $-J_{r}$ \\
\hline$J_{r}$ & $J_{r}$ & $-K_{r}$ & $-1_{r}$ & $I_{r}$ \\
\hline$K_{r}$ & $K_{r}$ & $J_{r}$ & $-I_{r}$ & $-1_{r}$ \\
\hline
\end{tabular}

Assim, se $m \in M_{r}$ e $a \in \mathbb{H}$. Das relações de acima

$$
a . m=m * a \text { e } m . a=\bar{a} \cdot m
$$

podemos obter a estrutura do $\mathbb{H}$-bimódulo de Cayley $M_{r}=$ Cay $\mathbb{H}$ a partir do $\mathbb{H}$-módulo associativo à direita $M_{r}$, isto é, temos as ações à esquerda e à direita da álgebra de divisão $\mathbb{H}$ sobre o bimódulo $M_{r}$

\begin{tabular}{|c||c|c|c|c|}
\hline$\cdot$ & $1_{r}$ & $I_{r}$ & $J_{r}$ & $K_{r}$ \\
\hline \hline 1 & $1_{r}$ & $I_{r}$ & $J_{r}$ & $K_{r}$ \\
\hline$i$ & $I_{r}$ & $-1_{r}$ & $-K_{r}$ & $J_{r}$ \\
\hline$j$ & $J_{r}$ & $K_{r}$ & $-1_{r}$ & $-I_{r}$ \\
\hline$k$ & $K_{r}$ & $-J_{r}$ & $I_{r}$ & $-1_{r}$ \\
\hline
\end{tabular}

\begin{tabular}{|c||c|c|c|c|}
\hline$\cdot$ & 1 & $i$ & $j$ & $k$ \\
\hline \hline $1_{r}$ & $1_{r}$ & $-I_{r}$ & $-J_{r}$ & $-K_{r}$ \\
\hline$I_{r}$ & $I_{r}$ & $1_{r}$ & $-K_{r}$ & $J_{r}$ \\
\hline$J_{r}$ & $J_{r}$ & $K_{r}$ & $1_{r}$ & $-I_{r}$ \\
\hline$K_{r}$ & $K_{r}$ & $-J_{r}$ & $I_{r}$ & $1_{r}$ \\
\hline
\end{tabular}

Seja $M_{s}$ outro subbimódulo irredutível da decomposição do $\mathbb{H}$-bimódulo de Cayley $\mathcal{A}_{c}$ com base $\left\{1_{s}, I_{s}, J_{s}, K_{s}\right\}$. Segue-se de (3.8) que

$$
\begin{aligned}
& \left(M_{r} M_{s}\right) \mathbb{H}=\left(\mathbb{H} M_{r}\right) M_{S} \subseteq M_{r} M_{s}, \\
& \mathbb{H}\left(M_{r} M_{s}\right)=M_{r}\left(M_{s} \mathbb{H}\right) \subseteq M_{r} M_{s} ;
\end{aligned}
$$

assim, os produtos $M_{r} M_{s}$ são subbimódulos do bimódulo associativo $\mathcal{A}_{a}$ e cada um deles tem posto menor ou igual do que $16=4 \times 4$.

Proposição 23 Para todo $r, s$ os $\mathbb{H}$-subbimódulos $M_{r} M_{s}$ têm uma base de 4 elementos. Além disso, $M_{r} M_{s}=w_{r s}^{1} \mathbb{H}+w_{r s}^{2} \mathbb{H}+w_{r s}^{3} \mathbb{H}+w_{r s}^{4} \mathbb{H}$ com $w_{r s}^{i} \in Z_{a}, i=1,2,3,4$.

Demonstração: Sabemos pelo Lema 8 que, $\mathcal{A}_{c} \mathcal{A}_{c} \subseteq \mathcal{A}_{a}$, onde $\mathcal{A}_{a}=\sum u_{i} \mathbb{H}$ e $\mathcal{A}_{c}=\sum M_{r}$. Sejam,

$$
M_{r}=F 1_{r}+F I_{r}+F J_{r}+F K_{r} \text { e } M_{s}=F 1_{s}+F I_{s}+F J_{s}+F K_{s},
$$

então existem escalares $\alpha_{t}, \beta_{t}, \gamma_{t}, \xi_{t} \in F$, tais que,

$$
1_{r} 1_{s}=\sum_{t} u_{t} a_{t}=\sum_{t} \alpha_{t} u_{t} 1+\beta_{t} u_{t} i+\gamma_{t} u_{t} j+\xi_{t} u_{t} k
$$


com $a_{t}=\alpha_{t} 1+\beta_{t} i+\gamma_{t} j+\xi_{t} k \in \mathbb{H}$. Fazendo, $w_{r s}^{1}=\sum_{t} \alpha_{t} u_{t}, w_{r s}^{2}=\sum_{t} \beta_{t} u_{t}, w_{r s}^{3}=\sum_{t} \gamma_{t} u_{t}$ e $w_{r s}^{4}=\sum_{t} \xi_{t} u_{t}$ elementos de $Z_{a}$, temos

$$
1_{r} 1_{s}=w_{r s}^{1} 1+w_{r s}^{2} i+w_{r s}^{3} j+w_{r s}^{4} k .
$$

Assim,

$$
i\left(\left(1_{r} 1_{s}\right) i\right)=-w_{r s}^{1} 1-w_{r s}^{2} i+w_{r s}^{3} j+w_{r s}^{4} k,
$$

$\operatorname{mas} i\left(\left(1_{r} 1_{s}\right) i \stackrel{(3.8)}{=}\left(i 1_{r}\right)\left(1_{s} i\right)=-I_{r} I_{s}\right.$. Então,

$$
I_{r} I_{s}=w_{r s}^{1} 1+w_{r s}^{2} i-w_{r s}^{3} j-w_{r s}^{4} k .
$$

De forma análoga, podemos obter

$$
\begin{aligned}
& J_{r} J_{s}=w_{r s}^{1} 1-w_{r s}^{2} i+w_{r s}^{3} j-w_{r s}^{4} k \\
& K_{r} K_{s}=w_{r s}^{1} 1-w_{r s}^{2} i-w_{r s}^{3} j+w_{r s}^{4} k
\end{aligned}
$$

e observamos que $1_{r} 1_{s}, I_{r} I_{s}, J_{r} J_{s}$ e $K_{r} K_{s}$ são diferentes. De forma semelhante não é difícil obter os outros elementos da base de $M_{r} M_{s}$ a partir da expressão de $1_{r} 1_{s}$, isto é, podemos obter $1_{r} I_{s}, 1_{r} J_{s}, 1_{r} K_{s}, \ldots, K_{r} K_{s}$ a partir de $1_{r} 1_{s}$ e todos são diferentes. Logo,

$$
\begin{gathered}
1_{r} 1_{s}=w_{r s}^{1} 1+w_{r s}^{2} i+w_{r s}^{3} j+w_{r s}^{4} k, \\
\vdots \\
J_{r} K_{s}=w_{r s}^{1} i+w_{r s}^{2} 1+w_{r s}^{3} k+w_{r s}^{4} j, \\
\vdots \\
K_{r} I_{s}=w_{r s}^{1} j+w_{r s}^{2} k+w_{r s}^{3} 1+w_{r s}^{4} i, \\
\vdots \\
I_{r} J_{s}=w_{r s}^{1} k+w_{r s}^{2} j+w_{r s}^{3} i+w_{r s}^{4} 1 .
\end{gathered}
$$

Portanto, em geral de (3.27) temos

$$
M_{r} M_{s}=w_{r s}^{1} \mathbb{H}+w_{r s}^{2} \mathbb{H}+w_{r s}^{3} \mathbb{H}+w_{r s}^{4} \mathbb{H} .
$$

Isto prova o Lema.

Q.E.D.

\subsection{1 Álgebras alternativas tensoriais}

Vamos descrever a categoria das álgebras alternativas sobre uma fixada álgebra alternativa $\mathcal{A}$. Esta noção foi introduzida para as álgebras de Jordan por I. Kashuba, S. Ovsienko e I. Shestakov (veja [5]) cuja fonte de inspiração foi a analogia com a álgebra associativa e o significado categórico das construções correspondentes.

Vamos estudar a noção da álgebra tensorial $\mathcal{L}\langle V\rangle$ de um bimódulo (no sentido de Eilemberg) $V$ sobre a $F$-álgebra $\mathcal{L}$. Podemos defini-la como um objeto livre em uma categoria conveniente, ou construí-la como uma álgebra fator da álgebra $F$-linear livre $\mathcal{F}=\mathcal{F}[\mathcal{L} \oplus V]$ da correspondente variedade, gerada por $\mathcal{L} \oplus V$ módulo o ideal, gerado pelas relações

$$
\left[a_{1}\right] \star\left[a_{2}\right]-\left[a_{1} \circ a_{2}\right], a_{1}, a_{2} \in \mathcal{L}
$$




$$
[v] \star[a]-[v . a],[a] \star[v]-[a . v], a \in \mathcal{L}, v \in V,
$$

onde $[x]$ significa um gerador de $\mathcal{F}$, correspondente a $x \in \mathcal{L} \oplus V$, significa o produto em $\mathcal{F}$, o o produto em $\mathcal{L}$ e $\cdot$ a ação de $\mathcal{L}$ sobre $V$. Se a álgebra $\mathcal{L}$ é associativa e $V$ é um $\mathcal{L}$-bimódulo associativo unitário, esta construção nos dá a usual álgebra tensorial $T[V]$ do bimodulo $V$. Se $\mathcal{L}$ é associativa e comutativa com $V$ é um $\mathcal{L}$-bimódulo associativo comutativo unitário, obtemos a álgebra simétrica $S[V]$. No caso das álgebras alternativas, denotaremos a álgebra alternativa tensorial de um bimódulo $V$ por $\mathcal{L}[V]$.

Vamos fazer um estudo das álgebras alternativas tensoriais, análogo como foi feito para as álgebras de Jordan tensoriais em [5].

Vamos a denotar por Alt a categoria das álgebras alternativas. Por

$$
F_{\text {Alt }}: F-\bmod \longrightarrow \text { Alt }
$$

denotamos ao funtor que associa a cada espaço $M \in \operatorname{Obj}(F-\bmod )$ a álgebra alternativa livre $F_{\text {Alt }}(M)=F[M]$ sobre $F$ gerada por $M$. O funtor $F_{\text {Alt }}$ é uma adjunta à esquerda do funtor esquecedor $E:$ Alt $\longrightarrow F-\bmod$ (que associa a cada álgebra alternativa seu respectivo espaço vetorial), isto significa que para cada $M \in \operatorname{Obj}(F-\bmod )$ e $\mathcal{A} \in \operatorname{Obj}($ Alt $)$, existe uma bijeção

$$
\alpha_{M, \mathcal{A}}: \operatorname{Hom}_{\mathrm{Alt}}\left(F_{\mathrm{Alt}}(M), \mathcal{A}\right) \longrightarrow \operatorname{Hom}_{F-\bmod }(M, E(\mathcal{A}))
$$

que é natural em $M$ e $\mathcal{A}$. Isto é consequência da propriedade universal do objeto livre $F_{\text {Alt }}(M)$ (veja o Teorema 1$)$.

Fixamos $\mathcal{L} \in$ Alt e definimos a categoria $\mathbf{A l t}_{\mathcal{L}}$ das álgebras alternativas relativas ou álgebras alternativas sobre $\mathcal{L}$. Um objeto da categoria $\mathbf{A l t}_{\mathcal{L}}$ é uma álgebra alternativa $\mathcal{A}$ dotado com um homomorfismo de álgebras alternativas $\varphi: \mathcal{L} \longrightarrow \mathcal{A}$. Sejam $\mathcal{A}_{1}, \mathcal{A}_{2} \in \operatorname{Obj}\left(\mathbf{A l t}_{\mathcal{L}}\right)$, então um morfismo $f \in \operatorname{Hom}_{\text {Alt }_{\mathcal{L}}}\left(\mathcal{A}_{1}, \mathcal{A}_{2}\right)$ é um homomorfismo de álgebras alternativas $f: \mathcal{A}_{1} \longrightarrow \mathcal{A}_{2}$, tal que, o seguinte diagrama

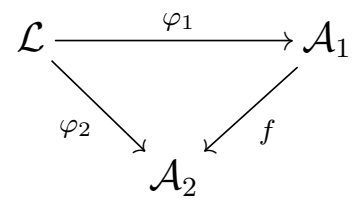

comuta, isto é, $\varphi_{2}=f \varphi_{1}$.

Usualmente na notação $\varphi: \mathcal{L} \longrightarrow \mathcal{A}$ de uma álgebra alternativa sobre $\mathcal{L}$ vamos ignorar a estrutura de homomorfismo $\varphi$ e escreveremos simplesmente por $\mathcal{A}$, e se fosse necessário denotamos ao correspondente homomorfismo por $\varphi_{\mathcal{L}}$.

Se considerarmos a generalizada álgebra dos quaterniões $\mathbb{H}$. Então, um objeto da categoria $\mathbf{A l t}_{\mathbb{H}}$ é uma álgebra alternativa $\mathcal{A}$ dotado com um homomorfismo de álgebras alternativas unitárias $\varphi: \mathbb{H} \longrightarrow \mathcal{A}$. Assim:

Problema 1 Descrever à categoria $\boldsymbol{A l t}_{\mathbb{H}}$.

Seja $\mathcal{A} \in \operatorname{Obj}\left(\mathbf{A l t}_{\mathbb{H}}\right)$, então $\mathcal{A}$ pode ser escrita como $\mathcal{A}=\mathbb{H} \oplus V$, onde $V$ é um $\mathbb{H}$-bimódulo alternativo unitário, $V=V_{a} \oplus V_{c}$. Neste caso, se $\mathcal{A}_{1}, \mathcal{A}_{2} \in \operatorname{Obj}\left(\mathbf{A l t}_{\mathbb{H}}\right)$, então $\mathcal{A}_{1}=\mathbb{H} \oplus V_{1}, \mathcal{A}_{2}=\mathbb{H} \oplus V_{2}$ e um morfismo $f \in \operatorname{Hom}_{\mathbf{A l t}_{\mathbb{H}}}\left(\mathcal{A}_{1}, \mathcal{A}_{2}\right)$ é um homomorfismo de álgebras alternativas $f: \mathcal{A}_{1} \longrightarrow \mathcal{A}_{2}$, tal que, o seguinte diagrama

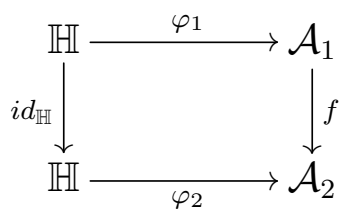


comuta, isto é, $\varphi_{2}=f \varphi_{1}$. O morfismo $f \in \operatorname{Hom}_{\mathbf{A l t}_{\mathbb{H}}}\left(\mathcal{A}_{1}, \mathcal{A}_{2}\right)$ é tal que $f_{\mathbb{H}}=i d_{\mathbb{H}}$.

Eis aqui exemplos de alguns elementos da categoria Alt $_{\mathbb{H}}$ :

Exemplo $10 S e \mathcal{A}$ é associativa, tal que, $\mathcal{A}$ contem $\mathbb{H}$ com o mesmo elmento identidade. Então, $\mathcal{A}=Z \otimes_{F} \mathbb{H}$.

Exemplo 11 Seja $\mathbb{O}$ a álgebra de Cayley-Dickson, onde $\mathbb{O}$ contem $\mathbb{H}$ e ambos têm o mesmo elemento identidade. Então, $\mathbb{O}=\mathbb{H}+v \mathbb{H}$. Aquí, v $\mathbb{H}$ é um bimódulo de Cayley sobre $\mathbb{H}$, isto é, satisfaz (3.3), onde $a \rightarrow \bar{a}$ é a involução simplética em $\mathbb{H}$. Se $\mathbb{H}$ é de divisão, então v $\mathbb{H}$ é irredutivel. Se $\mathbb{H} \cong M_{2}(F)$, então $v \mathbb{H}$ é um bimódulo de dimensão 4, isto é,

$$
v \mathbb{H}=\left\langle v e_{11}, v e_{21}\right\rangle \oplus\left\langle v e_{12}, v e_{22}\right\rangle .
$$

Exemplo 12 A subálgebra $\mathcal{A}=\mathbb{H} \oplus\left\langle v e_{11}, v e_{21}\right\rangle$ de $\mathbb{O}$, tal que, $\left\langle v e_{11}, v e_{21}\right\rangle^{2}=0$.

A seguir, fazemos uma curta descrição das somas e produtos de objetos da categoria $\mathbf{A l t}_{\mathcal{L}}$ e também descrevemos a seus objetos livres.

Consideremos duas $\mathcal{L}$-álgebras alternativas $\varphi_{1}: \mathcal{L} \longrightarrow \mathcal{A}_{1}$ a $\varphi_{2}: \mathcal{L} \longrightarrow \mathcal{A}_{2}$ e seja $F_{\text {Alt }}\left(\left[\mathcal{A}_{1}\right] \oplus\left[\mathcal{A}_{2}\right]\right)$ a álgebra alternativa livre com multiplicação $\star$. Então o produto $\mathcal{A}_{1} * \mathcal{A}_{2}$ $\left(\mathcal{A}_{1} *_{\mathcal{L}} \mathcal{A}_{2}\right)$ é o fator da álgebra $F_{\text {Alt }}\left(\left[\mathcal{A}_{1}\right] \oplus\left[\mathcal{A}_{2}\right]\right)$ pelo ideal, gerado pelas relações $\left[x_{1}\right] \star\left[y_{1}\right]-$ $\left[x_{1} \circ y_{1}\right], x_{1}, y_{1} \in \mathcal{A}_{1},\left[x_{2}\right] \star\left[y_{2}\right]-\left[x_{2} \circ y_{2}\right], x_{2}, y_{2} \in \mathcal{A}_{2},\left[\varphi_{1}(s)\right]-\left[\varphi_{2}(s)\right], s \in \mathcal{L}$, onde $\circ$ denota a multiplicação em $\mathcal{A}_{1}$ e $\mathcal{A}_{2}$ respectivamente.

Lema $16 \mathcal{A}_{1} * \mathcal{A}_{2}$ é a soma de $\mathcal{A}_{1}$ e $\mathcal{A}_{2}$ na categoria Alt $_{\mathcal{L}}$.

Para cada $\mathcal{A} \in \mathbf{A l t}_{\mathcal{L}}$, o morfismo de estrutura $\varphi_{\mathcal{A}}: \mathcal{L} \longrightarrow \mathcal{A}$ provê a $\mathcal{A}$ com a estrutura de um $\mathcal{L}$-bimódulo alternativo unitário. A seguir, damos a definição geral das álgebras alternativas tensoriais na categoria $\mathbf{A l t}_{\mathcal{L}}$.

Definição 5 Seja $V$ um bimódulo alternativo unitário sobre $\mathcal{L}$. A $\mathcal{L}$-álgebra $\mathcal{L}[V]$ junto com um homomorfismo de $\mathcal{L}$-bimódulos alternativos unitários $\imath_{V}: V \longrightarrow \mathcal{L}[V]$ é chamada uma álgebra alternativa tensorial do $\mathcal{L}$-bimódulo $V$ se para cada $\mathcal{L}$-álgebra $\mathcal{A}$ e para cada homomorfismo de $\mathcal{L}$-bimódulos alternativos unitários $f: V \longrightarrow \mathcal{A}$ existe um único homomorfismo de $\mathcal{L}$-álgebras $g: \mathcal{L}[V] \longrightarrow \mathcal{A}$, tal que, o diagrama

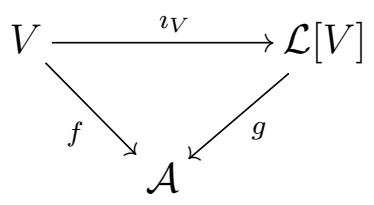

comuta, isto é, $f=g \circ \imath_{V}$.

Cada $\mathcal{A} \in \operatorname{Obj}\left(\mathbf{A l t}_{\mathcal{L}}\right)$ tem estrutura de um $\mathcal{L}$-bimódulo, que define um funtor de restrição (esquecedor) $E_{\mathcal{L}}: \mathbf{A l t}_{\mathcal{L}} \longrightarrow \mathcal{L}$-Bimod. O funtor $E_{\mathcal{L}}$ proporciona uma adjunta à esquerda $\mathcal{L}[]: \mathcal{L}-\operatorname{Bimod} \longrightarrow$ Alt,$V \longmapsto \mathcal{L}[V]$, isto significa que para cada $V \in \operatorname{Obj}(\mathcal{L}-\operatorname{Bimod})$ e $\mathcal{A} \in \operatorname{Obj}\left(\mathbf{A l t}_{\mathcal{L}}\right)$ existe uma bijeção

$$
\beta_{V, \mathcal{A}}: \operatorname{Hom}_{\mathbf{A l t}_{\mathcal{L}}}(\mathcal{L}[V], \mathcal{A}) \longrightarrow \operatorname{Hom}_{\mathcal{L}-\operatorname{Bimod}}\left(V, E_{\mathcal{L}}(\mathcal{A})\right)
$$

que é natural em $V$ e $\mathcal{A}$.

A álgebra alternativa tensorial $\mathcal{L}[V]$ do $\mathcal{L}$-bimódulo $V$ pode ser construída como um fator da álgebra alternativa libre $F_{\text {Alt }}([\mathcal{L}] \oplus[V])$ pelo ideal, gerado pelas relações $\left[a_{1}\right] \star\left[a_{2}\right]-$ $\left[a_{1} a_{2}\right], a_{1}, a_{2} \in \mathcal{L},[v] \star[a]-[v . a],[a] \star[v]-[a . v], a \in \mathcal{L}, v \in V$. A álgebra $\mathcal{L}[V]$ é canonicamente graduada por $\operatorname{deg} l=0, l \in \mathcal{L}, \operatorname{deg} v=1, v \in V$. 
Naturalmente a Definição 5 pode ser dada em qualquer variedade de álgebras.

Eis aqui exemplos de álgebras alternativas tensoriais:

Exemplo 13 Seja $V$ um bimódulo associativo unitário sobre $M_{n}(F)$. Nesse caso, sabemos que $V$ é completamente redutivel e cada componente irredutivel é isomorfo ao bimódulo regular $\operatorname{Reg}\left(M_{n}(F)\right)$. Assim, se $V=\sum_{i=1}^{m} \operatorname{Reg}\left(M_{n}(F)\right)_{i}$, então

$$
M_{n}(F)[V] \cong M_{n}\left(F\left\langle x_{1}, \ldots, x_{m}\right\rangle\right),
$$

onde $F\left\langle x_{1}, \ldots, x_{m}\right\rangle$ é a álgebra associativa livre com geradores $x_{1}, \ldots, x_{m}$.

Exemplo 14 Suponha que a característica do corpo é diferente de 2. Em [3], N. Jacobson mostro que cada bimódulo alternativo unitário sobre $\mathbb{O}$ é completamente redutivel e cada componente irredutível é isomorfo ao bimódulo regular $\operatorname{Reg}(\mathbb{O})$. Assim, se $V=\sum_{i=1}^{m} \operatorname{Reg}(\mathbb{O})_{i}$, então

$$
\mathbb{O}[V] \cong \mathbb{O} \otimes_{F} F\left[x_{1}, \ldots, x_{m}\right],
$$

onde $F\left[x_{1}, \ldots, x_{m}\right]$ é a álgebra de polinômios nas variáveis $x_{1}, \ldots, x_{m}$.

Do Corolário 8 temos

Exemplo 15 Seja $\mathcal{L}=C(\Omega)$ a álgebra matricial de Cayley-Dickson, $V=C(D)$, onde $\Omega$ é uma álgebra associativa e comutativa unitária e, $D$ é um $\Omega$-módulo associativo comutativo. Então, temos a álgebra tensorial associativo comutativo $\Omega\langle D\rangle$ do $\Omega$-módulo $D$, e

$$
\mathcal{L}[V] \cong C(\Omega\langle D\rangle)
$$

É fundamental a descrição das álgebras alternativas tensoriais na categoria $\mathbf{A l t}_{\mathbb{H}}$ pois eles irão responder o problema de Nathan Jacobson, já que cada álgebra alternativa que contenha $\mathbb{H}$ com o mesmo elemento identidade, pela propriedade universal, a álgebra alternativa será a imagem homomórfica do objeto livre.

Sabemos que para cada $\mathcal{A} \in \mathbf{A l t}_{\mathbb{H}}$, o morfismo de estrutura $\varphi_{\mathcal{A}}: \mathbb{H} \longrightarrow \mathcal{A}$ provê a $\mathcal{A}$ com a estrutura de um $\mathbb{H}$-bimódulo alternativo unitário. Portanto, pela Proposição 16, $\mathcal{A}=\mathbb{H} \oplus U$, onde $U$ é um $\mathcal{L}$-bimódulo alternativo unitário. Assim, na categoria Alt $\mathbf{t}_{\mathbb{H}}$, a definição 5 fica da seguinte maneira:

Definição 6 Seja $V$ um bimódulo alternativo unitário sobre $\mathbb{H}$. A $\mathbb{H}$-álgebra $\mathbb{H}[V]$ junto com um homomorfismo de $\mathbb{H}$-bimódulos alternativos unitários $\imath_{V}: V \longrightarrow \mathbb{H}[V]$ é chamada uma álgebra alternativa tensorial do $\mathbb{H}$-bimódulo $V$ se para cada $\mathbb{H}$-álgebra $\mathcal{A}=\mathbb{H} \oplus U$ e para cada homomorfismo de $\mathbb{H}$-bimódulos alternativos unitários $f: V \longrightarrow U$ existe um único homomorfismo de $\mathbb{H}$-álgebras $g: \mathcal{L}[V] \longrightarrow \mathcal{A}$, tal que, o diagrama

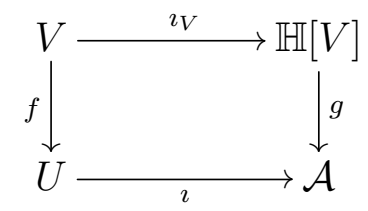

comuta, onde ı é a inclusão.

Da Definição 6 podemos dizer que o homomorfismo de álgebras $g$ estende ao homomorfismo de bimódulos $f$.

Cada $\mathcal{A} \in \operatorname{Obj}\left(\mathbf{A l t}_{\mathbb{H}}\right)$ tem a forma $\mathcal{A}=\mathbb{H} \oplus U$, onde $U$ é um $\mathbb{H}$-bimódulo alternativo unitário, que define um funtor de restrição (esquecedor) $E_{\mathbb{H}}: \mathbf{A l t}_{\mathbb{H}} \longrightarrow \mathbb{H}-$ Bimod. O funtor 
$E_{\mathbb{H}}$ proporciona uma adjunta à esquerda $\mathbb{H}[]: \mathbb{H}-\operatorname{Bimod} \longrightarrow$ Alt, $V \longmapsto \mathbb{H}[V]$, isto significa que para cada $V \in \operatorname{Obj}(\mathbb{H}-\operatorname{Bimod})$ e $\mathcal{A} \in \operatorname{Obj}\left(\mathbf{A l t}_{\mathbb{H}}\right)$ existe uma bijeção

$$
\beta_{V, \mathcal{A}}: \operatorname{Hom}_{\mathbf{A l t}_{\mathbb{H}}}(\mathbb{H}[V], \mathcal{A}) \longrightarrow \operatorname{Hom}_{\mathbb{H}-\operatorname{Bimod}}\left(V, E_{\mathbb{H}}(\mathcal{A})\right)
$$

que é natural em $V$ e $\mathcal{A}=\mathbb{H} \oplus U$, onde $E_{\mathbb{H}}(\mathcal{A})=U$.

Em nosso caso, temos

$$
\mathbb{H}[V]=\mathbb{H} \oplus \operatorname{alg}\langle V\rangle=\mathbb{H} \oplus(V+V \star V+V \star(V \star V)+(V \star V) \star V+\ldots)
$$

Agora, nosso objetivo é descrever à álgebra alternativa tensorial $\mathbb{H}[V]$ para o caso split $M_{2}(F)$. Denotemos $\mathcal{L}=M_{2}(F)$.

Seja $\mathcal{A}$ um objeto da categoria $\operatorname{Alt}_{M_{2}(F)}$, então $\mathcal{A}=M_{2}(F) \oplus V$, onde $V$ é um bimódulo alternativo unitário completamente redutível sobre $M_{2}(F)$ e $V=V_{a} \oplus V_{c} ; V_{a}$ é um $M_{2}(F)$-bimódulo associativo unitário completamente redutível e $V_{c}$ é o $M_{2}(F)$-bimódulo de Cayley completamente redutível, isto é, $V_{a}=\sum_{i=1}^{m}\left(\operatorname{Reg} M_{2}(F)\right)_{i}$ e $V_{c}=\sum_{i=1}^{n} \mathrm{Cay}_{i}$.

Portanto, para o caso split $\mathcal{L}=M_{2}(F)$, descrevemos a álgebra alternativa tensorial $\mathcal{L}[V]$, a qual, nós leva aos seguintes casos:

1) O caso puramente associativo $V=V_{a}$. Nesse caso, $\mathcal{A}=M_{2}(F) \oplus V_{a}$.

Consideremos $V=\sum_{i=1}^{m}\left(\operatorname{Reg} M_{2}(F)\right)_{i}=\sum_{i=1}^{m} u_{i} \mathcal{L}$. Consequentemente, pela demonstração do Lema 9

$$
V \star V=\left(\sum_{i=1}^{m} u_{i} \mathcal{L}\right) \star\left(\sum_{i=1}^{m} u_{i} \mathcal{L}\right)=\sum_{i_{1}, i_{2}=1}^{m} u_{i_{1}} u_{i_{2}} \mathcal{L}
$$

onde $\sum_{i_{1}, i_{2}=1}^{m} u_{i_{1}} u_{i_{2}}$ é um polinômio homogêneo de grau 2 nas variaveis $u_{1}, \ldots, u_{m}$,

$$
(V \star V) \star V=\left(\sum_{i_{1}, i_{2}=1}^{m} u_{i_{1}} u_{i_{2}} \mathcal{L}\right) \star\left(\sum_{i=1}^{m} u_{i} \mathcal{L}\right)=\sum_{i_{1}, i_{2}, i_{3}=1}^{m} u_{i_{1}} u_{i_{2}} u_{i_{3}} \mathcal{L}
$$

e claramente $V \star(V \star V)=\sum_{i_{1}, i_{2}, i_{3}=1}^{m} u_{i_{1}} u_{i_{2}} u_{i_{3}}$, donde $\sum_{i_{1}, i_{2}, i_{3}=1}^{m} u_{i_{1}} u_{i_{2}} u_{i_{3}}$ é um polinômio homogêneo de grau 3. De forma semelhante pode-se deduzir que

$$
(V \star(V \star V)) \star V=\sum_{i_{1}, i_{2}, i_{3}, i_{4}=1}^{m} u_{i_{1}} u_{i_{2}} u_{i_{3}} u_{i_{4}} \mathcal{L},
$$

neste caso $\sum_{i_{1}, i_{2}, i_{3}, i_{4}=1}^{m} u_{i_{1}} u_{i_{2}} u_{i_{3}} u_{i_{4}}$ é um polinômio homogêneo de grau 4 . Em geral, pode-se mostrar que qualquer monômio de comprimento $k$ é igual a $\sum_{i_{1}, i_{2}, \ldots, i_{k}=1}^{m} u_{i_{1}} u_{i_{2}} \ldots u_{i_{k}} \mathcal{L}$, onde $\sum_{i_{1}, i_{2}, \ldots, i_{k}=1}^{m} u_{i_{1}} u_{i_{2}} \ldots u_{i_{k}}$ é um polinômio homogêneo de grau $k$. Portanto

$$
\begin{aligned}
\mathcal{L}[V]= & \mathcal{L} \oplus(V+V \star V+V \star(V \star V)+(V \star V) \star V+((V \star V) \star V) \star V+\ldots) \\
= & \mathcal{L}+\sum_{i=1}^{m} u_{i} \mathcal{L}+\sum_{i_{1}, i_{2}=1}^{m} u_{i_{1}} u_{i_{2}} \mathcal{L}+\sum_{i_{1}, i_{2}, i_{3}=1}^{m} u_{i_{1}} u_{i_{2}} u_{i_{3}} \mathcal{L} \\
& +\sum_{i_{1}, i_{2}, i_{3}, i_{4}=1}^{m} u_{i_{1}} u_{i_{2}} u_{i_{3}} u_{i_{4}} \mathcal{L}+\ldots+\sum_{i_{1}, i_{2}, \ldots, i_{k}=1}^{m} u_{i_{1}} u_{i_{2}} \ldots u_{i_{k}} \mathcal{L}+\ldots \\
= & F\left\langle u_{1}, u_{2}, \ldots, u_{m}\right\rangle \mathcal{L} \\
\cong & M_{2}\left(F\left\langle u_{1}, u_{2}, \ldots, u_{m}\right\rangle\right) .
\end{aligned}
$$


Logo,

$$
\mathcal{L}[V] \cong M_{2}\left(F\left\langle u_{1}, u_{2}, \ldots, u_{m}\right\rangle\right),
$$

onde $F\left\langle Z_{m}\right\rangle=F\left\langle u_{1}, u_{2}, \ldots, u_{m}\right\rangle$ é a álgebra associativa livre sobre o conjunto de variáveis livres $Z_{m}=\left\{u_{1}, \ldots, u_{m}\right\}$. Isto é, a álgebra alternativa tensorial $\mathcal{L}[V]$ é isomorfa à álgebra associativa matricial $M_{2}\left(F\left\langle Z_{m}\right\rangle\right)$.

2) O caso puramente Cayley $V=V_{c}$. Nesse caso, $\mathcal{A}=M_{2}(F) \oplus V_{c}$.

Consideremos $V=\mathrm{Cay}_{1}+\cdots+\mathrm{Cay}_{n}$. De (3.25) para todo $i, j, k \neq$ temos a relação

$$
u_{i j} \mathrm{Cay}_{k} \subseteq u_{i k} \mathrm{Cay}_{j}+u_{j k} \mathrm{Cay}_{i}
$$

onde $\mathrm{Cay}_{i}=F \cdot m_{1}^{(i)}+F \cdot m_{2}^{(i)}$. Assim, por (3.28) e pela Proposição 22, junto com o Lema 13, vamos dar alguns exemplos de álgebras alternativas tensoriais

Exemplo 16 Se $n=1$, temos $V=$ Cay. Portanto, pela Proposição 22

$$
0=C a y \star C a y=V \star V=V \star(V \star V)=(V \star V) \star V=((V \star V) \star V) \star V=\ldots
$$

e dai $\mathcal{L}[V]=\mathcal{L} \oplus$ Cay é uma subálgebra de dimensão 6 da álgebra de Cayley-Dickson split.

Exemplo 17 Se $n=2$, temos $V=$ Cay $_{1} \oplus$ Cay $_{2}$. Denotemos Cay C $_{1,2} \equiv$ Cay $_{1} \oplus$ Cay $_{2}$ e pela Proposição 22, Cay $_{1,2} \star$ Cay $_{1,2}=u_{12} \mathcal{L}$ pois $u_{21}=-u_{12}$, logo temos os espaços homogêneos

$$
\begin{gathered}
V \star V=\operatorname{Cay}_{1,2} \star \operatorname{Cay}_{1,2}=u_{12} \mathcal{L}, \\
(V \star V) \star V=\left(u_{12} \mathcal{L}\right) \star C a y_{1,2}=u_{12} C a y_{1,2}, \\
V \star(V \star V)=C a y_{1,2} \star\left(u_{12} \mathcal{L}\right)=u_{12} C a y_{1,2} .
\end{gathered}
$$

Logo $(V \star V) \star V=V \star(V \star V)=u_{12}$ Cay $_{1,2}$. Também

$$
(V \star(V \star V)) \star V=\left(u_{12} \operatorname{Cay}_{1,2}\right) \star \operatorname{Cay}_{1,2}=u_{12}^{2} \mathcal{L} .
$$

Similarmente $V \star((V \star V) \star V)=(V \star V) \star(V \star V)=u_{12}^{2} \mathcal{L}$. Além disso,

$$
\begin{gathered}
V \star((V \star V) \star(V \star V))=\operatorname{Cay}_{1,2} \star\left(u_{12}^{2} \mathcal{L}\right)=u_{12}^{2} \operatorname{Cay}_{1,2}, \\
((V \star V) \star V) \star(V \star(V \star V))=u_{12} C a y_{1,2} \star u_{12} C_{a y_{1,2}}=u_{12}^{3} \mathcal{L}, \\
((V \star((V \star V) \star V)) \star V) \star(V \star V)=\left(u_{12}^{2} \mathcal{L} \star C a y_{1,2}\right) \star u_{12} \mathcal{L}=u_{12}^{2} C_{12} \star y_{12} \mathcal{L}=u_{12}^{3} \operatorname{Cay}_{1,2}, \ldots
\end{gathered}
$$

De forma semelhante podem ser obtidas as outras expressões não-associativas. Assim,

$$
\begin{aligned}
\mathcal{L}[V]= & \mathcal{L} \oplus(V+V \star V+V \star(V \star V)+(V \star V) \star V+((V \star V) \star V) \star V+\ldots) \\
= & \mathcal{L}+u_{12} \mathcal{L}+u_{12}^{2} \mathcal{L}+u_{12}^{3} \mathcal{L}+\ldots \\
& +\operatorname{Cay}_{1,2}+u_{12} \operatorname{Cay}_{1,2}+u_{12}^{2} \operatorname{Cay}_{1,2}+u_{12}^{3} \operatorname{Cay}_{1,2}+\ldots \\
= & F\left[u_{12}\right] \mathcal{L}+F\left[u_{12}\right] C_{a y}, 2 \\
\cong & F\left[u_{12}\right] \otimes_{F} \mathcal{L}+F\left[u_{12}\right] \operatorname{Cay}_{1,2} \\
\cong & M_{2}\left(F\left[u_{12}\right]\right)+F\left[u_{12}\right] \text { Cay }_{1,2}
\end{aligned}
$$

e

$$
\mathcal{L}[V] \cong M_{2}\left(F\left[u_{12}\right]\right)+F\left[u_{12}\right] \operatorname{Cay}_{1,2}
$$


$O$ conjunto dos elementos $e_{11}, e_{12}, e_{21}, e_{22}$ e os $m_{1}^{(i)}, m_{2}^{(i)}$ com $i=1,2$ é uma base da álgebra alternativa tensorial $\mathcal{L}[V]$ sobre a álgebra associativa e comutativa $F\left[u_{12}\right]$. Em efeito, suponhamos que

$$
w=a e_{11}+b e_{12}+c e_{21}+d e_{22}+\xi_{1} m_{1}^{(1)}+\xi_{2} m_{2}^{(1)}+\zeta_{1} m_{1}^{(2)}+\zeta_{2} m_{2}^{(2)}=0
$$

para alguns elementos $a, b, c, d, \xi_{i}, \zeta_{i}$ de $F\left[u_{12}\right]$ com $i=1,2$. Então,

$$
0=\left(e_{11} w\right) e_{11}=a e_{11}+\xi_{1} m_{1}^{(1)} \cdot e_{11}+\zeta_{1} m_{1}^{(2)} \cdot e_{11}=a e_{11}+\xi_{1}\left(\bar{e}_{11} \cdot m_{1}^{(1)}\right)+\zeta_{1}\left(\bar{e}_{11} \cdot m_{1}^{(2)}\right)=a e_{11}
$$

o qual implica facilmente que $a=0$. De maneira análoga, $b=c=d=0$. Logo

$$
w=\xi_{1} m_{1}^{(1)}+\xi_{2} m_{2}^{(1)}+\zeta_{1} m_{1}^{(2)}+\zeta_{2} m_{2}^{(2)}=0
$$

$e$

$$
0=m_{1}^{(1)}\left(e_{11} w\right)=\xi_{1}\left(m_{1}^{(1)}\right)^{2}+\zeta_{1}\left(m_{1}^{(1)} m_{1}^{(2)}\right)=\zeta_{1} u_{12} e_{11}
$$

Então $\zeta_{1} u_{12}=0$, isto implica que os coeficientes do polinômio $\zeta_{1}$ são zero, logo $\zeta_{1}=0$. De forma semelhante $\zeta_{2}=\xi_{i}=0$ com $i=1,2$. 20

Além disso, se considerarmos o elemento $\nu=m_{1}^{(1)}+m_{2}^{(2)}$ de Cay $y_{1,2}$, então pela Proposição

$$
\nu^{2}=\left(m_{1}^{(1)}+m_{2}^{(2)}\right)^{2}=m_{1}^{(1)} m_{2}^{(2)}+m_{2}^{(2)} m_{1}^{(1)}=-u_{12} e_{11}+u_{21} e_{22}=-u_{12}\left(e_{11}+e_{22}\right)=-u_{12} .1
$$

Assim, pelo processo de duplicação de Cayley-Dickson [19]

$$
\mathcal{L}[V] \cong M_{2}\left(F\left[u_{12}\right]\right)+\nu M_{2}\left(F\left[u_{12}\right]\right)=\left(M_{2}\left(F\left[u_{12}\right]\right), \nu\right) \cong C\left(F\left[u_{12}\right]\right)
$$

Portanto, $\mathcal{L}[V]$ é a álgebra de Cayley-Dickson sobre a álgebra associativa e comutativa $F\left[u_{12}\right]$.

Em geral, seja $V=\mathrm{Cay}_{1} \oplus \cdots \oplus \mathrm{Cay}_{n}$. De aqui em adiante vamos utilizar (3.28) para reduzir relações, como por exemplo, $u_{12} \mathrm{Cay}_{3} \subseteq u_{13} \mathrm{Cay}_{2}+u_{23} \mathrm{Cay}_{1}$. Além disso, pela Proposição $22 V \star V=\sum_{i \neq j}^{n} u_{i j} \mathcal{L}$ para $i \neq j=1, \ldots, n$, isto é, $V \star V \subseteq \mathcal{A}_{a}$. Se denotarmos $P \equiv \sum_{i \neq j}^{n} u_{i j}$, então $V \star V=P \mathcal{L}$ e pelo Lema 10 , temos os seguintes espaços homogêneos

$$
\begin{gathered}
(V \star V) \star V=P \mathcal{L} \star V=P V, \\
((V \star V) \star V) \star V=P V \star V=P^{2} \mathcal{L}, \\
(((V \star V) \star V) \star V) \star V=P^{2} \mathcal{L} \star V=P^{2} V, \\
((((V \star V) \star V) \star V) \star V) \star V=P^{2} V \star V=P^{3} \mathcal{L}, \\
((((V \star V) \star V) \star V) \star V) \star V) \star V=P^{3} \mathcal{L} \star V=P^{3} V \\
(((((V \star V) \star V) \star V) \star V) \star V) \star V) \star V=P^{3} V \star V=P^{4} \mathcal{L}
\end{gathered}
$$

e assim sucessivamente. Pelo Lema 13, não é difícil mostrar que as outras expressões não associativas com o mesmo comprimento são iguais as expressões anteriores. Além disso, utilizando o processo de acima, pode-se obter similarmente uma expressão geral para qualquer espaço homogêneo não-associativo de qualquer comprimento. Por exemplo, se o comprimento fosse par, teremos $P^{k / 2} \mathcal{L}$. Agora, se o comprimento fosse ímpar, tem-se

$$
P^{(k-1) / 2} V \text {. }
$$


Portanto, somando adequadamente os espaços homogêneos, a álgebra alternativa tensorial $\mathcal{L}[V]$ fica da seguinte forma

$$
\begin{aligned}
\mathcal{L}[V] & =\mathcal{L}+V+P \mathcal{L}+P V+P^{2} \mathcal{L}+P^{2} V+P^{3} \mathcal{L}+\ldots+P^{(k-1) / 2} V+P^{k / 2} \mathcal{L}+\ldots \\
& =\left(F \cdot 1+F . P+F . P^{2}+F . P^{3}+F . P^{4}+\ldots\right) \mathcal{L}+\left(F .1+F . P+F . P^{2}+F . P^{3}+\ldots\right) V \\
& =F\left[u_{i j}\right] \mathcal{L}+F\left[u_{i j}\right] V \\
& \cong M_{2}\left(F\left[u_{i j}\right]\right)+F\left[u_{i j}\right] V .
\end{aligned}
$$

Logo,

$$
\mathcal{L}[V] \cong M_{2}\left(F\left[u_{i j}\right]\right)+F\left[u_{i j}\right] V
$$

Mas, por (3.28)

$$
\begin{aligned}
F\left[u_{i j}\right] V= & F\left[u_{i j}\right] \mathrm{Cay}_{1,2}+F\left[u_{13}, u_{23}, \ldots, u_{1 n}, \ldots, u_{(n-1) n}\right] \mathrm{Cay}_{3} \\
& +F\left[u_{14}, u_{24}, u_{34}, \ldots, u_{1 n}, \ldots, u_{(n-1) n}\right] \mathrm{Cay}_{4}+\ldots+F\left[u_{1 n}, \ldots, u_{(n-1) n}\right] \mathrm{Cay}_{n}
\end{aligned}
$$

e observamos que os espaços

$$
F\left[u_{i j}\right], F\left[u_{13}, u_{23}, \ldots, u_{1 n}, \ldots, u_{(n-1) n}\right], \ldots, F\left[u_{1 n}, \ldots, u_{(n-1) n}\right]
$$

não são álgebras de polinômios pois as variáveis $u_{i j}$ 's satisfazem (3.26).

Seja $F\left[X_{n} \cup Y_{n}\right]$ o anel dos polinômios $F\left[x_{1}, y_{1}, \ldots, x_{n}, y_{n}\right]$ em $2 n$ variáveis. Consideremos a álgebra de Cayley-Dickson split

$$
\mathbb{O}=M_{2}(F) \oplus v M_{2}(F),
$$

e tome na extensão escalar $\mathbb{O} \otimes F\left[X_{n} \cup Y_{n}\right]$ os elementos $v_{i}=v e_{11} \otimes x_{i}+v e_{12} \otimes y_{i}, w_{i}=$ $v e_{22} \otimes y_{i}+v e_{21} \otimes x_{i}$ e seja $V_{i}=F v_{i}+F w_{i}$. É claro que $V_{i} \simeq$ Cay, além disso,

$$
V_{i} \cdot V_{j}=\alpha_{i j} M_{2}(F)
$$

onde $\alpha_{i j}=\operatorname{det}\left[\begin{array}{ll}x_{i} & y_{i} \\ x_{j} & y_{j}\end{array}\right]$. Consideremos a subálgebra $S_{n}=\operatorname{alg}_{F}\left[\alpha_{i j} \mid 1 \leq i<j \leq n\right]$ de $F\left[X_{n} \cup Y_{n}\right]$, a qual, pelo Lema 14 é uma álgebra livre módulo as relações

$$
\begin{gathered}
\alpha_{i j}=-\alpha_{j i}, \\
\alpha_{i j} \alpha_{k l}=-\alpha_{k i} \alpha_{j l}+\alpha_{k j} \alpha_{i l} .
\end{gathered}
$$

Então, $M_{2}\left(S_{n}\right)+\sum_{1}^{n} S_{n} V_{i}$ é uma subálgebra de $\mathbb{O} \otimes F\left[X_{n} \cup Y_{n}\right]$ e por (3.29) é isomorfa à álgebra alternativa tensorial $\mathcal{L}[V]$.

Além disso, a subálgebra $M_{2}\left(S_{n}\right)+\sum_{1}^{n} S_{n} V_{i}$ é livre, pois existe uma especialização

$$
\varphi: F\left[x_{1}, y_{1}, \ldots, x_{n}, y_{n}\right] \longrightarrow F
$$

definida por $x_{i} \mapsto \alpha_{i}, y_{i} \mapsto \beta_{i} \operatorname{com} v_{i j}=\operatorname{det}\left[\begin{array}{cc}\alpha_{i} & \beta_{i} \\ \alpha_{j} & \beta_{j}\end{array}\right]$ tal que, a imagem de $M_{2}\left(S_{n}\right)+\sum_{1}^{n} S_{n} V_{i}$ é a álgebra

$$
\mathcal{A}=\mathcal{L}+\mathrm{Cay}_{1}+\ldots+\mathrm{Cay}_{n}
$$

onde $\mathrm{Cay}_{i} \mathrm{Cay}_{j} \cong \mathcal{L}$. 
Finalmente chegamos ao caso onde $V_{a} \neq 0$ e $V_{c} \neq 0$.

3) O caso geral, $V=V_{a} \oplus V_{c}$. Nesse caso, $\mathcal{A}=M_{2}(F) \oplus\left(V_{a} \oplus V_{c}\right)$.

Vamos continuar usando alguns resultados mostrados nos Lemas 10 e 13, isto é, as igualdades

$$
\left(Z_{a}, \mathcal{A}, \mathcal{A}\right)=0 \quad \text { e }\left[Z_{a}, \mathcal{A}_{c}\right]=[Z, \mathcal{A}]=0
$$

No que segue vamos considerar alguns exemplos particulares para ver o comportamento da álgebra alternativa tensorial.

Exemplo 18 Vamos fazer variar $m$ e fixar $n=1$, isto é, seja qualquer $m \in \mathbb{N}$ e $n=1$, então temos $V=\sum_{i=1}^{m} u_{i} \mathcal{L}+$ Cay. Lembremos Cay ${ }^{2}=0$. Consequentemente

$$
V \star V=\left(\sum_{i=1}^{m} u_{i} \mathcal{L}+\text { Cay }\right) \star\left(\sum_{i=1}^{m} u_{i} \mathcal{L}+\text { Cay }\right)=\sum_{i_{1}, i_{2}=1}^{m} u_{i_{1}} u_{i_{2}} \mathcal{L}+\sum_{i=1}^{m} u_{i} \text { Cay }
$$

onde $\sum_{i_{1}, i_{2}=1}^{m} u_{i_{1}} u_{i_{2}}$ é um polinômio homogêneo de grau 2 nas variaveis $u_{1}, \ldots, u_{m}$,

$$
\begin{aligned}
(V \star V) \star V & =\left(\sum_{i_{1}, i_{2}=1}^{m} u_{i_{1}} u_{i_{2}} \mathcal{L}+\sum_{i=1}^{m} u_{i} \text { Cay }\right) \star\left(\sum_{i=1}^{m} u_{i} \mathcal{L}+\text { Cay }\right) \\
& =\sum_{i_{1}, i_{2}, i_{3}=1}^{m} u_{i_{1}} u_{i_{2}} u_{i_{3}} \mathcal{L}+\sum_{i_{1}, i_{2}=1}^{m} u_{i_{1}} u_{i_{2}} \text { Cay }
\end{aligned}
$$

donde $\sum_{i_{1}, i_{2}, i_{3}=1}^{m} u_{i_{1}} u_{i_{2}} u_{i_{3}}$ é um polinômio homogêneo de grau 3. Então, analogamente pode-se deduzir que

$$
((V \star V) \star V) \star V=\sum_{i_{1}, i_{2}, i_{3}, i_{4}=1}^{m} u_{i_{1}} u_{i_{2}} u_{i_{3}} u_{i_{4}} \mathcal{L}+\sum_{i_{1}, i_{2}, i_{3}=1}^{m} u_{i_{1}} u_{i_{2}} u_{i_{3}} \text { Cay }
$$

neste caso $\sum_{i_{1}, i_{2}, i_{3}, i_{4}=1}^{m} u_{i_{1}} u_{i_{2}} u_{i_{3}} u_{i_{4}}$ é um polinômio homogêneo de grau 4 . Assim, de maneira geral, para qualquer monômio não-associativo de comprimento $k$, temos

$$
\sum_{i_{1}, i_{2}, \ldots, i_{k}=1}^{m} u_{i_{1}} u_{i_{2}} \ldots u_{i_{k}} \mathcal{L}+\sum_{i_{1}, i_{2}, \ldots, i_{(k-1)}=1}^{m} u_{i_{1}} u_{i_{2}} \ldots u_{i_{(k-1)}} \text { Cay }
$$

onde $\sum_{i_{1}, i_{2}, \ldots, i_{k}=1}^{m} u_{i_{1}} u_{i_{2}} \ldots u_{i_{k}}$ é um polinômio homogêneo de grau $k$. Portanto,

$$
\begin{aligned}
& \mathcal{L}[V]= \mathcal{L} \\
&+\sum_{i=1}^{m} u_{i} \mathcal{L}+C a y+\sum_{i_{1}, i_{2}=1}^{m} u_{i_{1}} u_{i_{2}} \mathcal{L}+\sum_{i=1}^{m} u_{i} C a y+\sum_{i_{1}, i_{2}, i_{3}=1}^{m} u_{i_{1}} u_{i_{2}} u_{i_{3}} \mathcal{L}+\sum_{i_{1}, i_{2}=1}^{m} u_{i_{1}} u_{i_{2}} \text { Cay } \\
&+\sum_{i_{1}, i_{2}, i_{3}, i_{4}=1}^{m} u_{i_{1}} u_{i_{2}} u_{i_{3}} u_{i_{4}} \mathcal{L}+\sum_{i_{1}, i_{2}, i_{3}=1}^{m} u_{i_{1}} u_{i_{2}} u_{i_{3}} C a y \\
& \vdots \\
&+\sum_{i_{1}, i_{2}, \ldots, i_{k}=1}^{m} u_{i_{1}} u_{i_{2}} \ldots u_{i_{k}} \mathcal{L}+\sum_{i_{1}, i_{2}, \ldots, i_{(k-1)}=1}^{m} u_{i_{1}} u_{i_{2}} \ldots u_{i_{(k-1)}} \text { Cay }+\ldots
\end{aligned}
$$


Logo,

$$
\begin{aligned}
\mathcal{L}[V]= & \left(F \cdot 1+\sum_{i=1}^{m} F u_{i}+\sum_{i_{1}, i_{2}=1}^{m} F u_{i_{1}} u_{i_{2}}+\sum_{i_{1}, i_{2}, i_{3}=1}^{m} F u_{i_{1}} u_{i_{2}} u_{i_{3}}\right. \\
& \left.+\sum_{i_{1}, i_{2}, i_{3}, i_{4}=1}^{m} F u_{i_{1}} u_{i_{2}} u_{i_{3}} u_{i_{4}}+\ldots+\sum_{i_{1}, i_{2}, \ldots, i_{k}=1}^{m} F u_{i_{1}} u_{i_{2}} \ldots u_{i_{k}}+\ldots\right) \mathcal{L} \\
& +\left(F \cdot 1+\sum_{i=1}^{m} F u_{i}+\sum_{i_{1}, i_{2}=1}^{m} F u_{i_{1}} u_{i_{2}}+\sum_{i_{1}, i_{2}, i_{3}=1}^{m} F u_{i_{1}} u_{i_{2}} u_{i_{3}}\right. \\
& \left.+\ldots+\sum_{i_{1}, i_{2}, \ldots, i_{(k-1)}=1}^{m} F u_{i_{1}} u_{i_{2}} \ldots u_{i_{(k-1)}}+\ldots\right) \text { Cay },
\end{aligned}
$$

isto é,

$$
\mathcal{L}[V]=F\left\langle Z_{m}\right\rangle \mathcal{L}+F\left\langle Z_{m}\right\rangle C a y \cong M_{2}\left(F\left\langle Z_{m}\right\rangle\right)+F\left\langle Z_{m}\right\rangle \text { Cay }
$$

onde $F\left\langle Z_{m}\right\rangle$ Cay $=I \triangleleft \mathcal{L}[V]$ com $I^{2}=0$ e a álgebra quociente $\mathcal{L}[V] / I$ é a álgebra associativa matricial $\mathcal{L}=M_{2}(F)$. E como no exemplo 17 , pode-se mostrar que o conjunto dos elementos $e_{11}, e_{12}, e_{21}, e_{22}, m_{1}, m_{2}$ é uma base da álgebra alternativa tensoral $\mathcal{L}[V]$ sobre a álgebra associativa livre $F\left\langle Z_{m}\right\rangle$.

Exemplo 19 Agora vamos fixar $m=1$ e vamos fazer variar $n$. Então, neste caso $V=$ $u_{1} \mathcal{L}+\sum_{i=1}^{n}$ Cay $_{i}$. Aqui, como antes, vamos continuar usando (3.28) para reduzir relações. Além disso, denotamos $W \equiv V_{c}=\sum_{i=1}^{n}$ Cay , então pela Proposição $22 W^{2}=\sum_{i \neq j}^{n} u_{i j} \mathcal{L}$ para $i \neq j=1, \ldots, n$, isto é, $W^{2} \subseteq \mathcal{A}_{a}$. Se denotarmos novamente $P \equiv \sum_{i \neq j}^{n} u_{i j}$, temos os seguintes espaços homogêneos

$$
\begin{aligned}
& V \star V=\left(u_{1} \mathcal{L}+W\right) \star\left(u_{1} \mathcal{L}+W\right)=u_{1}^{2} \mathcal{L}+u_{1} W+P \mathcal{L}, \\
& (V \star V) \star V=u_{1}^{3} \mathcal{L}+u_{1}^{2} W+u_{1}^{2} W+u_{1} P \mathcal{L}+u_{1} P \mathcal{L}+P W=u_{1}^{3} \mathcal{L}+u_{1}^{2} W+u_{1} P \mathcal{L}+P W, \\
& (V \star(V \star V)) \star V=u_{1}^{4} \mathcal{L}+u_{1}^{3} W+u_{1}^{2} P \mathcal{L}+u_{1} P W+u_{1}^{3} W+u_{1}^{2} P \mathcal{L}+u_{1} P W+P^{2} \mathcal{L} \\
& =u_{1}^{4} \mathcal{L}+u_{1}^{3} W+u_{1}^{2} P \mathcal{L}+u_{1} P W+P^{2} \mathcal{L}, \\
& V \star((V \star V) \star(V \star V))=u_{1}^{5} \mathcal{L}+u_{1}^{4} W+u_{1}^{3} P \mathcal{L}+u_{1}^{2} P W+u_{1} P^{2} \mathcal{L} \\
& +u_{1}^{4} W+u_{1}^{3} P \mathcal{L}+u_{1}^{2} P W+u_{1} P^{2} \mathcal{L}+P^{2} W \\
& =u_{1}^{5} \mathcal{L}+u_{1}^{4} W+u_{1}^{3} P \mathcal{L}+u_{1}^{2} P W+u_{1} P^{2} \mathcal{L}+P^{2} W, \\
& ((V \star V) \star V) \star(V \star(V \star V))=u_{1}^{6} \mathcal{L}+u_{1}^{5} W+u_{1}^{4} P \mathcal{L}+u_{1}^{3} P W+u_{1}^{2} P^{2} \mathcal{L}+u_{1} P^{2} W \\
& +u_{1}^{5} W+u_{1}^{4} P \mathcal{L}+u_{1}^{3} P W+u_{1}^{2} P^{2} \mathcal{L}+u_{1} P^{2} W+P^{3} \mathcal{L} \\
& =u_{1}^{6} \mathcal{L}+u_{1}^{5} W+u_{1}^{4} P \mathcal{L}+u_{1}^{3} P W+u_{1}^{2} P^{2} \mathcal{L}+u_{1} P^{2} W+P^{3} \mathcal{L}, \\
& ((V \star((V \quad \star \quad V) \star V)) \star V) \star(V \star V) \\
& =u_{1}^{7} \mathcal{L}+u_{1}^{6} W+u_{1}^{5} P \mathcal{L}+u_{1}^{4} P W+u_{1}^{3} P^{2} \mathcal{L}+u_{1}^{2} P^{2} W+u_{1} P^{3} \mathcal{L} \\
& +u_{1}^{6} W+u_{1}^{5} P \mathcal{L}+u_{1}^{4} P W+u_{1}^{3} P^{2} \mathcal{L}+u_{1}^{2} P^{2} W+u_{1} P^{3} \mathcal{L}+P^{3} W \\
& =u_{1}^{7} \mathcal{L}+u_{1}^{6} W+u_{1}^{5} P \mathcal{L}+u_{1}^{4} P W+u_{1}^{3} P^{2} \mathcal{L}+u_{1}^{2} P^{2} W+u_{1} P^{3} \mathcal{L}+P^{3} W
\end{aligned}
$$


e assim sucessivamente. Utilizando o processo de acima, podemos obter uma expressão geral para qualquer espaço homogêneo não-associativo de comprimento $k$, isto é, se $k$ for par, temos

$$
\sum_{s=0}^{(k-2) / 2}\left[u_{1}^{k-2 s} P^{s} \mathcal{L}+u_{1}^{k-(2 s+1)} P^{s} W\right]+P^{k / 2} \mathcal{L} .
$$

Agora, se $k$ for impar, tem-se

$$
\sum_{s=0}^{(k-1) / 2}\left[u_{1}^{k-2 s} P^{s} \mathcal{L}+u_{1}^{k-(2 s+1)} P^{s} W\right] .
$$

Portanto, somando adequadamente os espaços homogêneos, a álgebra tensorial $\mathcal{L}[V]$ fica da seguinte forma

$$
\begin{aligned}
\mathcal{L}[V]= & \left(F \cdot 1+F u+F u^{2}+F u^{3}+F u^{4}+F u^{5}+F u^{6}+F u^{7} \ldots\right) \mathcal{L} \\
& +\left(F \cdot 1+F u+F u^{2}+F u^{3}+F u^{4}+F u^{5}+F u^{6} \ldots\right) W \\
& +\left(F \cdot 1+F u+F u^{2}+F u^{3}+F u^{4}+F u^{5} \ldots\right) P \mathcal{L} \\
& +\left(F \cdot 1+F u+F u^{2}+F u^{3}+F u^{4} \ldots\right) P W \\
& +\left(F \cdot 1+F u+F u^{2}+F u^{3} \ldots\right) P^{2} \mathcal{L} \\
& +\left(F \cdot 1+F u+F u^{2} \ldots\right) P^{2} W \\
& +(F \cdot 1+F u+\ldots) P^{3} \mathcal{L} \\
& +(F \cdot 1+\ldots) P^{3} W+\ldots
\end{aligned}
$$

$\log O$

$$
\begin{aligned}
\mathcal{L}[V]= & F\left\langle u_{1}\right\rangle \mathcal{L}+F\left\langle u_{1}\right\rangle W \\
& +F\left\langle u_{1}\right\rangle P \mathcal{L}+F\left\langle u_{1}\right\rangle P W \\
& +F\left\langle u_{1}\right\rangle P^{2} \mathcal{L}+F\left\langle u_{1}\right\rangle P^{2} W \\
& +F\left\langle u_{1}\right\rangle P^{3} \mathcal{L}+F\left\langle u_{1}\right\rangle P^{3} W+\ldots \\
= & F\left\langle u_{1}\right\rangle\left(F \cdot 1+F \cdot P+F \cdot P^{2}+F \cdot P^{3}+\ldots\right) \mathcal{L} \\
\quad & \quad+F\left\langle u_{1}\right\rangle\left(F \cdot 1+F \cdot P+F \cdot P^{2}+F \cdot P^{3}+\ldots\right) W \\
= & F\left\langle u_{1}\right\rangle F\left[u_{i j}\right] \mathcal{L}+F\left\langle u_{1}\right\rangle F\left[u_{i j}\right] W .
\end{aligned}
$$

Mas, por (3.28)

$$
\begin{gathered}
F\left[u_{i j}\right] W=F\left[u_{i j}\right] \operatorname{Cay}_{1,2}+F\left[u_{13}, u_{23}, \ldots, u_{1 n}, \ldots, u_{(n-1) n}\right] C a y_{3}+ \\
F\left[u_{14}, u_{24}, u_{34}, \ldots, u_{1 n}, \ldots, u_{(n-1) n}\right] C a y_{4}+\ldots+F\left[u_{1 n}, \ldots, u_{(n-1) n}\right] C a y_{n}
\end{gathered}
$$

onde novamente como no caso puramente Cayley, os espaços $F\left[u_{i j}\right]$ para $i \neq j=1, \ldots, n$, $F\left[u_{13}, u_{23}, \ldots, u_{1 n}, \ldots, u_{(n-1) n}\right], F\left[u_{14}, u_{24}, u_{34}, \ldots, u_{1 n}, \ldots, u_{(n-1) n}\right], \ldots, F\left[u_{1 n}, \ldots, u_{(n-1) n}\right]$ não são álgebras de polinômios pois as variáveis $u_{i j}$ 's satisfazem (3.26). Além disso, a álgebra livre $F\left\langle u_{1}\right\rangle$ torna-se a álgebra de polinômios $F\left[u_{1}\right]$ pois as potências de $u_{1}$ comutam quando agem sobre os bimódulos irredutíveis de Cayley, isto bem do seguinte resultado conhecido $\left[Z_{a}, \mathcal{A}_{c}\right]=0$.

No caso geral, usando os processos dos dois últimos casos de acima, não é difícil mostra 
que se $V=V_{a}^{m} \oplus V_{c}^{n}=\sum_{i=1}^{m} u_{i} \mathcal{L}+\sum_{j=1}^{n}$ Cay $_{j}$ temos a seguinte expressão da álgebra alternativa tensorial

$$
\begin{aligned}
\mathcal{L}[V] \cong & M_{2}\left(Q F\left[u_{i j}\right]\right)+\bar{Q} F\left[u_{i j}\right] \mathrm{Cay}_{1,2}+\bar{Q} F\left[u_{13}, u_{23}, \ldots, u_{1 n}, \ldots, u_{(n-1) n}\right] \mathrm{Cay}_{3} \\
& +\bar{Q} F\left[u_{14}, u_{24}, u_{34}, \ldots, u_{1 n}, \ldots, u_{(n-1) n}\right] \mathrm{Cay}_{4}+\ldots+\bar{Q} F\left[u_{1 n}, \ldots, u_{(n-1) n}\right] \mathrm{Cay}_{n}
\end{aligned}
$$

onde $Q=F\left\langle u_{1}, \ldots, u_{m}\right\rangle$ vira a álgebra polinomial $\bar{Q}=F\left[u_{1}, \ldots, u_{m}\right]$ quando age sobre os bimódulos irredutíveis de Cayley pois para quaisquer $u_{k}, u_{l} \in Z_{a}$ e $m_{i} \in \mathrm{Cay}_{i}$, então pelos resultados $\left(Z_{a}, \mathcal{A}, \mathcal{A}\right)=\left[Z_{a}, \mathcal{A}_{c}\right]=0$ do Lema 10 e pela $\mathbb{Z}_{2}$-graduação de $\mathcal{A}$, tem-se

$$
\begin{aligned}
{\left[u_{k}, u_{l}\right] m_{i} } & =\left(u_{k} u_{l}\right) m_{i}-\left(u_{l} u_{k}\right) m_{i} \\
& =u_{k}\left(u_{l} m_{i}\right)-u_{l}\left(u_{k} m_{i}\right) \\
& =u_{k}\left(m_{i} u_{l}\right)-\left(u_{k} m_{i}\right) u_{l} \\
& =u_{k}\left(m_{i} u_{l}\right)-u_{k}\left(m_{i} u_{l}\right) \\
& =0,
\end{aligned}
$$

Então, multiplicando a igualdade anterior pelo elemento $m_{j} \in \mathrm{Cay}_{j} \operatorname{com} i, j \neq$, obtemos a relação

$$
\left[u_{k}, u_{l}\right] u_{i j}=0
$$

Além disso, se fazemos as seguintes denotações $P \equiv Q F\left[u_{i j}\right]$ para $k=1, \ldots, m$ e $i \neq j=$ $1, \ldots, n$, e as álgebras $\bar{P}_{0} \equiv Q F\left[u_{i j}\right]=F\left[u_{k}, u_{i j}\right]$,

$$
\begin{gathered}
\bar{P}_{1} \equiv Q F\left[u_{13}, u_{23}, \ldots, u_{1 n}, \ldots, u_{(n-1) n}\right]=F\left[u_{k}, u_{13}, u_{23}, \ldots, u_{1 n}, \ldots, u_{(n-1) n}\right], \\
\bar{P}_{2} \equiv Q F\left[u_{14}, u_{24}, u_{34}, \ldots, u_{1 n}, \ldots, u_{(n-1) n}\right]=F\left[u_{k}, u_{14}, u_{24}, u_{34}, \ldots, u_{1 n}, \ldots, u_{(n-1) n}\right]
\end{gathered}
$$

$\mathrm{e}$

$$
\bar{P}_{n-2} \equiv Q F\left[u_{1 n}, \ldots, u_{(n-1) n}\right]=F\left[u_{k}, u_{1 n}, \ldots, u_{(n-1) n}\right]
$$

Temos

$$
\mathcal{L}[V] \cong M_{2}(P) \oplus \bar{P}_{0} \mathrm{Cay}_{1,2} \oplus \bar{P}_{1} \mathrm{Cay}_{3} \oplus \bar{P}_{2} \mathrm{Cay}_{4} \oplus \ldots \oplus \bar{P}_{n-2} \mathrm{Cay}_{n}
$$

É claro que a álgebra $\mathcal{L}[V]$ de $(3.31)$ não esta imerso na álgebra de Cayley-Dickson split $\mathbb{O}$ pois contém $M_{2}(P)$, onde $P$ é associativa mas não é comutativa.

Especificamente, denote $R_{n, m}=S_{n} \otimes_{F} F\left\langle Z_{m}\right\rangle / J$, onde $J$ é o ideal gerado pelo conjunto $\left\{u_{i j}\left[u_{k}, u_{l}\right] \mid 1 \leq i<j \leq n, 1 \leq k<l \leq m\right\}$, e seja $S_{n, m}=S_{n} \otimes_{F} F\left[Z_{m}\right]$. Então, por (3.31), a álgebra

$$
M_{2}\left(R_{n, m}\right)+\sum_{1}^{n} S_{n, m} V_{i}
$$

é isomorfa à álgebra tensorial $\mathcal{L}[V]$.

\subsection{Respostas do problema de Nathan Jacobson}

Portanto, de acordo aos três casos de acima, as respostas ao problema de N. Jacobson, sem qualquer restrição de dimensão e característica, são os seguintes: 
Teorema 24 (O caso associativo) A álgebra $M_{2}\left(F\left\langle Z_{m}\right\rangle\right)$ é isomorfa à álgebra tensorial $M_{2}(F)\left[\operatorname{Reg}_{1} \oplus \cdots \oplus \operatorname{Reg}_{m}\right]$.

Teorema 25 (O caso puramente Cayley) A subálgebra $M_{2}\left(S_{n}\right)+\sum_{1}^{n} S_{n} V_{i}$ da álgebra $\mathbb{O}\left(\mathbb{Q} \otimes_{F} F\left[X_{n} \cup Y_{n}\right]\right.$, é isomorfa à álgebra tensorial $M_{2}(F)\left[C a y_{1} \oplus \cdots \oplus C a y_{n}\right]$.

Teorema 26 (O caso geral) A álgebra $M_{2}\left(R_{n, m}\right) \oplus \sum_{1}^{n} S_{n, m} V_{i}$ é isomorfa à álgebra tensorial $M_{2}(F)\left[\operatorname{Cay}_{1} \oplus \cdots \oplus \operatorname{Cay}_{n} \oplus \operatorname{Reg}_{1} \oplus \cdots \oplus \operatorname{Reg}_{m}\right]$.

Corolário 27 Seja $\mathcal{A}$ uma $M_{2}(F)$-álgebra, $\mathcal{A}=M_{2}(F) \oplus V$. Então, $\mathcal{A}=\phi\left(M_{2}(F)[V]\right)$, onde $\phi: M_{2}(F)[V] \longrightarrow \mathcal{A}$ é o epimorfismo de $M_{2}(F)$-álgebras que estende a aplicação identidade $i d_{V}: V \longrightarrow V$.

Especificamente, $V=V_{a} \oplus V_{c}$, onde $V_{a}=\bigoplus_{k=1}^{m} c_{k} M_{2}(F), c_{k} \in Z_{a}, V_{c}=\bigoplus_{i=1}^{n}$ Cay, Cay $_{i} \cdot$ Cay $_{j}=b_{i j} M_{2}(F), b_{i j} \in Z(\mathcal{A})$. Sejam $\bar{S}_{n}=\operatorname{alg}\left\langle b_{i j} \mid 1 \leq i<j \leq n\right\rangle$ e $\bar{R}_{n, m}=\bar{S}_{n}\left\langle c_{k}\right| 1 \leq$ $k \leq m\rangle$, respectivamente, subálgebras de $Z(\mathcal{A})$ e $Z_{a}$. Denote também $\bar{c}_{k}=\left.c_{k}\right|_{V_{c}}$, e considere $\bar{S}_{n, m}=\bar{S}_{n}\left[\bar{c}_{k} \mid 1 \leq k \leq m\right]$; então $\mathcal{A}=M_{2}\left(\bar{R}_{n, m}\right) \oplus\left(\sum_{i=1}^{n} \bar{S}_{n, m} C_{a y}\right)$.

Corolário 28 Se $n=1$, então $\mathcal{A}=M_{2}(\mathcal{B}) \oplus$ Cay, onde $\mathcal{B}$ é associativa, Cay ${ }^{2}=0$ e $[\mathcal{B}$, Cay $]=[\mathcal{B}, \mathcal{B}]$ Cay $=0$.

Corolário 29 Se pelo menos um dos $b_{i j}$ no corolário 27 é invertível. Então, $n=2, \bar{R}_{2, m} \subseteq$ $Z(\mathcal{A})$ e $\mathcal{A} \subseteq \mathbb{O} \otimes_{F} \bar{R}_{2, m}$

Demonstração: Suponhamos que $b_{i j}$ é invertível. De (3.30), temos

$$
\left[c_{k}, c_{l}\right] b_{i j}=0
$$

Entao, $\left[c_{k}, c_{l}\right]=\left[c_{k}, c_{l}\right] b_{i j} b_{i j}^{-1}=0$. Portanto, $\bar{R}_{n, m} \subseteq Z(\mathcal{A})$. Também, de (3.28)

$$
\mathrm{Cay}_{k} \subseteq b_{i j}^{-1} b_{i k} \mathrm{Cay}_{j}+b_{i j}^{-1} b_{j k} \mathrm{Cay}_{i} .
$$

Assim, todas as componentes irredutíveis de $V_{c}$ com índices diferentes de $i, j$, estão contidos em $\mathrm{Cay}_{i} \oplus \mathrm{Cay}_{j}$. Isto implica que, $n=2$. Além disso, segue-se $\mathcal{A} \subseteq \mathbb{O} \otimes_{F} \bar{R}_{2, m}$

\section{Q.E.D.}

Exemplo 20 Seja $T_{2}=\left\{\left[\begin{array}{ll}\alpha & \beta \\ 0 & \gamma\end{array}\right] \mid \alpha, \beta, \gamma \in F\right\}$ e considere $R=T_{2}[x] / I$, onde $I$ é o ideal gerado pelo conjunto $\left\{x^{2}, x e_{12}\right\}$. Denote $\bar{x}=x+I \in R$, então

$$
\mathcal{A}=M_{2}(R) \oplus F\left[\bar{x}, e_{11}, e_{22}\right]\left(\operatorname{Cay}_{1} \oplus \operatorname{Cay}_{2}\right)
$$

com $b_{12}=\bar{x}$, é uma $M_{2}(F)$-álgebra alternativa não-associativa de dimensão 36. Além disso, $\mathcal{A}$ é a imagem homomórfica da álgebra tensorial $M_{2}(F)\left[\operatorname{Reg}_{1} \oplus \operatorname{Reg}_{2} \oplus \operatorname{Reg}_{3} \oplus \operatorname{Cay}_{1} \oplus \mathrm{Cay}_{2}\right]$, $\operatorname{com} \bar{S}_{2}=F[\bar{x}], \bar{R}_{3,2}=R, \bar{S}_{3,2}=F\left[\bar{x}, e_{11}, e_{22}\right]$.

\section{5 Álgebras e bimódulos alternativos com involução}

Seja $\mathcal{A}$ um álgebra alternativa com involução - . Lembremos que um bimódulo alternativo com involução sobre $\mathcal{A}$, é um bimódulo alternativo $V$ junto com um endomorfismo $v \longmapsto v^{*}$ de $V$ de período 2 , tal que,

$$
a \oplus v \longmapsto \bar{a} \oplus v^{*}
$$


é uma involução da extensão cisão nula $E=\mathcal{A} \oplus V$. O endomorfismo $v \longmapsto v^{*}$ de $V$, é chamada a involução de $V$.

Vamos denotar por $(D,-)$ uma álgebra com composição $D$ com involução -.

Exemplo 21 Seja Reg D o bimódulo regular de D. Então, os bimódulos regulares com involução Reg $D$ e-Reg D, são obtidas tomando o bimódulo regular Reg com a usual involução simplética ou seu negativo, isto é, os endomorfismos $a \longmapsto \bar{a} e a \longmapsto-\bar{a}$ são as involuções de $\operatorname{Reg} D$ e-Reg $D$, respectivamente.

Exemplo 22 Seja CayD o bimódulo de Cayley sobre D. Então, os bimódulos de Cayley com involução CayD e-CayD, são obtidas de maneira semelhante da involução usual (chamada-I), induzida na álgebra de Cayley-Dickson $C(u, \beta, \gamma)$ no processo de duplicação de Cayley-Dickson [19], isto é, os endomorfismos $m \longmapsto-m$ e $m \longmapsto m$ são as involuções de Cay $D$ e-Cay D, respectivamente. O bimódulo de Cayley Cay D é alternativo se, e somente se, $D$ é associativo.

Um caso particular dos exemplos 21 e 22, é o seguinte:

Exemplo 23 Se $D=M_{2}(F)$ com a involução simplética. Então, temos os seguintes bimódulos irredutiveis não isomorfos:

$$
\operatorname{Reg} D, \quad-\operatorname{Reg} D \quad \text { e } \operatorname{Cay} D \text {. }
$$

Observe que o bimódulo - Cay D neste caso, é não alternativo. No entanto, o bimódulo Cay D é alternativo não-associativo.

Consideremos o seguinte resultado provado por K. MacCrimmon [1] (veja o Teorema 9 do Cap.VII):

Teorema 30 (1) Qualquer bimódulo alternativo unitário com involução sobre uma álgebra de composição $(D,-)$ é a soma das imagens homomórficas dos quatro bimódulos Reg D, - Reg D, Cay D, -Cay D, onde os últimos dois podem ser retirados se D for não-associativo. (2) Qualquer bimódulo alternativo unitário com involução sobre $(D,-)$ é completamente redutivel e qualquer bimódulo irredutivel é isomorfo a uma componente irredutivel de Reg D, - Reg D, Cay D ou - Cay D se D for associativo e a uma componente irredutivel de Reg D ou -Reg D se D for não-associativo (portanto uma álgebra de octônios).

Voltando ao problema de Jacobson, suponhamos que a álgebra alternativa $\mathcal{A}$ tem uma involução $*$ que estende a involução simplética de $\mathbb{H} \cong M_{2}(F)$, isto é, * restrita a $\mathbb{H}$, é a involução simplética de $\mathbb{H}$, tal que, para todo $x \in \mathcal{A}$

$$
x+x^{*}=t(x) 1, \quad x x^{*}=x^{*} x=n(x) 1 ;
$$

onde $t(x) \in F$ e $n(x) \in F$ são o traço e a norma de $x$, respectivamente.

Ao considerarmos a álgebra alternativa $\mathcal{A}$ como um bimódulo alternativo unitário com involução * sobre $\mathbb{H}$, temos pelo Teorema 30, a decomposição

$$
\mathcal{A}=\sum_{i} \oplus \operatorname{Reg}_{i} \mathbb{H} \dot{+} \sum_{j} \oplus\left(-\operatorname{Reg}_{j} \mathbb{H}\right) \dot{+} \sum_{k} \oplus \operatorname{Cay}_{k} \mathbb{H} \dot{+} \sum_{l} \oplus\left(-\operatorname{Cay}_{l} \mathbb{H}\right)
$$

Isto implica, que o bimódulo $\mathcal{A}$ contêm dois conjuntos de elementos $\left\{u_{i}\right\}$ (as imagens de 1 no isomorfismo com Reg) e $\left\{v_{j}\right\}$ (as imagens de 1 no isomorfismo com -Reg), tais que, 
$\operatorname{Reg}_{i} \simeq u_{i} \mathbb{H}$ e $-\operatorname{Reg}_{j} \simeq v_{j} \mathbb{H}$. Dos isomorfismos, é claro que $u_{i}, v_{j} \in Z_{a}$ e por [1] (veja o Lema 2 do Cap.VII)

$$
u_{i}^{*}=u_{i} \quad \text { e } \quad v_{j}^{*}=-v_{j} \text { para todo } i, j .
$$

Além disso, $\mathrm{Cay}_{k}=F \cdot m_{1}^{(k)}+F \cdot m_{2}^{(k)} \mathrm{e}-\mathrm{Cay}_{l}=F \cdot n_{1}^{(l)}+F \cdot n_{2}^{(l)}$ são os bimódulos de Cayley irredutíveis de dimensão 2 sobre $\mathbb{H}$. Logo, de 3.32, temos

$$
\mathcal{A}=\sum_{i} \oplus u_{i} \mathbb{H} \dot{+} \sum_{j} \oplus v_{j} \mathbb{H} \dot{+} \sum_{k} \oplus \mathrm{Cay}_{k} \mathbb{H} \dot{+} \sum_{l} \oplus\left(-\mathrm{Cay}_{l} \mathbb{H}\right)
$$

$\operatorname{Sejam} \operatorname{Sym}(\mathcal{A})=\left\{x \in \mathcal{A} \mid x^{*}=x\right\}$ e $\operatorname{Skew}(\mathcal{A})=\left\{x \in \mathcal{A} \mid x^{*}=-x\right\}$ os espaços dos elementos simétricos e antissimétricos de $\mathcal{A}$, respectivamente.

Se $u \in Z_{a}$ e $a, b \in \mathbb{H}$, então $t(u) 1=u^{*}+u \in \operatorname{Sym}(\mathcal{A})$ e

$$
0=(t(u) 1, a, b)=\left(u^{*}+u, a, b\right)
$$

$\operatorname{logo}\left(u^{*}, a, b\right)=-(u, a, b)=0$. Também

$$
\left[u^{*}, a\right]=[t(u) 1-u, a]=[u, a]=0
$$

assim, $Z_{a}$ é invariante sobre a involução $*$. Portanto

$$
Z_{a}=\left(\operatorname{Sym}(\mathcal{A}) \cap Z_{a}\right) \oplus\left(\operatorname{Skew}(\mathcal{A}) \cap Z_{a}\right) .
$$

Pela Proposição 16 sabemos que o $\mathbb{H}$-bimódulo de Cayley $\mathcal{A}_{c}$ coincide com o subespaço $(\mathcal{A}, \mathbb{H}, \mathbb{H})$. Assim, ao considerar $a, b \in \mathbb{H}$ tais que $\alpha=[a, b]^{2} \neq 0$; então $1, a, b, a b$ formam uma base de $\mathbb{H}$ sobre $F$. Portanto, na prova da Proposição 16 (dada no Lema 12 de [16]) tem-se $\mathcal{A}_{c}=(\mathcal{A}, a, b) ; \operatorname{logo}$

$\mathcal{A}_{c}^{*}=(\mathcal{A}, a, b)^{*} \subseteq((\mathcal{A} a) b)^{*}-(\mathcal{A}(a b))^{*}=\bar{b}\left(\bar{a} \mathcal{A}^{*}\right)-(\bar{b} \bar{a}) \mathcal{A}^{*}=\left(\bar{b}, \bar{a}, \mathcal{A}^{*}\right) \subseteq(\mathbb{H}, \mathbb{H}, \mathcal{A})=\mathcal{A}_{c}$

e $\mathcal{A}_{c}$ é invariante sobre a involução *. Portanto

$$
\mathcal{A}_{c}=\left(\operatorname{Sym}(\mathcal{A}) \cap \mathcal{A}_{c}\right) \oplus\left(\operatorname{Skew}(\mathcal{A}) \cap \mathcal{A}_{c}\right) .
$$

Seja $\mathfrak{M}$ uma variedade de álgebras e consideremos um bimódulo $V$ para $\mathcal{A}$ na classe $\mathfrak{M}$. Se $E=\mathcal{A} \oplus V$ é a extensão cisão nula de $\mathcal{A}$ pelo bimódulo $V$, então definimos o núcleo $N(V)$ do bimódulo $V$, como a interseção do núcleo da extensão cisão nula com o bimódulo, isto é, $N(V)=E \cap V$.

Seja $w=(v, a, b) \in N\left(\mathcal{A}_{c}\right)$ para algum $v \in \mathcal{A}$. Então, por $(3.18)$

$$
0=(w, a, b)=((v, a, b), a, b)=[a, b](v, a, b),
$$

$\operatorname{logo} \alpha w=[a, b]^{2}(v, a, b)=[a, b]([a, b](v, a, b))=0$ e assim $w=0$. Portanto $N\left(\mathcal{A}_{c}\right)=0$.

Suponhamos que a involução $*$ de $\mathcal{A}$ é de tipo nuclear, isto é, todos os elementos simétricos estão contidos no núcleo (o centro associativo) de $\mathcal{A}, N(\mathcal{A})$. Agora, em $\mathcal{A}_{c}$ precisamos que os elementos simétricos estejam no núcleo. Como o núcleo de $\mathcal{A}_{c}$ é 0 , a involução $*$ aplica cada elemento de $\mathcal{A}_{c}$ no seu negativo, isto é, $m^{*}=-m$ para todo $m \in \mathcal{A}_{c}$. Assim, como para todo $m \in-$ Cay tem-se $m^{*}=m$, então $m=0$. Logo, - Cay $=0$.

Como $Z_{a}=\left(\operatorname{Sym}(\mathcal{A}) \cap Z_{a}\right) \oplus\left(\operatorname{Skew}(\mathcal{A}) \cap Z_{a}\right)$. Seja $v \in \operatorname{Skew}(\mathcal{A}) \cap Z_{a}$, isto é, $v^{*}=-v$ e $a v=v a$ para todo $a \in \mathcal{A}$. Lembre-se que na prova do Lema 10 temos mostrado que $\left[Z_{a}, \mathcal{A}_{c}\right]=0$. Assim, ao considerar o elemento $s=v m_{2}^{(k)}$, onde $m_{2}^{(k)}$ é um dos elementos da 
base de $\mathrm{Cay}_{k}$, obtemos

$$
s^{*}=\left(v m_{2}^{(k)}\right)^{*}=\left(m_{2}^{(k)}\right)^{*} v^{*}=\left(-m_{2}^{(k)}\right)(-v)=m_{2}^{(k)} v=v m_{2}^{(k)}=s ;
$$

portanto $s \in N\left(\mathcal{A}_{c}\right)=0$ e $u m_{2}^{(k)}=0$, o qual, implica facilmente que $v=0$. Portanto, Skew $(\mathcal{A}) \cap Z_{a}=0$ e $Z_{a}$ é a subálgebra dos elementos $u \in \mathcal{A}$, tais que, $a u=u a$ e $u^{*}=u$ para todo $a \in \mathcal{A}$. Consequentemente, como $v_{j}^{*}=-v_{j}$, então $v_{j}=0$ para todo $j$. Logo, $-\operatorname{Reg}=0$.

Assim, podemos retirar os bimódulos - Reg e - Cay da descomposição de $\mathcal{A}$, isto é,

$$
\mathcal{A}=\sum_{i} \oplus u_{i} \mathbb{H} \dot{+} \sum_{k} \oplus \mathrm{Cay}_{k}
$$

Portanto, se a álgebra alternativa $\mathcal{A}$ tem uma involução de tipo nuclear $x \mapsto x^{*}$, que estende a involução simplética de $\mathbb{H}$, então obtemos os resultados da seção 3.4. 


\section{Capítulo 4}

\section{Teoremas de Fatorização de Kronecker para as superálgebras alternativas}

H. M. Wedderburn mostrou que se uma álgebra associativa unitária $\mathcal{A}$ contém uma subálgebra simples central de dimensão finita $\mathcal{B}$ com o mesmo elemento identidade, então $\mathcal{A}$ é isomorfa a um produto de Kronecker $S \otimes_{F} \mathcal{B}$, onde $S$ é a subálgebra dos elementos que comutam com cada $b \in \mathcal{B}$. Em particular, se $\mathcal{A}$ contém $M_{n}(F)$ como subálgebra com o mesmo elemento identidade, temos $\mathcal{A} \cong S \otimes_{F} M_{n}(F)$ onde $S$ é a subálgebra de elementos que comutam com cada matriz unitária $e_{i j}$. Esse último resultado, é chamado "O Teorema de Coordenatização de Wedderburn" como tínhamos observado no capítulo 2. Kaplansky no Teorema 2 de [4] estendeu o resultado de Wedderburn para as álgebras alternativas $\mathcal{A}$ e $\mathcal{B}$ a álgebra de Cayley split. Jacobson no Teorema 1 de [2] deu uma nova prova do resultado de Kaplansky usando sua classificação dos bimodulos alternativos completamente redutíveis sobre corpos de característica diferente de 2. Esse resultado é conhecido como o Teorema de Fatorização de Kronecker de N. Jacobson.

No caso das superálgebras, M. López Díaz e I. Shestakov [7, 8] estudaram as representações das superálgebras alternativas e de Jordan simples excepcionais de característica 3 e por meio dessas representações, obtiveram uns análogos do Teorema de Fatorização de Kronecker para essas superálgebras. Similarmente, C. Martinez e E. Zelmanov [9] obtiveram um Teorema de Fatorização de Kronecker para a superálgebra de Kac excepcional $K_{10}$ de dimensão 10.

Neste capítulo, provamos um Teorema de Fatorização de Kronecker para as superálgebras alternativas que contém a superálgebra associativa $M_{(1 \mid 1)}(F)$ com o mesmo elemento identidade. Como corolário, respondemos ao análogo do problema de N. Jacobson [2] (veja o Cap. 3) para as superálgebras alternativas, isto é, descrevemos a cada superálgebra alternativa que contém a superálgebra associativa $M_{(1 \mid 1)}(F)$ com o mesmo elemento identidade sobre corpos de característica diferentes de 2.

Além disso, provamos o Teorema de Fatorização de Kronecker de N. Jacobson que funciona para o caso geral. Também, provamos um Teorema de Fatorização de Kronecker para as superálgebras alternativas, cuja parte par contém $\mathbb{O}$ com o mesmo elemento identidade.

M. Trushina e I. Shestakov [17] estudaram as representações das superálgebras alternativa simples $\mathbb{O}(4 \mid 4)$ ou $\mathbb{O}[u]$. Eles mostraram que cada bimódulo alternativo irredutível não-associativo fiel sobre essas superálgebras são isomorfos ao bimódulo regular ou a seu oposto das superálgebras $\mathbb{O}(4 \mid 4)$ ou $\mathbb{O}[u]$. Finalmente, provamos a redutibilidade completa dos bimódules sobre essas superálgebras e provamos alguns análogos do Teorema de Fatorização de Kronecker para cada superálgebra alternativa que contenha $\mathbb{O}(4 \mid 4)$ ou $\mathbb{O}[u]$ com o mesmo elemento identidade. 


\subsubsection{Superálgebras alternativas}

Lembremos, uma superálgebra é uma álgebra $\mathbb{Z}_{2}$-graduada $\mathcal{A}=\mathcal{A}_{\overline{0}} \oplus \mathcal{A}_{\overline{1}}$, onde $\mathcal{A}_{\overline{0}} \mathrm{e}$ $\mathcal{A}_{\overline{1}}$ são espaços de $\mathcal{A}$ tais que $\mathcal{A}_{i} \mathcal{A}_{j} \subseteq \mathcal{A}_{i+j}$ para todo $i, j \in \mathbb{Z}_{2}$. Os são espaços $\mathcal{A}_{\overline{0}}$ e $\mathcal{A}_{\overline{1}}$ são ditos como a parte par e ímpar, respectivamente, da $\mathbb{Z}_{2}$-graduação de $\mathcal{A}$.

Como antes, $\mathbb{H}$ denota a generalizada álgebra dos quaterniões.

Exemplo 24 A álgebra de Cayley-Dickson $\mathbb{O}=\mathbb{H}+v \mathbb{H}\left(v^{2}=\alpha \neq 0\right)$ tem a estrutura natural de uma álgebra $\mathbb{Z}_{2}$-graduada, onde a generalizada álgebra dos quaterniões $\mathbb{H}$ é a parte par e o $\mathbb{H}$-bimódulo de Cayley $v \mathbb{H}$ é a parte impar da $\mathbb{Z}_{2}-$ graduação de $\mathbb{O}$. Se $\mathbb{H} \cong M_{2}(F)$, temos a álgebra de Cayley-Dickson split e se $\mathbb{H}$ é de divisão, temos a álgebra de CayleyDickson de divisão.

E como em [15]:

Exemplo 25 Seja $\Gamma$ a álgebra de Grassmann sobre um corpo $F$ gerada pelos elementos $1, e_{1}, \ldots, e_{n}, \ldots$ tais que

$$
e_{i}^{2}=0, \quad e_{i} e_{j}=-e_{j} e_{i}
$$

Os produtos

$$
1, \quad e_{i_{1}} e_{i_{2}} \ldots e_{i_{k}}, \quad i_{1}<i_{2}<\cdots<i_{k},
$$

formam uma base de $\Gamma$ sobre $F$ (consideremos 1 como o produto do conjunto vacio dos elementos $\left.e_{i}\right)$. Denote-se por $\Gamma_{\overline{0}}$ e $\Gamma_{\overline{1}}$ os espaços gerados pelos produtos de comprimento par e impar; então $\Gamma$ é a soma direta desses espaços,

$$
\Gamma=\Gamma_{\overline{0}} \oplus \Gamma_{\overline{1}},
$$

onde

$$
\Gamma_{i} \Gamma_{j} \subseteq \Gamma_{i+j(\bmod 2)}, \quad i, i \in\{\overline{0}, \overline{1}\}
$$

Em outras palavras, $\Gamma$ é uma álgebra $\mathbb{Z}_{2}$-graduada (ou superálgebra) sobre $F$.

Definição 7 Seja $\mathcal{V}$ uma variedade de álgebras sobre $F$ definida pelas identidades homogêneas (veja [19]). Uma superálgebra $\mathcal{A}=\mathcal{A}_{\overline{0}} \oplus \mathcal{A}_{\overline{1}}$ é uma $\mathcal{V}$-superalgebra se sua envolvente de Grassmann $\Gamma(\mathcal{A})=\Gamma_{\overline{0}} \otimes \mathcal{A}_{\overline{0}}+\Gamma_{\overline{1}} \otimes \mathcal{A}_{\overline{1}}$ pertence a $\mathcal{V}$. Em particular, $\mathcal{A}$ é uma superálgebra alternativa se, e somente se, $\mathcal{A}$ satisfaz as seguintes (super)identidades:

$$
\begin{gathered}
(x, y, z)+(-1)^{|y||z|}(x, z, y)=0, \\
(x, y, z)+(-1)^{|x||y|}(y, x, z)=0, \\
(a, a, x)=0,
\end{gathered}
$$

onde $a_{0} \in \mathcal{A}_{0}, x, y, z \in \mathcal{A}_{0} \cup \mathcal{A}_{1}$ e $|x|$ denota a paridade de um elemento homogêneo $x$ of $\mathcal{A}$ : $|x|=i$ se $x \in \mathcal{A}_{i}$.

Observe que uma superálgebra alternativa não é o mesmo como uma álgebra alternativa $\mathbb{Z}_{2}$-graduada: as superálgebras alternativas simples de característica 3 , obtidas por I. Shestakov [16] não são álgebras alternativas. 
Exemplo 26 A superálgebra de Grassmann $\Gamma$ é uma superálgebra comutativa, pois sua envolvente de Grassmann $\Gamma(\Gamma)=\Gamma_{\overline{0}} \otimes \Gamma_{\overline{0}}+\Gamma_{\overline{1}} \otimes \Gamma_{\overline{1}}$ é comutativa. Isto vem do fato de que $\Gamma$ satisfaz a seguinte identidade de comutatividade (or supercomutatividade) graduada

$$
a b=(-1)^{|a||b|} b a
$$

onde $a, b \in \Gamma_{\overline{0}} \cup \Gamma_{\overline{1}}$.

De aqui em adiante denotaremos por $\mathcal{A}$ a cada superálgebra alternativa sobre $F$. Denotaremos também à parte par de $\mathcal{A}$ por $\mathcal{B}$ e a parte ímpar de $\mathcal{A}$ por $M$. Observamos que $\mathcal{B}$ é uma subálgebra alternativa de $\mathcal{A}$ e $M$ é um $\mathcal{B}$-bimódulo alternativo.

C.T.C. Wall [18] mostrou que cada superálgebra associativa simples de dimensão finita sobre um corpo algebricamente fechado $F$ é isomorfa a umas das seguintes superálgebras associativas:

$$
\mathcal{A}=M_{(m \mid k)}(F), \quad \mathcal{B}=\left\{\left[\begin{array}{ll}
* & 0 \\
0 & *
\end{array}\right]\right\} \quad, \quad M=\left\{\left[\begin{array}{ll}
0 & * \\
* & 0
\end{array}\right]\right\}
$$

$\mathrm{e}$

$$
\mathcal{A}=Q(n)=\left\{\left[\begin{array}{ll}
a & b \\
b & a
\end{array}\right] ; \quad a, b \in M_{n}(F)\right\}, \quad \mathcal{B}=\left\{\left[\begin{array}{ll}
a & 0 \\
0 & a
\end{array}\right]\right\}, \quad M=\left\{\left[\begin{array}{ll}
0 & b \\
b & 0
\end{array}\right]\right\} .
$$

Lembremos que no caso quando o corpo é de característica 2 qualquer álgebra alternativa $\mathbb{Z}_{2}$ - graduada é também uma superálgebra alternativa.

As superálgebras alternativas simples de característica 2 foram classificadas por I. Shestakov [16]. Nesse caso apareceram superálgebras simples não-triviais nem associativas. Assim, sobre um corpo de característica 2, qualquer tal superálgebra é isomorfa a uma das seguintes duas superálgebras obtidas do processo de Cayley-Dickson:

Exemplo 27 A superálgebra de Cayley-Dickson $\mathbb{O}(4 \mid 4)=\mathbb{O}=\mathbb{H} \oplus v \mathbb{H}, v^{2}=\gamma \neq$ $0(\gamma \in F)$ com a $\mathbb{Z}_{2}$-graduação inducida pelo processo de duplicação de Cayley-Dickson aplicada a generalizada álgebra dos quaterniões $\mathbb{H}$, onde

$$
\begin{gathered}
(a b) v=b(a v), \\
v(a b)=(v b) a, \\
(v a)(v b)=\alpha b \bar{a} .
\end{gathered}
$$

para todo $a, b \in \mathbb{H}$.

Exemplo 28 A álgebra de Cayley-Dickson dobrada $\mathbb{O}[u]=F[u] \otimes_{F} \mathbb{O}=\mathbb{O} \oplus \mathbb{O} u$, onde $F[u]=F+F u, 0 \neq \gamma=u^{2} \in F$.

\subsubsection{Superimódulos alternativos}

Os superbimódulos alternativos estão definidos de maneira semelhante ao caso nãograduado. Um bimódulo $V=V_{0} \oplus V_{1}$ sobre uma superálgebra alternativa $\mathcal{A}=\mathcal{B} \oplus M$ é chamado um $\mathcal{A}$-superbimódulo alternativo se, a extensão cisão nula $\mathcal{A} \oplus V=\left(\mathcal{B} \oplus V_{0}\right) \oplus\left(M \oplus V_{1}\right)$ é uma superálgebra alternativa.

Como no caso das álgebras, o problema principal da teoria de representações de superálgebras, é a descrição dos superbimódulos irredutíveis. Para as álgebras alternativas, tais bimódulos estão sempre definidos sobre álgebras simples ou sobre uma soma direta de duas álgebras simples, e no último caso, o bimódulo é associativo. Depois de isso, outro fato importante é mostrar a redutibilidade completa dos superbimódulos. 
68 TEOREMAS DE FATORIZAÇÃO DE KRONECKER PARA AS SUPERÁLGEBRAS

ALTERNATIVAS

\subsection{Um análogo do problema de Nathan Jacobson para as superálgebras alternativas}

Sejam $\mathcal{A}=\mathcal{B} \oplus M$ uma superálgebra alternativa com identidade 1 sobre $F$ e $e_{i} \in \mathcal{B}$, $i=1, \ldots, n$ um sistema idempotentes ortogonais dois a dois, tais que, $e_{1}+\cdots+e_{n}=1$. Além disso, seja $\mathcal{A}=\sum \mathcal{A}_{i j}$ a decomposição de Peirce de $\mathcal{A}$ relativo a esse sistema de idempotentes $\left\{e_{i}\right\}$.

Assim, temos a seguinte generalização para superálgebras alternativas do Teorema 10.

Teorema 31 Seja $\mathcal{A}=\mathcal{B} \oplus M$ uma superálgebra alternativa com elemento identidade 1 que contém um sistema de idempotentes ortogonais dois a dois e não nulos $e_{1}, \ldots, e_{n}$ tais que $1=e_{1}+\cdots+e_{n}$. Suponha que para cada $i \neq j$ existem $a_{i j} \in \mathcal{A}_{i j}$ e $b_{j i} \in \mathcal{A}_{j i}$ tais que

$$
a_{i j} b_{j i}=e_{i} \quad \text { e } \quad b_{j i} a_{i j}=e_{j} .
$$

Então, se $n \geq 3, \mathcal{A}$ é uma superálgebra associativa.

Demonstração: Seja $\Gamma(\mathcal{A})=\mathcal{B} \otimes \Gamma_{\overline{0}}+M \otimes \Gamma_{\overline{1}}$ a envolvente de Grassmann da superálgebra $\mathcal{A}$. Como $\mathcal{A}$ é uma superálgebra alternativa, resulta que $\Gamma(\mathcal{A})$ é uma álgebra alternativa. Note

$$
\Gamma(\mathcal{A}) \supset \mathcal{B} \otimes \Gamma_{\overline{0}} \supseteq\left\{\tilde{e}_{i}:=e_{i} \otimes 1 ; i=1, \ldots, n\right\}
$$

onde $\tilde{e}_{i}^{2}=\left(e_{i} \otimes 1\right)^{2}=e_{i} \otimes 1=\tilde{e}_{i}, \tilde{e}_{i} \tilde{e}_{j}=\left(e_{i} \otimes 1\right)\left(e_{j} \otimes 1\right)=e_{i} e_{j} \otimes 1=0$ para $i \neq j \mathrm{e}$ $\sum_{i=1}^{n} \tilde{e}_{i}=\sum_{i=1}^{n}\left(e_{i} \otimes 1\right)=1 \otimes 1=1_{\Gamma(\mathcal{A})}$; assim, $\Gamma(\mathcal{A})$ contém um sistema de idempotentes ortogonais $\left\{\tilde{e}_{i}\right\}$. Além disso, pela hipótese, para cada $i \neq j$ existem $a_{i j} \in \mathcal{A}_{i j}$ e $b_{j i} \in \mathcal{A}_{j i}$ tais que $a_{i j} b_{j i}=e_{i}$ e $b_{j i} a_{i j}=e_{j}$, então

$$
\tilde{a}_{i j} \tilde{b}_{j i}=\left(a_{i j} \otimes 1\right)\left(b_{j i} \otimes 1\right)=a_{i j} b_{j i} \otimes 1=e_{i} \otimes 1=\tilde{e}_{i}
$$

$\mathrm{e}$

$$
\tilde{b}_{j i} \tilde{a}_{i j}=\left(b_{j i} \otimes 1\right)\left(a_{i j} \otimes 1\right)=b_{j i} a_{i j} \otimes 1=e_{j} \otimes 1=\tilde{e}_{j},
$$

onde $\tilde{a}_{i j}$ e $\tilde{b}_{j i}$ pertencem à decomposição de Peirce de $\Gamma(\mathcal{A})$ relativo ao idempotente $1_{\Gamma(\mathcal{A})}$. Então, como $n \geq 3, \Gamma(\mathcal{A})$ é uma álgebra alternativa que satisfaz as condições to Teorema 10. Portanto, $\Gamma(\mathcal{A})$ é uma álgebra associativa e, consequentemente, $\mathcal{A}$ é uma superálgebra associativa.

Q.E.D.

Corolário 32 Seja $\mathcal{A}=\mathcal{B} \oplus M$ uma superálgebra alternativa com elemento identidade que contém a superálgebra associativa $M_{(m \mid k)}(F)$ com o mesmo elemento identidade. Se $m+k \geq 3$, então $\mathcal{A}$ é uma superálgebra associativa $e$

$$
\mathcal{A} \cong M_{(m \mid k)}(F) \otimes_{F} Z \cong M_{(m \mid k)}(Z)
$$

onde $Z$ é uma superálgebra associativa; além disso,

$$
\mathcal{B}=\left(M_{(m \mid k)}(F)\right)_{\overline{0}} \otimes_{F} Z_{\overline{0}}+\left(M_{(m \mid k)}(F)\right)_{\overline{1}} \otimes_{F} Z_{\overline{1}}
$$

$e$

$$
M=\left(M_{(m \mid k)}(F)\right)_{\overline{1}} \otimes_{F} Z_{\overline{0}}+\left(M_{(m \mid k)}(F)\right)_{\overline{0}} \otimes_{F} Z_{\overline{1}} .
$$


Exemplo 29 Consideremos a superálgebra associativa $\mathcal{A}=M_{(m \mid k)}(\mathbb{C})$ sobre $\mathbb{R}$ tal que $\mathcal{A}$ contém $M_{(m \mid k)}(\mathbb{R})$, então

$$
\mathcal{A} \cong M_{(m \mid k)}(\mathbb{R}) \otimes_{\mathbb{R}} Z \cong M_{(m \mid k)}(\mathbb{R}) \otimes_{\mathbb{R}} \mathbb{C}
$$

onde $Z=Z_{\overline{0}} \oplus Z_{\overline{1}}=\mathbb{R}+i \mathbb{R}=\mathbb{C}$. Além disso,

$$
\mathcal{B}=\left[\begin{array}{cc}
M_{m}(\mathbb{R}) & M_{m \times k}(i \mathbb{R}) \\
M_{k \times m}(i \mathbb{R}) & M_{k}(\mathbb{R})
\end{array}\right] \quad \text { e } \quad M=\left[\begin{array}{cc}
M_{m}(i \mathbb{R}) & M_{m \times k}(\mathbb{R}) \\
M_{k \times m}(\mathbb{R}) & M_{k}(i \mathbb{R})
\end{array}\right]
$$

Corolário 33 Cada superbimódulo alternativo unitário sobre $M_{(m \mid k)}(F)(m+k \geq 3)$ é associativo.

Consequentemente, a partir do Corolário 32 surge naturalmente o análogo do problema de Nathan Jacobson [2] para as superálgebras alternativas quando $m+k=2$ :

Descrever as superálgebras alternativas que contém $M_{(1 \mid 1)}(F)$ com o mesmo elemento identidade.

\subsection{Teoremas de Fatorização de Kronecker}

Seja $F$ um corpo de característica arbitrária. O seguinte resultado é conhecido como o Teorema de Fatorização de Kronecker de Nathan Jacobson.

Teorema 34 Seja $\mathcal{A}$ uma álgebra alternativa com elemento identidade, tal que, $\mathcal{A}$ contém (1) com o mesmo elemento identidade. Então, $\mathcal{A} \cong Z \otimes_{F} \mathbb{O}$ para uma certa álgebra associativa e comutativa $Z$.

Demonstração: No caso $\operatorname{Car} F \neq 2$, o resultado foi mostrado por N. Jacobson [2]. Daremos outra prova que funciona para o caso geral.

Consideremos $\mathcal{A}$ como um $\mathbb{O}$-bimódulo alternativo unitário. Em particular, podemos considerar $\mathcal{A}$ como um $\mathbb{H}$-bimódulo alternativo unitário, então pela Proposição 16

$$
\mathcal{A}=\mathcal{A}_{a} \oplus \mathcal{A}_{c}
$$

onde $\mathcal{A}_{a}$ é um bimódulo associativo e $\mathcal{A}_{c}$ é um bimódulo de Cayley. Sabemos que $\mathcal{A}_{a}$ contém um conjunto de elementos $\left\{u_{i}\right\}$ (as imagens de 1 no isomorfismo com Reg $\mathbb{H}$ ), tal que, $\mathcal{A}_{a}=\sum u_{i} \mathbb{H}$, onde $u_{i} \in Z_{a}=\left\{a \in \mathcal{A}_{a} \mid[a, \mathbb{H}]=0\right\}$. Além disso, pelo Lema 10

$$
\left(Z_{a}, \mathcal{A}, \mathcal{A}\right)=\left[Z_{a}, \mathcal{A}_{c}\right]=0
$$

e, como $\mathcal{A}$ contem $\mathbb{O}$; em particular $\left(Z_{a}, Z_{a}, \mathbb{O}\right)=\left(Z_{a}, \mathbb{O}, \mathbb{O}\right)=0$.

Seja $\mathcal{B}=\sum u_{i} \mathbb{O}$. É claro que $\mathcal{B}$ é um $\mathbb{O}$-subbimódulo de $\mathcal{A}$ e

$$
\mathcal{B} \supseteq \sum u_{i} \mathbb{H}=\mathcal{A}_{a}
$$

Sabemos que a álgebra de Cayley-Dickson $\mathbb{O}$ tem a decomposição $\mathbb{H} \oplus v \mathbb{H}$, onde $v^{2}=\gamma \neq 0$ $(\gamma \in F)$ e por $(4.2)$

$$
(v x)(v y)=\gamma y \bar{x}
$$


para todo $x, y \in \mathbb{H}$. Escolha $a, b \in \mathbb{H}$, tais que, $\alpha=[a, b]^{2} \neq 0(\alpha \in F)$. Seja $w \in \mathcal{A}_{c}$, $\log 0$

$$
\begin{aligned}
\alpha \gamma w & =w(\gamma[a, b] \overline{[\bar{b}, \bar{a}]})=w((v[\bar{b}, \bar{a}])(v[a, b])) \\
& =(w \circ(v[\bar{b}, \bar{a}]))(v[a, b])-(v[\bar{b}, \bar{a}])(w(v[a, b])) .
\end{aligned}
$$

É claro que $v \mathbb{H}$ está contido em $\mathcal{A}_{c}$. Assim, pelo Lema 8 , sabemos que $\mathcal{A}_{c} \mathcal{A}_{c} \subseteq \mathcal{A}_{a}$, então $w \circ(v[\bar{b}, \bar{a}]) \in \mathcal{A}_{a}$ e $w(v[a, b]) \in \mathcal{A}_{a}$; portanto, $\alpha \gamma w \in \mathcal{B}$ e como $\alpha, \gamma \neq 0$, temos $w \in \mathcal{B}$. Logo, $\mathcal{A}_{c} \subseteq \mathcal{B}$. Então, $\mathcal{A}=\mathcal{B}$. Como cada $u_{i} \mathbb{O}$ é irredutível, $\mathcal{A}=\mathcal{B}$ é completamente redutível.

De $\left[Z_{a}, \mathcal{A}_{c}\right]=0$, temos $\left[Z_{a}, v \mathbb{H}\right]=0$ pois $v \mathbb{H}$ esta contido em $\mathcal{A}_{c}$. Então,

$$
\left[Z_{a}, \mathbb{O}\right]=0
$$

Como $\mathcal{A}=\sum u_{i} \mathbb{O}$, cada elemento de $\mathcal{A}$ pode ser escrito em uma única forma, da forma $\sum u_{i} a_{i}$ com $a_{i} \in \mathbb{O}$. Pelo Lema $9, Z_{a}$ é uma álgebra associativa. Por outro lado, se $x=$ $\sum u_{i} a_{i} \in Z_{a}, a_{i} \in \mathbb{O}$. Então, $a x=x a$ para todo $a \in \mathbb{O}$, isto é,

$$
\begin{aligned}
\sum a u_{i} a_{i} & =\sum u_{i} a_{i} a, \\
\sum u_{i} a a_{i} & =\sum u_{i} a_{i} a ;
\end{aligned}
$$

portanto $a a_{i}=a_{i} a$. Como $\mathbb{O}$ é central, isso, implica $a_{i}=\lambda_{i} 1$, onde $\lambda_{i} \in F$. Portanto, $Z_{a}$ é o espaço gerado pelos $u_{i}$, isto é, $Z_{a}=\sum F u_{i}$. Logo, é claro que $\mathcal{A}=Z_{a} \otimes_{F} \mathbb{O}$.

Fixemos elementos arbitrários $x, y \in Z_{a}$ e $a, b, c \in \mathbb{O}$. Aplicando a linearização de (3.18), temos

$$
\begin{aligned}
{[x, y](a, b, c)=} & -[a, y](x, b, c)-[x, b](a, y, c)-[a, b](x, y, c)+(x, y,(a, b, c)) \\
& +(a, y,(x, b, c))+(x, b,(a, y, c))+(a, b,(x, y, c))=0 .
\end{aligned}
$$

Podemos escrever o produto anterior como $[x, y] \otimes(a, b, c)=0$. Assim, como $\mathbb{O}$ é não associativa, existem $a, b, c \in \mathbb{O}$, tais que, $(a, b, c) \neq 0$. Então, $[x, y]=0$ e $\left[Z_{a}, Z_{a}\right]=0$; portanto, $Z_{a}$ é uma álgebra associativa e comutativa.

Se denotamos $Z=Z_{a}$, temos $\mathcal{A} \cong Z \otimes_{F} \mathbb{O}$.

Isso prova o Teorema.

Q.E.D.

Com a ajuda do Teorema 34, podemos classificar aos bimódulos alternativos unitários sobre a álgebra de Cayley-Dickson $\mathbb{O}$.

Teorema 35 Cada bimódulo alternativo unitário sobre (O) é completamente redutivel.

Demonstração: No caso $\operatorname{Car} F \neq 2$, o resultado foi mostrado por N. Jacobson [3]. Daremos outra prova que funciona para o caso geral.

Seja $V$ um bimódulo alternativo unitário sobre $\mathbb{O}$. A extensão cisão nula $E=\mathbb{O} \oplus V$ de (1) pelo bimódulo $V$ é uma álgebra alternativa que contém $\mathbb{O}$ como uma subálgebra unitária. Então, pela demonstração do Teorema 34, temos $E=\sum \oplus \operatorname{Reg} \mathbb{O}$. Portanto, $V=\sum \oplus \operatorname{Reg} \mathbb{O}$, isto mostra que, $V$ é completamente redutível.

Isto prova o Teorema.

Q.E.D.

A seguir, generalizamos o Teorema 34 para as superálgebras alternativas unitárias, mas para isso precisamos primeiro o seguinte resultado. 
Lema 17 Seja $\mathcal{A}=\mathcal{B} \oplus M$ uma superálgebra alternativa tal que $\mathcal{A}$ contém uma subsuperálgebra $U$ separável de dimensão finita. Consideremos $\Omega=\{a \in \mathcal{A}:[a, U]=0\}$, onde $[a, u]=a u-(-1)^{|a||u|} u a$ para todo $u \in U$. Então, $\Omega$ é uma subsuperálgebra de $\mathcal{A} e$

$$
(a u)(b v)=(-1)^{|u||b|}(a b)(u v)
$$

é válida para todo $a, b \in U$ e $u, v \in \Omega$.

Demonstração: Considere as envolventes de Grassmann $\Gamma(\mathcal{A})$ e $\Gamma(U)$, respectivamente de $\mathcal{A}$ e $U$. Assim, $\Gamma(\mathcal{A})$ é uma álgebra alternativa que contém uma subálgebra separável de dimensão finita $\Gamma(U)$. Portanto, pelo Lema 1 de [2], o conjunto

$$
\Theta=\{\widetilde{a} \in \Gamma(\mathcal{A}):[\widetilde{a}, \Gamma(U)]=0\}
$$

é uma subálgebra de $\Gamma(\mathcal{A})$. Além disso, $(\Gamma(\mathcal{A}), \Gamma(U), \Theta)=0$.

Vamos mostrar que $\Theta=\Gamma(\Omega)$. Seja $\widetilde{a}$ um elemento homogêneo de $\Theta$, isto é, $\widetilde{a}=g_{a} \otimes a$ onde $g_{a} \in \Gamma_{0} \cup \Gamma_{1}$ e $a \in \mathcal{A}_{0} \cup \mathcal{A}_{1}$ tem a mesma paridade. Então, para todo elemento homogêneo $\widetilde{x}=g_{x} \otimes x \in \Gamma(U)$

$$
0=[\widetilde{a}, \widetilde{x}]=g_{a} g_{x} \otimes\left(a x-(-1)^{|a||x|} x a\right)=g_{a} g_{x} \otimes[a, x]
$$

e como $g_{a} g_{x}$ é um elemento da base da álgebra de Grassmann, temos $[a, x]=0$ para todo $x \in U_{0} \cup U_{1}$. Logo, $a \in \Omega$; portanto $\widetilde{a} \in \Gamma(\Omega)$ e $\Theta \subseteq \Gamma(\Omega)$. Agora, seja $\widetilde{a}$ um elemento homogêneo de $\Gamma(\Omega)$, isto é, $\widetilde{a}=g_{a} \otimes a$ para $a \in \Omega_{0} \cup \Omega_{1}$. Então, $[a, x]=0$ para todo $x \in U_{0} \cup U_{1}$. Assim,

$$
[\widetilde{a}, \widetilde{x}]=\left[g_{a} \otimes a, g_{x} \otimes x\right]=g_{a} g_{x} \otimes[a, x]=0
$$

para todo $\widetilde{x} \in \Gamma(U)$. Logo, $\widetilde{a} \in \Theta$; portanto $\Gamma(\Omega) \subseteq \Theta$.

Assim, $(\Gamma(\mathcal{A}), \Gamma(U), \Gamma(\Omega))=0$. Voltando para as superálgebras, $\Omega$ é uma subsuperálgebra de $\mathcal{B}$ e a última igualdade implica $(\mathcal{A}, U, \Omega)=0$. Além disso, pelo Lema 1 de [2] $(\widetilde{a} \widetilde{u})(\widetilde{b} \widetilde{v})=$ $(\widetilde{a} \widetilde{b})(\widetilde{u} \widetilde{v})$ para to $\widetilde{a}, \widetilde{b} \in \Gamma(U)$ e $\widetilde{u}, \widetilde{v} \in \Gamma(\Omega)$, isto é, $\widetilde{a}=g_{a} \otimes a, \widetilde{b}=g_{b} \otimes b, \widetilde{u}=g_{u} \otimes u$, $\widetilde{u}=g_{v} \otimes v$

$$
\begin{aligned}
(\widetilde{a} \widetilde{u})(\widetilde{b} \widetilde{v}) & =(\widetilde{a} \widetilde{b})(\widetilde{u} \widetilde{v}) \\
\left(g_{a} g_{u} \otimes a u\right)\left(g_{b} g_{v} \otimes b v\right) & =\left(g_{a} g_{b} \otimes a b\right)\left(g_{u} g_{v} \otimes u v\right), \\
g_{a} g_{u} g_{b} g_{v} \otimes(a u)(b v) & =g_{a} g_{b} g_{u} g_{v} \otimes(a b)(u v),
\end{aligned}
$$

mas $g_{b} g_{u}=(-1)^{|b||u|} g_{u} g_{b}$. Então, $g_{a} g_{u} g_{b} g_{v} \otimes\left((a u)(b v)-(-1)^{|b||u|}(a b)(u v)\right)=0$ e, como $g_{a} g_{u} g_{b} g_{v}$ é um elemento da base de $\Gamma$, temos $(a u)(b v)=(-1)^{|b||u|}(a b)(u v)$ para todo $a, b \in U$ e $u, v \in \Omega$.

Isso prova o Lema.

Q.E.D.

No que segue, provamos um Teorema de Fatorização de Kronecker para as superálgebras alternativas, onde a parte par da superálgebra contém $\mathbb{O}$ com o mesmo elemento identidade.

Teorema 36 Seja $\mathcal{A}=\mathcal{B} \oplus M$ uma superálgebra alternativa, tal que, $\mathcal{B}$ contém $\mathbb{O}$ e ambos tem o mesmo elemento identidade de $\mathcal{A}$. Então, $\mathcal{A} \cong \Omega \otimes_{F} \mathbb{O}$ para uma certa superálgebra associativa e comutativa $\Omega$. 
Demonstração: Sabemos que $\mathcal{B}$ é uma subálgebra alternativa de $\mathcal{A}$ que contém $\mathbb{O}$ e ambos têm a mesma identidade de $\mathcal{A}$. Consideremos $\mathcal{B}$ como um $\mathbb{O}$-bimódulo alternativo unitário. Então, pelo Teorema $35, \mathcal{B}$ é completamente redutível, isto implica que, o bimódulo $\mathcal{B}$ contém um conjunto de elementos $\left\{u_{i}\right\}$ (as imagens de 1 nos isomorfismos com Reg $\mathbb{O}$ ), tais que, $\mathcal{B}=\sum u_{i} \mathbb{O}$ e cada elemento de $\mathcal{B}$ pode ser escrito em uma única forma, da forma $\sum u_{i} a_{i}$ onde $a_{i} \in \mathbb{O}$.

Além disso, como $\mathcal{A}$ é uma superálgebra alternativa, temos que, $M$ é um $\mathcal{B}$-bimódulo alternativo unitário; em paticular, $M$ é uma $\mathbb{O}$-bimódulo. Assim, como no paragrafo acima, o bimódulo $M$ contém um conjunto de elementos $\left\{v_{j}\right\}$ (as imagens de 1 nos isomorfismos com Reg $\mathbb{O}$, tais que, $M=\sum v_{j} \mathbb{O}$ e cada elemento de $M$ pode ser escrito em uma única forma, da forma $\sum v_{j} a_{j}$ onde $a_{j} \in \mathbb{O}$.

Seja $\Omega=\{x \in \mathcal{A} ;[x, \mathbb{O}]=0\}$. Pelo Lema $17, \Omega$ é uma subsuperálgebra e $(\mathcal{A}, \mathbb{O}, \Omega)=0$; em particular $(\Omega, \mathbb{O}, \Omega)=(\mathbb{O}, \mathbb{O}, \Omega)=0$. Claramente $u_{i}, v_{j} \in \Omega$. Por outro lado, seja $x=$ $\sum u_{i} a_{i}+\sum v_{j} a_{j} \in \Omega, a_{i}, a_{j} \in \mathbb{O}$. Então, $a x=x a$ para todo $a \in \mathbb{O}$, isto é,

$$
\begin{aligned}
& \sum a u_{i} a_{i}+\sum a v_{j} a_{j}=\sum u_{i} a_{i} a+\sum v_{j} a_{j} a, \\
& \sum u_{i} a a_{i}+\sum v_{j} a a_{j}=\sum u_{i} a_{i} a+\sum v_{j} a_{j} a .
\end{aligned}
$$

Portanto, $a a_{i}=a_{i} a$ e $a a_{j}=a_{j} a$. Como $\mathbb{O}$ é central, isso implica, $a_{i}=\lambda_{i} 1$ e $a_{j}=\lambda_{j} 1$, onde $\lambda_{i}$ e $\lambda_{j}$ estão no corpo $F$. Portanto, $\Omega$ é o espaço gerado pelos $u_{i}$ e os $v_{j}$, isto é,

$$
\Omega=\sum F u_{i} \oplus \sum F v_{j}
$$

Logo, é claro que $\mathcal{A}=\Omega \otimes_{F} \mathbb{O}$.

Fixemos elementos arbitrários $x, y, z \in \Omega$ e $a, b, c \in \mathbb{O}$. Como $\mathbb{O}$ contem $\mathbb{H}$, escolha elementos $a, b \in \mathbb{H}$ tais que $[a, b]^{2}=\alpha \in F, \alpha \neq 0$. Então, pela superlinearização de (3.18), obtemos

$$
\begin{aligned}
{[a, b](x, y, z)=} & \pm[a, y](x, b, z) \pm[x, b](a, y, z) \pm[x, y](a, b, z) \pm(a, b,(x, y, z)) \\
& \pm(a, y,(x, b, z)) \pm(x, b,(a, y, z)) \pm(x, y,(a, b, z))=0 .
\end{aligned}
$$

Similarmente, $(x, y, z)[a, b]=0$. Assim,

$$
\begin{aligned}
\alpha(x, y, z)=[a, b]^{2}(x, y, z)= & {[a, b]([a, b](x, y, z)) } \\
& \pm(([a, b](x, y, z))[a, b]-[a, b]((x, y, z)[a, b]))=0
\end{aligned}
$$

e $(x, y, z)=0$; portanto, $\Omega$ é associativa.

Aplicando novamente a superlinearização de (4.18), temos

$$
\begin{aligned}
{[x, y](a, b, c)=} & \pm[a, y](x, b, c) \pm[x, b](a, y, c) \pm[a, b](x, y, c) \pm(x, y,(a, b, c)) \\
& \pm(a, y,(x, b, c)) \pm(x, b,(a, y, c)) \pm(a, b,(x, y, c))=0 .
\end{aligned}
$$

Podemos escrever o produto anterior como $[x, y] \otimes(a, b, c)=0$. Assim, como $\mathbb{O}$ é não associativa, existem $a, b, c \in \mathbb{O}$, tais que, $(a, b, c) \neq 0$. Então, $[x, y]=0$ e $[\Omega, \Omega]=0$; portanto, $\Omega$ é uma superálgebra associativa e comutativa.

Isso prova o Teorema. 
Seja $\mathcal{A}$ uma arbitrária superálgebra e $U$ uma subsuperálgebra de $\mathcal{A}$. O conjunto

$$
C_{\mathcal{A}}(\mathcal{B})=\{a \in \mathcal{A} \mid[a, \mathcal{B}]=0\}
$$

é chamado o centralizador de $U$ em $\mathcal{A}$.

Usando a envolvente de Grassmann e a definição de centralizador, podemos dar uma outra demonstração do teorema 36:

Demonstração: Consideremos a envolvente de Grassmann $\Gamma(\mathcal{A})$ de $\mathcal{A}$. Identificando $\mathbb{O}$ com $1 \otimes \mathbb{O} \subseteq \Gamma_{0} \otimes \mathcal{B}$, concluímos, pelo Teorema 34 que, existe uma álgebra associativa e comutativa $\bar{Z}$ tal que $\Gamma(\mathcal{A}) \cong Z \otimes \mathbb{O}$, onde $Z$ é o centralizador de $\mathbb{O}$ em $\Gamma(\mathcal{A})$.

Denotemos por $\Omega$ o centralizador de $\mathbb{O}$ em $\mathcal{A}$. Seja $\widetilde{a}$ um elemento homogêneo de $Z$, isto é, $\widetilde{a}=g_{a} \otimes a$ onde $a \in \mathcal{A}$ é um elemento homogêneo, tal que, $[\widetilde{a}, x]=0$ para todo $x \in \mathbb{O}$. Como $x=1 \otimes x$, então $0=[\widetilde{a}, x]=\left[g_{a} \otimes a, 1 \otimes x\right]=g_{a} \otimes[a, x]$ e como $g_{a}$ é um elemento da base da álgebra de Grassmann, temos $[a, x]=0$ para todo $x \in \mathbb{O}$. Logo, $a \in \Omega$; portanto $\widetilde{a} \in \Gamma(\Omega)$ e $Z \subseteq \Gamma(\Omega)$. Agora, seja $\widetilde{a} \in \Gamma(\Omega)$, isto é, $\widetilde{a}=g_{a} \otimes a$ para $a \in \Omega$ homogêneo. Então,

$$
[\widetilde{a}, x]=\left[g_{a} \otimes a, 1 \otimes x\right]=g_{a} \otimes[a, x]=0
$$

para todo $x \in \mathbb{O}$. Logo, $\widetilde{a} \in Z$ e $\Gamma(\Omega) \subseteq Z$. Assim, $Z=\Gamma(\Omega)$, isto é, o centralizador de (1) na envolvente de Grassmann de $\mathcal{A}$ é a envolvente de Grassmann do centralizador de $\mathbb{O}$ em $\mathcal{A}$; portanto $\mathcal{A} \cong\left(\Omega_{0}+\Omega_{1}\right) \otimes_{F} \mathbb{O}$, onde $\Omega=\Omega_{0}+\Omega_{1}$ é uma superálgebra associativa e comutativa de $\mathcal{A}$.

Q.E.D.

A seguir, vamos supor que a característica do corpo $F$ é diferente de 2 .

N. A. Pisarenko [11] mostro que cada bimódulo alternativo unitário sobre $M_{(1 \mid 1)}(F)$ é associativo e completamente redutível e, os únicos $M_{(1 \mid 1)}(F)$-bimódulos irredutíveis são o bimódulo regular $\operatorname{Reg} M_{(1 \mid 1)}(F)$ e seu oposto.

Vamos provar para a superálgebra associativa unitária $M_{(1 \mid 1)}(F)$ um análogo do teorema de Fatorização de Kronecker de N. Jacobson [2].

Teorema 37 Seja $\mathcal{A}$ uma superálgebra alternativa tal que $\mathcal{A}$ contém $M_{(1 \mid 1)}(F)$ com o mesmo elemento identidade. Então, $\mathcal{A} \cong U \widetilde{\otimes}_{F} M_{(1 \mid 1)}(F)$ para uma certa superálgebra associativa $U$.

Demonstração: Considere $\mathcal{A}$ como um $M_{(1 \mid 1)}(F)$-bimódulo alternativo unitário. Por [11], temos $\mathcal{A}=\sum_{i} u_{i} M_{(1 \mid 1)}(F)$, onde $u_{i} \in Z_{a}(\mathcal{A})=\left\{u \in \mathcal{A}:\left[u, M_{(1 \mid 1)}(F)\right]=0\right\}$. Se $U=Z_{a}$. Então, $\mathcal{A}=U M_{(1 \mid 1)}(F)$ e mostraremos que $U$ é a superálgebra desejada.

Primeiro mostraremos que $U$ é uma subsuperálgebra de $\mathcal{A}$. Consideremos elementos arbitrários $u, v, w \in U$ e $a, b, c \in M_{(1 \mid 1)}(F)$. Assim, pelo fato que $\mathcal{A}$ é um $M_{(1 \mid 1)}(F)$-bimódulo associativo, isto é, $\left(\mathcal{A}, M_{(1 \mid 1)}(F), M_{(1 \mid 1)}(F)\right)=0$ e, pelas superlinearizações de (3.18) e (3.19), temos

$$
\begin{aligned}
{[a, b](u, v, c)=} & \pm\left[u_{i}, b\right](a, v, c) \pm[a, v](u, b, c) \pm[u, v](a, b, c) \pm(a, b,(u, v, c)) \\
& \pm(u, b,(a, v, c)) \pm(a, v,(u, b, c)) \pm(u, v,(a, b, c)) \\
= & \pm(a, b,(u, v, c)) \\
= & \pm((u, a, b), v, c) \pm(u,(v, a, b), c) \pm(u, v,(c, a, b)) \\
& \pm[v,(u, c,[a, b])] \pm([u, c], v,[a, b])=0 .
\end{aligned}
$$


Similarmente $(u, v, c)[a, b]=0$. Escolhendo elementos homogêneos $a, b \in M_{(1 \mid 1)}(F)$ tais que $[a, b]^{2}=\alpha \in F, \alpha \neq 0$. Então, usando a superidentidade que define a uma superálgebra alternativa, obtemos

$$
\begin{aligned}
\alpha(u, v, c)=[a, b]^{2}(u, v, c)= & {[a, b]([a, b](u, v, c)) } \\
& \pm(([a, b](u, v, c))[a, b]-[a, b]((u, v, c)[a, b]))=0
\end{aligned}
$$

e $(u, v, c)=0$. Assim, $\left(U, U, M_{(1 \mid 1)}(F)\right)=0$. Logo, pela seguinte identidade

$$
[x y, z]-x[y, z]-(-1)^{|y||z|}[x, z] y-3(x, y, z)=0
$$

que é válida em toda superálgebra alternativa (veja [15])

$$
[u v, c]= \pm u[v, c] \pm[u, c] v \pm 3(u, v, c)=0
$$

portanto $U U \subseteq U$

Além disso, de novo pela superlinearização de (3.18), temos

$$
\begin{aligned}
{[a, b](u, v, w)=} & \pm\left[u_{i}, b\right](a, v, w) \pm[a, v](u, b, w) \pm[u, v](a, b, w) \pm(a, b,(u, v, w)) \\
& \pm(u, b,(a, v, w)) \pm(a, v,(u, b, w)) \pm(u, v,(a, b, w))=0 .
\end{aligned}
$$

Similarmente $(u, v, w)[a, b]=0$. Logo,

$$
\begin{aligned}
\alpha(u, v, w)=[a, b]^{2}(u, v, w)= & {[a, b]([a, b](u, v, w)) } \\
& \pm(([a, b](u, v, w))[a, b]-[a, b]((u, v, w)[a, b]))=0
\end{aligned}
$$

o qual, implica $(u, v, w)=0$. Portanto, $U$ é associativa.

É claro que $\mathcal{A}$ é a imagem homomórfica de $U \widetilde{\otimes}_{F} M_{(1 \mid 1)}(F)$. Suponha que $w=u_{1} e_{11}+$ $u_{2} e_{22}+u_{12} e_{12}+u_{21} e_{21}=0$ para alguns $u_{i}, u_{i j} \in U$. Então, como $\mathcal{A}$ é $M_{(1 \mid 1)}(F)$-bimódulo associativo, temos

$$
\begin{aligned}
& 0=w e_{11}=u_{1} e_{11}+u_{21} e_{21} \\
& 0=w e_{22}=u_{2} e_{22}+u_{12} e_{12} .
\end{aligned}
$$

Multiplicar à primeira equação separadamente pela esquerda por $e_{11}$ e $e_{12}$, assim $0=u_{1} e_{11}$ e $0=u_{21} e_{11}$. Logo,

$$
\begin{gathered}
0=\left(e_{21}\left(u_{1} e_{11}\right)\right) e_{12}=u_{1} e_{22}, \\
0=\left(e_{21}\left(u_{21} e_{11}\right)\right) e_{12}=u_{21} e_{22} .
\end{gathered}
$$

Então,

$$
\begin{gathered}
0=u_{1} e_{11}+u_{1} e_{22}=u_{1} \\
0=u_{21} e_{11}+u_{21} e_{22}=u_{21} .
\end{gathered}
$$

Similarmente, de $0=w e_{22}=u_{2} e_{22}+u_{12} e_{12}$ segue $u_{2}=u_{12}=0$. Portanto, $\mathcal{B} \cong U \widetilde{\otimes}_{F} M_{(1 \mid 1)}(F)$. Isto prova o Teorema.

Q.E.D.

Corolário 38 Seja $\mathcal{A}$ uma superálgebra alternativa, tal que, $\mathcal{A}$ contém $M_{(1 \mid 1)}(F)$ com o mesmo elemento identidade. Então, $\mathcal{A}$ é uma superálgebra associativa.

O corolário 38 responde ao análogo do problema de N. Jacobson para as superálgebras alternativas sobre corpos de característica diferente de 2 . 
Observação 39 Se a característica do corpo $F$ for 2 , a superálgebra $M_{(1 \mid 1)}(F)$ possui um bimódulo de Cayley irredutível unitário que não é associativo (veja, [17]).

No que segue, vamos supor que a característica do corpo $F$ é igual a 2.

Maria Trushina e Ivan Shestakov [17] mostraram os seguintes resultados:

Teorema 40 Seja $V$ um bimódulo alternativo irredutivel fiel sobre a superálgebra $\mathbb{O}(4 \mid 4)$. Então, $V$ é isomorfo a Reg $\mathbb{O}(4 \mid 4)$ ou a seu oposto.

Teorema 41 Seja $V$ um bimódulo alternativo irredutivel fiel sobre a superálgebra $\mathbb{O}[u]$. Então, $V$ é isomorfo a $\operatorname{Reg} \mathbb{O}[u]$ ou a seu oposto.

Nos seguintes resultados vamos descrever a redutibilidade completa dos bimódulos alternativos unitários sobre as superálgebras alternativas simples $\mathbb{O}(4 \mid 4)$ e $\mathbb{O}[u]$. Dividimos as provas em uma sequencia de Lemas.

Seja $V$ um bimódulo alternativo unitário sobre a superálgebra $\mathbb{O}(4 \mid 4)=\mathbb{H}+M$, onde $M=v \mathbb{H}$. Pela Proposição $16, V=V_{a} \oplus V_{c}$, onde $V_{a}$ é um $\mathbb{H}$-bimódulo associativo unitário e $V_{c}$ é um $\mathbb{H}$-bimódulo de Cayley.

Os enunciados e as demonstrações dos Lemas 18 e 19 são análogos a dos Lemas 3.1 e 3.2 de [8], por isso, vamos omitir suas demonstrações.

Lema 18 Seja $V=V_{a} \oplus V_{c}$ um superbimódulo alternativo unitário sobre $\mathbb{O}(4 \mid 4)=\mathbb{H}+M$. Então, para qualquer $v \in V_{c}, m \in M, a \in \mathbb{H}$,

$$
(v m) a=(a v) m, \quad a(m v)=m(v a)
$$

e para quaisquer $u \in V_{a}, m \in M, a, b \in \mathbb{H}$,

$$
\begin{aligned}
(u m) a & =(u \bar{a}) m \\
a(m u) & =m(\bar{a} u), \\
((u m) a) b & =(u m)(b a), \\
b(a(m u)) & =(a b)(m u), \\
(u m, a, b) & =(u m)[b, a], \\
(b, a, m u) & =[b, a](m u),
\end{aligned}
$$

Lema 19 Seja $V=V_{a} \oplus V_{c}$ um superbimódulo alternativo unitário sobre $\mathbb{O}(4 \mid 4)=\mathbb{H}+M$. Então, $V_{a} M, M V_{c}, V_{c} M$ e $M V_{c}$ são subespaços $\mathbb{H}$-invariantes. Além disso, $V_{a} M+M V_{a} \subseteq V_{c}$ e $V_{c} M+M V_{c} \subseteq V_{a}$.

Corolário 42 Nas notações dos Lemas 18 e 19, $V_{a} \neq 0$.

Demonstração: Suponha que $V_{a}=0$, então pelo Lema $19 V M=M V=0$. Escolha $a, b \in \mathbb{H}$, tais que, $\alpha=[a, b]^{2} \neq 0(\alpha \in F)$. Para qualquer $w \in V$, por (4.2)

$$
\begin{aligned}
\alpha \gamma w & =w(\gamma[a, b][\overline{\bar{b}}, \bar{a}]) \\
& =w((v[\bar{b}, \bar{a}])(v[a, b])) \\
& =(w(v[\bar{b}, \bar{a}]))(v[a, b])+((v[\bar{b}, \bar{a}]) w)(v[a, b])-(v[\bar{b}, \bar{a}])(w(v[a, b]))=0 .
\end{aligned}
$$

Como $\alpha, \gamma \neq 0$, temos $w=0$, uma contradição. 
Lema 20 Seja $V=V_{a} \oplus V_{c}$ um superbimódulo alternativo unitário sobre $\mathbb{O}(4 \mid 4)=\mathbb{H}+M$ e $Z_{a}=\left\{v \in V_{a} \mid[v, \mathbb{H}]=0\right\}$. Então, $Z_{a} \neq 0$ e satisfaz as seguintes condições:

i) $\left[Z_{a}, \mathbb{O}(4 \mid 4)\right]=0$,

ii) $\left(Z_{a}, \mathbb{O}(4 \mid 4), \mathbb{O}(4 \mid 4)\right)=0$.

Demonstração: Pelo Corolário $42, V_{a} \neq 0$ e é um $\mathbb{H}$-bimódulo associativo unitário completamente redutível, isto é, $V_{a}=\sum \oplus W_{i}$, onde $W_{i} \simeq \operatorname{Reg} \mathbb{H}$. Assim, $V_{a}$ contem uma família $\left\{u_{i}\right\}$ (as imagens de 1 no isomorfismo com Reg $\mathbb{H}$ ), tais que, $V_{a}=\sum \oplus u_{i} \mathbb{H}$. Agora, é claro que $Z_{a} \neq 0$.

Fixemos elementos arbitrários $u, v, w \in Z_{a}, m, n \in M$ e $a, b, c \in \mathbb{H}$. Vamos mostrar primeiro que

$$
\left(Z_{a}, \mathbb{H}, M\right)=0
$$

Pelos Lemas 18 e 19, temos

$$
(a, u, m)=(a u) m-a(u m)=(a u) m-(u m) \bar{a} \stackrel{(5.5)}{=}(a u) m-(u a) m=[a, u] m=0,
$$

o qual prova (4.11). Além disso, pela superlinearização de (3.21)

$$
([u, m], a, b)=([u, a], m, b)+[m,(u, a, b)]+[a,(u, m, b)]=0 .
$$

Portanto, $\left(\left[Z_{a}, M\right], \mathbb{H}, \mathbb{H}\right)=0$, isto é, $\left[Z_{a}, M\right] \subseteq V_{a}$. Como $\left[Z_{a}, M\right] \subseteq V_{c}$, então

$$
\left[Z_{a}, M\right] \subseteq V_{a} \cap V_{c}=0
$$

o qual, implica $\left[Z_{a}, M\right]=0$. Isto prova $\left.i\right)$.

Pelas superlinearizações de (3.18) e (3.19) e, escolhendo $a, b \in \mathbb{H}$ tais que $[a, b]^{2}=\alpha \in F$, $\alpha \neq 0$, obtemos

$$
\begin{aligned}
{[a, b](u, m, n)=} & {[u, b](a, m, n)+[a, m](u, b, n)+[u, m](a, b, n)+(a, b,(u, m, n)) } \\
& +(u, b,(a, m, n))+(a, m,(u, b, n))+(u, m,(a, b, n)) \\
= & (u, m,(a, b, n)) \\
= & ((u, m, a), b, n)+((u, b, n), m, a)+(u,(m, b, n), a) \\
& +[m,(u, a,[b, n])]+([u, a], m,[b, n])=0 .
\end{aligned}
$$

De forma análoga, $(u, m, n)[a, b]=0$. Logo,

$$
\begin{aligned}
\alpha(u, m, n)=[a, b]^{2}(u, m, n)= & {[a, b]([a, b](u, m, n)) } \\
& +(([a, b](u, m, n))[a, b]-[a, b]((u, m, n)[a, b]))=0
\end{aligned}
$$

e $(u, m, n)=0 ; \operatorname{assim}\left(Z_{a}, M, M\right)=0$. Isto prova $\left.i i\right)$.

Isto prova o Lema.

Q.E.D.

Teorema 43 Cada bimódulo alternativo unitário sobre a superálgebra $\mathbb{O}(4 \mid 4)$ é completamente redutivel. 
Demonstração: Seja $V$ um $\mathbb{O}(4 \mid 4)$-bimódulo alternativo unitário. Então, pela Proposição 16, $V=V_{a} \oplus V_{c}$, onde $V_{a}$ é um $\mathbb{H}$-bimódulo associativo e $V_{c}$ é um $\mathbb{H}$-bimódulo de Cayley. Além disso, o bimódulo $V_{a}$ é isomorfo a uma soma direta de $\mathbb{H}$-bimódulos regulares: $V_{a}=$ $\bigoplus_{i} V_{i}$, onde para cada $i, V_{i}=u_{i} \mathbb{H}$ e $u_{i} \in Z_{a}\left(V_{i}\right)$ é a imagem da identidade 1 no isomorfismo $\varphi: a \mapsto u_{i} a$ dos $\mathbb{H}$-bimódulos Reg $\mathbb{H}$ e $V_{i}$. Em particular, como $1 a=a 1$ para todo $a \in \mathbb{H}$, aplicando $\varphi$ temos $u_{i} a=a u_{i}$; assim $\left[u_{i}, \mathbb{H}\right]=0$. Portanto, $u_{i} \in Z_{a}=Z_{a}(V)=\left\{v \in V_{a}\right.$ : $[v, \mathbb{H}]=0\}$. Pelo Lema 20 , temos $Z_{a} \neq 0$ e

$$
\left(Z_{a}, \mathbb{O}(4 \mid 4), \mathbb{O}(4 \mid 4)\right)=\left[Z_{a}, \mathbb{O}(4 \mid 4)\right]=0 .
$$

Consideremos $W=\sum_{i} u_{i} \mathbb{O}(4 \mid 4)$. É claro que $W$ é um $\mathbb{O}(4 \mid 4)$-subbimódulo de $V$ e

$$
W=\sum_{i} u_{i} \mathbb{O}(4 \mid 4) \supseteq \sum_{i} u_{i} \mathbb{H}=V_{a}
$$

Seja $w \in V_{c}$. Então,

$$
\begin{aligned}
\alpha \beta w & =w(\alpha[a, b][\bar{b}, \bar{a}]) \\
& =w((v[\bar{b}, \bar{a}])(v[a, b])) \\
& =(w(v[\bar{b}, \bar{a}]))(v[a, b])+((v[\bar{b}, \bar{a}]) w)(v[a, b])-(v[\bar{b}, \bar{a}])(w(v[a, b])) .
\end{aligned}
$$

Pelo Lema $19 w(v[\bar{b}, \bar{a}])(v[\bar{b}, \bar{a}]) w, w(v[a, b]) \in V_{a}$; assim $w \in W$. Portanto, $V_{c} \subseteq W$ e $W=V$. Como cada bimódulo $u_{i} \mathbb{O}(4 \mid 4)$ é irredutível; portanto $V=W$ é completamente redutível.

Isto prova o Teorema.

Q.E.D.

Teorema 44 Cada bimódulo alternativo unitário sobre a superálgebra $\mathbb{O}[u]$ é completamente redutivel.

Demonstração: Seja $V$ um $\mathbb{O}[u]$-bimódulo alternativo unitário. Como a álgebra de CayleyDickson $\mathbb{O}$ é uma subálgebra da superálgebra alternativa $\mathbb{O}[u]$, podemos considerar $V$ como um $\mathbb{O}$-bimódulo alternativo unitário. Então, pelo Teorema 35, $V$ é completamente redutível, isto é, $V=\sum \oplus V_{i}$ e existe uma família $\left\{u_{i}\right\}$ de elementos de $V$ (as imagens de 1 no isomorfismo com $\operatorname{Reg}(\mathbb{O})$, tais que, $\left[u_{i}, \mathbb{O}\right]=0$ e $V_{i}=u_{i} \mathbb{O} \simeq \operatorname{Reg} \mathbb{O}$. Além disso, na prova do Teorema 6.1 de [17] tem-se mostrado que $\left(V_{i}, u, \mathbb{O}[u]\right)=\left[V_{i}, u\right]=0$ para cada $i$; assim $\left[u_{i}, u\right]=0$. Então, pela superlinearização de (3.15) e para todo $a \in \mathbb{O}$, temos

$$
\left[u_{i}, a u\right]=\left[u_{i}, a\right] u+a\left[u_{i}, u\right]+3\left(u_{i}, a, u\right)=0
$$

$\operatorname{assim}\left[u_{i}, \mathbb{O} u\right]=0$. Portanto, $\left[u_{i}, \mathbb{O}[u]\right]=\left[u_{i}, \mathbb{O}\right]+\left[u_{i}, \mathbb{O} u\right]=0$.

Também, pela superlinearização de (3.15)

$$
3\left(\mathbb{O}[u], \mathbb{O}[u], u_{i}\right) \subseteq\left[\mathbb{O}[u] \mathbb{O}[u], u_{i}\right]+\mathbb{O}[u]\left[\mathbb{O}[u], u_{i}\right]+\left[\mathbb{O}[u], u_{i}\right] \mathbb{O}[u]=0 ;
$$

portanto $\left(\mathbb{O}[u], \mathbb{O}[u], u_{i}\right)=0$.

Consideremos $W=\sum_{i} u_{i} \mathbb{O}[u]$. É claro que $W$ é um $\mathbb{O}[u]$-subbimódulo de $V$ e

$$
W=\sum_{i} u_{i} \mathbb{O}[u] \supseteq \sum_{i} u_{i} \mathbb{O}=V .
$$

Como cada bimódulo $u_{i} \mathbb{O}[u]$ é irredutível; portanto $V=W$ é completamente reductível.

Isto prova o Teorema. 
No que segue, provamos para as superálgebras alternativas simples $\mathbb{O}(4 \mid 4)$ e $\mathbb{O}[u]$ uns análogos do Teorema de Fatorização de Kronecker.

Teorema 45 Seja $\mathcal{A}$ uma superálgebra alternativa, tal que, $\mathcal{A}$ contem $\mathbb{O}(4 \mid 4)$ com o mesmo elemento identidade. Então, $\mathcal{A} \cong U \otimes_{F} \mathbb{O}(4 \mid 4)$ para uma certa superálgebra associativa $e$ comutativa $U$.

Demonstração: Considere $\mathcal{A}$ como um $\mathbb{O}(4 \mid 4)$-bimódulo alternativo unitário. Pelo Teorema $43, \mathcal{A}=\sum_{i} u_{i} \mathbb{O}(4 \mid 4)$, onde $u_{i} \in Z_{a}=Z_{a}(\mathcal{A})=\{u \in \mathcal{A}:[u, \mathbb{O}(4 \mid 4)]=0\}$ é um elemento homogêneo. Se denotamos $U=Z_{a}$. Então, $\mathcal{A}=U \mathbb{O}(4 \mid 4)$. Além disso, pelo Lema $20, U \neq 0$ e $[U, \mathbb{O}(4 \mid 4)]=(U, \mathbb{O}(4 \mid 4), \mathbb{O}(4 \mid 4))=0$. Mostraremos que $U$ é a desejada superálgebra.

Fixemos elementos arbitrários $u, w \in U, a, b, c \in \mathbb{O}(4 \mid 4)$ e $m \in M=v \mathbb{H}$.

Se $a, b, c \in \mathbb{H}$. Então, pela superlinearização de (3.18) e (3.19), e escolhendo $a, b \in \mathbb{H}$, tais que, $[a, b]^{2}=\alpha \in F, \alpha \neq 0$, temos para qualquer $x \in U$

$$
\begin{aligned}
{[a, b](u, x, c)=} & {[u, b](a, x, c)+[a, x](u, b, c)+[u, x](a, b, c)+(a, b,(u, x, c)) } \\
& +(u, b,(a, x, c))+(a, x,(u, b, c))+(u, x,(a, b, c)) \\
= & (a, b,(u, x, c)) \\
= & ((u, a, b), x, c)+(u,(x, a, b), c)+(u, x,(c, a, b)) \\
& +[x,(u, c,[a, b])]+([u, c], x,[a, b])=0 .
\end{aligned}
$$

De forma análoga, $(u, x, c)[a, b]=0$. Logo,

$$
\begin{aligned}
\alpha(u, x, c) & =[a, b]^{2}(u, x, c) \\
& =[a, b]([a, b](u, x, c))+(([a, b](u, x, c))[a, b]-[a, b]((u, x, c)[a, b]))=0
\end{aligned}
$$

assim, $(u, x, c)=0$; portanto $(U, U, \mathbb{H})=0$. Novamente, pela superlinearização de $(3.18)$ e $(3.19)$

$$
\begin{aligned}
{[a, b](u, n, v)=} & {[u, b](a, n, v)+[a, n](u, b, v)+[u, n](a, b, v)+(a, b,(u, n, v)) } \\
& +(u, b,(a, n, v))+(a, n,(u, b, v))+(u, n,(a, b, v)) \\
= & (a, b,(u, n, v)) \\
= & ((u, n, a), b, v)+((u, b, v), n, a)+(u,(n, b, v), a) \\
& +[n,(u, a,[b, v])]+([u, a], n,[b, v])=0 .
\end{aligned}
$$

Similarmente, $(u, n, v)[a, b]=0$. Assim,

$$
\begin{aligned}
\alpha(u, n, v) & =[a, b]^{2}(u, n, v) \\
& =[a, b]([a, b](u, n, v))+([a, b](u, n, v))[a, b]+[a, b]((u, n, v)[a, b])=0
\end{aligned}
$$

e $(u, n, v)=0 ; \operatorname{logo},(U, M, U)=0$. Portanto, $(U, U, \mathbb{O}(4 \mid 4))=0$.

Além disso, pela superlinearização de (3.15)

$$
[u v, a]=u[v, a]+[u, a] v+3(u, v, a)=0,
$$

temos $U^{2} \subseteq U$. Portanto, $U$ é uma subsuperálgebra de $\mathcal{A}$.

Seja $x=\sum u_{i} a_{i} \in U, a_{i} \in \mathbb{O}(4 \mid 4)$. Então, $a x=x a$ para todo $a \in \mathbb{O}(4 \mid 4)$, isto é,

$$
\sum a u_{i} a_{i}=\sum u_{i} a_{i} a
$$




$$
\sum u_{i} a a_{i}=\sum u_{i} a_{i} a
$$

Portanto, $a a_{i}=a_{i} a$ e $a a_{j}=a_{j} a$. Como $\mathbb{O}(4 \mid 4)$ é central, isso, implica $a_{i}=\lambda_{i} 1$ e $a_{j}=\lambda_{j} 1$, onde $\lambda_{i}$ e $\lambda_{j}$ estão no corpo $F$. Portanto, $U$ é o espaço gerado pelos $u_{i}$. Logo, é claro que $\mathcal{A} \cong U \otimes_{F} \mathbb{O}(4 \mid 4)$.

Escolha $a, b \in \mathbb{H}$, tais que, $[a, b]^{2}=\alpha \in F, \alpha \neq 0$. Pela superlinearização de (3.18), obtemos

$$
\begin{aligned}
{[a, b](u, v, w)=} & {[a, v](u, b, w)+[u, b](a, v, w)+[u, v](a, b, w)+(a, b,(u, v, w)) } \\
& +(a, v,(u, b, w))+(u, b,(a, v, w))+(u, v,(a, b, w))=0
\end{aligned}
$$

Similarmente, $(u, v, w)[a, b]=0$. Então,

$$
\begin{aligned}
\alpha(u, v, w)=[a, b]^{2}(u, v, w)= & {[a, b]([a, b](u, v, w)) } \\
& +(([a, b](u, v, w))[a, b]-[a, b]((u, v, w)[a, b]))=0
\end{aligned}
$$

e $(u, v, w)=0$. Portanto, $U$ é associativo.

Aplicando novamente a superlinearização de (3.18), temos

$$
\begin{aligned}
{[u, v](a, b, c)=} & {[a, v](u, b, c)+[u, b](a, v, c)+[a, b](u, v, c)+(u, v,(a, b, c)) } \\
& +(a, v,(u, b, c))+(u, b,(a, v, c))+(a, b,(u, v, c))=0 .
\end{aligned}
$$

Podemos escrever o produto anterior como $[u, v] \otimes(a, b, c)=0$. Assim, como $\mathbb{O}(4 \mid 4)$ é não associativa, existem $a, b, c \in \mathbb{O}(4 \mid 4)$, tais que, $(a, b, c) \neq 0$. Então, $[u, v]=0$ e $[U, U]=0$; portanto, $U$ é uma superálgebra associativa e comutativa.

Isto prova o Teorema.

Q.E.D.

Teorema 46 Seja $\mathcal{A}$ uma superálgebra alternativa, tal que, $\mathcal{A}$ contém $\mathbb{O}[u]$ com o mesmo elemento identidade. Então, $\mathcal{A} \cong \mathcal{U} \otimes_{F} \mathbb{O}[u]$ para uma certa superálgebra associativa e comutativa $\mathcal{U}$.

Demonstração: Considere $\mathcal{A}$ como um $\mathbb{O}[u]$-bimódulo alternativo unitário. Então, pelo Teorema 44, $\mathcal{A}=\sum_{i} u_{i} \mathbb{O}[u]$, onde $u_{i} \in Z_{a}=\{x \in \mathcal{A}:[x, \mathbb{O}[u]]=0\}$ é um elemento homogêneo. Denotemos $\mathcal{U}=Z_{a}$. Então, $\mathcal{A}=\mathcal{U} \mathbb{O}[u]$, mostraremos que $\mathcal{U}$ é a desejada superálgebra.

Provamos primeiro que $\mathcal{U}$ é uma subsuperálgebra de $\mathcal{A}$. Fixemos elementos arbitrários $x, y, z \in \mathcal{U}$ e $a, b, c \in \mathbb{O}[u]$. Então, $\mathcal{U} \neq 0$ e $[\mathcal{U}, \mathbb{O}[u]]=0$. Então, pela superlinearização de $(3.15)$

$$
3(\mathbb{O}[u], \mathbb{O}[u], \mathcal{U}) \subseteq[\mathbb{O}[u] \mathbb{O}[u], \mathcal{U}]+\mathbb{O}[u][\mathbb{O}[u], \mathcal{U}]+[\mathbb{O}[u], \mathcal{U}] \mathbb{O}[u]=0
$$

portanto $(\mathbb{O}[u], \mathbb{O}[u], \mathcal{U})=0$. Em particular, $(\mathbb{O}, \mathbb{O}, \mathcal{U})=0$. Assim, como na prova do Teorema 44 , obtemos $(\mathbb{O}, \mathcal{U}, \mathcal{U})=0$.

Seja $x=\sum u_{i} a_{i} \in \mathcal{U}, a_{i} \in \mathbb{O}[u]$. Então, $a x=x a$ para todo $a \in \mathbb{O}[u]$, isto é,

$$
\begin{aligned}
\sum a u_{i} a_{i} & =\sum u_{i} a_{i} a, \\
\sum u_{i} a a_{i} & =\sum u_{i} a_{i} a .
\end{aligned}
$$

Portanto, $a a_{i}=a_{i} a$ e $a a_{j}=a_{j} a$. Como $\mathbb{O}[u]$ é central, isso, implica $a_{i}=\lambda_{i} 1$ e $a_{j}=\lambda_{j} 1$, onde $\lambda_{i}$ e $\lambda_{j}$ estão no corpo $F$. Portanto, $U$ é o espaço gerado pelos $u_{i}$. Portanto, é claro que $\mathcal{A} \cong \mathcal{U} \otimes_{F} \mathbb{O}[u]$. 
80

TEOREMAS DE FATORIZAÇÃO DE KRONECKER PARA AS SUPERÁLGEBRAS

ALTERNATIVAS

Pela superlinearização de (3.18) e (3.19), e escolhendo $a, b \in \mathbb{H}$ tais que $[a, b]^{2}=\alpha \in F$, $\alpha \neq 0$ obtemos

$$
\begin{aligned}
{[a, b](\mathcal{U}, u, \mathcal{U}) \subseteq } & {[\mathcal{U}, b](a, u, \mathcal{U})+[a, u](\mathcal{U}, b, \mathcal{U})+[\mathcal{U}, u](a, b, \mathcal{U})+(a, b,(\mathcal{U}, u, \mathcal{U})) } \\
& +(\mathcal{U}, b,(a, u, \mathcal{U}))+(a, u,(\mathcal{U}, b, \mathcal{U}))+(\mathcal{U}, u,(a, b, \mathcal{U})) \\
= & (a, b,(\mathcal{U}, u, \mathcal{U})) \\
\subseteq & ((\mathcal{U}, a, b), u, \mathcal{U})+(\mathcal{U},(u, a, b), \mathcal{U})+(\mathcal{U}, u,(\mathcal{U}, a, b)) \\
& +[u,(\mathcal{U}, \mathcal{U},[a, b])]-([\mathcal{U}, \mathcal{U}], u,[a, b])=0
\end{aligned}
$$

pois $(\mathcal{U}, \mathcal{U},[a, b]) \subseteq \mathcal{A}$ e $[\mathcal{U}, \mathcal{U}] \subseteq \mathcal{A}$. Similarmente, $(\mathcal{U}, u, \mathcal{U})[a, b]=0$. Logo,

$$
\begin{aligned}
\alpha(\mathcal{U}, u, \mathcal{U})=[a, b]^{2}(\mathcal{U}, u, \mathcal{U})= & {[a, b]([a, b](\mathcal{U}, u, \mathcal{U})) } \\
& +(([a, b](\mathcal{U}, u, \mathcal{U}))[a, b]-[a, b]((\mathcal{U}, u, \mathcal{U})[a, b]))=0
\end{aligned}
$$

Consequentemente, $(\mathcal{U}, u, \mathcal{U})=0$. Também, pela superlinearização de (3.15)

$$
\begin{aligned}
{[[x, y], a]=} & {[x y, a]+[y x, a] } \\
= & x[y, a]+[x, a] y+3(x, y, a) \\
& +y[x, a]+[y, a] x+3(y, x, a)=6(x, y, a)=0,
\end{aligned}
$$

isto é, $[[\mathcal{U}, \mathcal{U}], \mathbb{O}[u]]=0$; portanto $[\mathcal{U}, \mathcal{U}] \subseteq \mathcal{U}$. Assim, pela superlinearização de (3.16)

$$
(\mathbb{O} u, \mathcal{U}, \mathcal{U}) \subseteq \mathbb{O}(u, \mathcal{U}, \mathcal{U})+(\mathbb{O}, \mathcal{U}, \mathcal{U}) u+(\mathbb{O}, u,[\mathcal{U}, \mathcal{U}])=0
$$

e $(\mathbb{O} u, \mathcal{U}, \mathcal{U})=0$. Portanto, $(\mathbb{O}[u], \mathcal{U}, \mathcal{U})=(\mathbb{O}, \mathcal{U}, \mathcal{U})+(\mathbb{O} u, \mathcal{U}, \mathcal{U})=0$.

Agora, novamente pela superlinearização de (3.15) temos

$$
[x y, a]=x[y, a]+[x, a] y+3(x, y, a)=0 ;
$$

portanto $\mathcal{U}^{2} \subseteq \mathcal{U}$. Além disso, pela superlinearização de (3.18), obtemos

$$
\begin{aligned}
{[a, b](x, y, z)=} & {[a, y](x, b, z)+[x, b](a, y, z)+[x, y](a, b, z)+(a, b,(x, y, z)) } \\
& +(a, y,(x, b, z))+(x, b,(a, y, z))+(x, y,(a, b, z))=0 .
\end{aligned}
$$

Similarmente, $(x, y, z)[a, b]=0$. Escolha $a, b \in \mathbb{H}$ tais que $[a, b]^{2}=\alpha \in F, \alpha \neq 0$. Então,

$$
\begin{aligned}
\alpha(x, y, z)=[a, b]^{2}(x, y, z)= & {[a, b]([a, b](x, y, z)) } \\
& +(([a, b](x, y, z))[a, b]-[a, b]((x, y, z)[a, b]))=0
\end{aligned}
$$

e $(x, y, z)=0$; portanto, $\mathcal{U}$ é associativa.

Finalmente, pela superlinearização de (3.18), temos

$$
\begin{aligned}
{[x, y](a, b, c)=} & {[a, y](x, b, c)+[x, b](a, y, c)+[a, b](x, y, c)+(x, y,(a, b, c)) } \\
& +(a, y,(x, b, c))+(x, b,(a, y, c))+(a, b,(x, y, c))=0 .
\end{aligned}
$$

Podemos escrever o produto anterior como $[x, y] \otimes(a, b, c)=0$. Assim, como $\mathbb{O}[u]$ é não associativa, existem $a, b, c \in \mathbb{O}[u]$, tais que, $(a, b, c) \neq 0$. Então, $[x, y]=0$ e $[\mathcal{U}, \mathcal{U}]=0$; portanto, $\mathcal{U}$ é uma superálgebra associativa e comutativa.

Isto prova o teorema. 
TEOREMAS DE FATORIZAÇÃO DE KRONECKER 81

Q.E.D. 
82 TEOREMAS DE FATORIZAÇÃO DE KRONECKER PARA AS SUPERÁLGEBRAS ALTERNATIVAS 


\section{Referências Bibliográficas}

[1] N. Jacobson, Structure and Representations of Jordan Algebras. AMS, Providence, RI, 1968. 1, 62, 63

[2] N. Jacobson, A Kronecker factorization theorem for Cayley algebras and the exceptional simple Jordan algebra. Amer. J. Math. 76, (1954). 447-452. 1, 2, 31, 34, 65, 69, 71, 73

[3] N. Jacobson, Structure of alternative and Jordan bimodules. Osaka Math. J. 6, (1954). 1-71. 1, 2, 30, 31, 52, 70

[4] I. Kaplansky, Semi-simple alternative rings, Portugal. Math.10 (1966), 37-50. 1, 65

[5] I. Kashuba, S. Ovsienko, I. Shestakov. Representation type of Jordan algebras. Adv. Math. 226 (2011), no. 1, 385-418. 31, 49, 50

[6] E. N. Kuzmin, I. P. Shestakov, Nonassociative structures, VINITI, Itogi nauki i tekhniki, seria "Fundamental Branches", v.57, 179-266, Moscow, 1990; English transl. in "Encyclopaedia of Math. Sciences, v.57, Algebra VI, 199-280", edited by A.I.Kostrikin and I.R.Shafarevich, Springer-Verlag. 1, 2, 5

[7] López-Díaz, M. C., Shestakov, Ivan P., Representations of exceptional simple Jordan superalgebras of characteristic 3. Comm. Algebra 33 (2005), no. 1, 331-337. 65

[8] López-Diaz, M. C., Shestakov, Ivan P., Representations of exceptional simple alternative superalgebras of characteristic 3. Trans. Amer. Math. Soc. 354 (2002), no. 7, 2745-2758. $36,65,75$

[9] Martinez, C., Zelmanov, E., A Kronecker factorization theorem for the exceptional Jordan superalgebra. Journal of Pure and Applied Algebra 177 (2003) no.1, 71-78. 65

[10] R. S. Pierce, Associative algebras. Springer-Verlag, N.Y., 1982. 36

[11] N.A. Pisarenko, The structure of alternative superbimodules. Algebra and Logic 33 (1995), no. 6, 386-397. 73

[12] R. D. Schafer, An introduction to nonassociative algebras. Academic Press, N.Y., 1966. $1,2,5,26$

[13] R. D. Schafer, Alternative algebras over an arbitrary field. Bull. Amer. Math. Soc. 49 (1943), 549-555. 31

[14] R. D. Schafer, Representations of alternative algebras. Trans. Amer. Math. Soc. 72 (1952), 1-17. 31

[15] I. P. Shestakov, Alternative and Jordan superalgebras. Siberian Adv. Math. 9 (1999), no. $2,83-99.2,5,66,74$ 
84 REFERÊNCIAS BIBLIOGRÁFICAS

[16] I. P. Shestakov, Prime Alternative superalgebras of arbitrary characteristic, Algebra i Logika. 36 (1997), no. 6, 675-716; English transl.: Algebra and Logic 36, no.6 (1997), 389-420. 2, 31, 35, 38, 41, 47, 48, 63, 66, 67

[17] Trushina, M.N., Shestakov, I. P., Irreducible bimodules over alternative algebras and superalgebras. Trans. Amer. Math. Soc. 368 (2016), no. 7, 4657-4684. 65, 75, 77

[18] C. T. C. Wall, Graded Brauer groups. Portugal. J. Reine Angew Math. 213(1964). 2, 67

[19] K. Zhevlakov, I. Shestakov, A. Slin'ko, A.Sंhirshov, Rings that are nearly associative, Academic Press, N.Y., 1982. 1, 2, 5, 6, 11, 15, 31, 32, 33, 38, 55, 62, 66 